Teoria de estabilidade em sistemas semidinâmicos impulsivos

Jaqueline da Costa Ferreira 


\section{Teoria de estabilidade em sistemas semidinâmicos impulsivos}

\section{Jaqueline da Costa Ferreira}

Orientador: Prof. Dr. Everaldo de Mello Bonotto

Dissertação apresentada ao Instituto de Ciências Matemáticas e de Computação - ICMC-USP, como parte dos requisitos para obtenção do título de Mestre em Ciências - Matemática . EXEMPLAR DE DEFESA

USP - São Carlos

Dezembro de 2011 
Ficha catalográfica elaborada pela Biblioteca Prof. Achille Bassi e Seção Técnica de Informática, ICMC/USP, com os dados fornecidos pelo(a) autor(a)

Ferreira, Jaqueline da Costa
Teoria de estabilidade em sistemas semidinâmicos
impulsivos / Jaqueline da Costa Ferreira; orientador
Everaldo de Mello Bonotto. - - São Carlos, 2011.
106 p.
Dissertação (Mestrado - Programa de Pós-Graduação en
Matemática) - Instituto de Ciências Matemáticas e
de Computação, Universidade de São Paulo, 2011.
1. Sistemas semidinâmicos impulsivos. 2. Teoria de
estabilidade. I. Bonotto, Everaldo de Mello, orient.
TI. Titulo.


À minha mãe Zelita e ao meu irmão José Henrique. 



\section{Agradecimentos}

Agradeço primeiramente ao maior de todos os matemáticos, o mais perfeito geometra e analista que já existiu, aquele que integrou as mais belas formas derivando das suas mais perfeitas idéias, a Deus que me ajudou a chegar até aqui, que ele continue me guiando para que eu consiga superar todas as dificuldades e que eu possa ser uma milionésima parte do matemático que ele foi.

Agradeço a minha amada mãe, pelo amor e apoio incondicional, pelos conselhos e preocupações, e que junto com meu irmão formam minha família, base de tudo que sou como pessoa e a qual devo tudo que tenho.

Aos meus amigos, Micheli e José Carlos, que fizeram dos quatro difíceis anos de graduação na UFES um período ímpar da minha vida. A toda minha turma de mestrado do ICMC, a lista é grande, mas a todos agradeço pela hospitalidade em São Carlos e a companhia.

Ao meu orientador, o professor Everaldo de Mello Bonotto, pela disponibilidade integral, paciência, e pelos ensinamentos que possibilitaram a realização desse trabalho.

Aos professores e funcionários da Universidade Federal do Espírito Santo - UFES, e da Universidade de São Paulo - USP.

Agradeço a Fundação de Amparo á Pesquisa do Estado de São Paulo - FAPESP, e a Coordenação de Aperfeiçoamento de Pessoal de Nível Superior - CAPES pelo apoio financeiro. 



\section{Resumo}

A teoria de sistemas semidinâmicos impulsivos é utilizada no estudo de sistemas que descrevem processos de evolução que sofrem variações de estado de curta duração e que podem ser consideradas instantâneas. Este fenômeno é chamado de impulso. Para muitos fenômenos naturais, os modelos determinísticos mais realistas são frequentemente descritos por sistemas que envolvem impulsos. Este trabalho se insere no estudo da teoria de estabilidade em sistemas semidinâmicos com ação impulsiva. 



\section{Abstract}

The theory of impulsive semidynamical systems is used in the analysis of systems which describe the evolution of process whose continuous dynamics are interrupted by abrupt changes of state. This phenomenon is called impulse. In many natural phenomena, the real deterministic models are often described by systems which involve impulses. In this work, we study the stability theory in impulsive semidynamical systems. 



\section{Sumário}

$\begin{array}{ll}\text { Introdução } & 1\end{array}$

$\begin{array}{ll}\text { Notações } & 3\end{array}$

1 Preliminares $\quad 5$

1.1 Equações diferenciais com impulsos . . . . . . . . . . . . . . . 5

1.1.1 Sistemas com impulsos . . . . . . . . . . . . . . 5 5

1.1.2 Sistemas com impulsos em tempos fixados . . . . . . . . . . . 7

1.1.3 Sistemas com impulsos em tempos variáveis . . . . . . . . . . . 10

1.1.4 Sistemas autonômos com impulsos . . . . . . . . . . . . . 13

1.1.5 Existência de soluções ..................... 15

1.2 Sistemas semidinâmicos . . . . . . . . . . . . . . . . 19

1.2.1 Conjuntos limites . . . . . . . . . . . . . . . 21

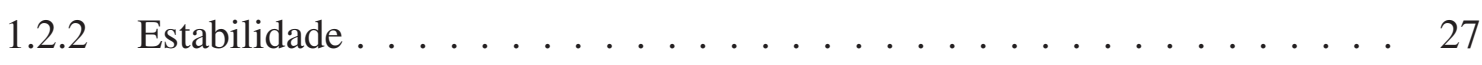

2 Sistemas semidinâmicos impulsivos $\quad 29$

2.1 Descrição de um sistema semidinâmico com impulsos . . . . . . . . . . . . . . 29

2.2 Continuidade da função $\phi \ldots \ldots \ldots \ldots \ldots$ 
2.3 Invariância . . . . . . . . . . . . . . . . . . 40

2.4 Conjuntos limites . . . . . . . . . . . . . . . . . . 41

3 Estabilidade $\quad 47$

3.1 Estabilidade de sistemas semidinâmicos impulsivos . . . . . . . . . . . . . . 47

3.2 Estabilidade de componentes . . . . . . . . . . . . . . . 56

3.3 Estabilidade assintótica . . . . . . . . . . . . . . . . . 59

4 Estabilidade de Lyapunov $\quad 65$

4.1 Critérios de estabilidade . . . . . . . . . . . . . . . . 65

5 Princípio da invariância e teorema da estabilidade assintótica 85

5.1 Princípio da invariância de LaSalle . . . . . . . . . . . . . . . 85

5.2 Teorema da estabilidade assintótica . . . . . . . . . . . . . . 88

6 Atrator Uniforme $\quad 93$

6.1 Região de atração uniforme . . . . . . . . . . . . . . . . . 93

$\begin{array}{ll}\text { Referências Bibliográficas } & 105\end{array}$ 


\section{Introdução}

A teoria de equações diferenciais impulsivas (EDIs) estuda o comportamento de processos de evolução que sofrem variações de estado de curta duração e que podem ser consideradas instantâneas. Este fenômeno é chamado impulso ou ação impulsiva e corresponde às descontinuidades de primeira espécie das soluções ou de suas derivadas. Uma das razões de nosso interesse em EDIs é por elas se constituírem em exemplos de sistemas dinâmicos de dimensão infinita, apresentando dinâmica complexa. A teoria básica de EDIs pode ser encontrada em [19].

Do ponto de vista das aplicações, o interesse das EDIs está em que, para muitos fenômenos naturais, os modelos determinísticos mais realistas são frequentemente descritos por equações que envolvem impulsos. As aplicações das EDIs se dão, especialmente, nas áreas de farmacocinética, tecnologia química, medicina, áreas de controle, finanças, engenharia, física, biologia, entre outras. Esta teoria é uma importante área de investigação que aparece naturalmente na descrição de processos de evolução de vários problemas da realidade.

Uma das aplicações da teoria de EDIs é a teoria de sistemas dinâmicos com impulsos. Sistemas dinâmicos impulsivos é um capítulo importante e moderno da teoria de sistemas dinâmicos topológicos. Esta teoria vem sendo desenvolvida continuamente. Tais sistemas admitem vários fenômenos interessantes às vezes, por causa de sua "irregularidade", e às vezes, por causa da sua "regularidade". Recentemente, vários artigos sobre este tópico foram publicados. Veja, por exemplo [5]-[10] e [13]-[18].

Muitos dos resultados da teoria de sistemas dinâmicos com impulsos são generalizações de resultados da teoria clássica de sistemas dinâmicos contínuos. Além disso, o estudo de sistemas impulsivos leva a novas demonstrações de resultados da teoria de sistemas dinâmicos contínuos.

O objetivo desse trabalho é estudar a teoria de estabilidade para sistemas semidinâmicos com 
impulsos.

Organizamos este trabalho da seguinte forma. No Capítulo 1, apresentamos a teoria de equações diferenciais impulsivas e a teoria de sistemas dinâmicos. Iniciamos com a construção de um sistema diferencial impulsivo e descrevemos alguns tipos de sistemas impulsivos. Na sequência, apresentamos o teorema de existência para EDIs. Finalizamos este capítulo apresentando alguns conceitos importantes de sistemas dinâmicos contínuos para compreensão dos sistemas impulsivos.

No Capítulo 2, apresentamos a definição de um sistema semidinâmico impulsivo. Definimos uma função $\phi$ que representa o menor tempo positivo para o qual a trajetória positiva do sistema encontra o conjunto impulsivo, e fazemos o estudo da continuidade desta função. Na sequência, definimos os conceitos de invariância e conjunto limite para sistemas impulsivos.

No Capítulo 3, estudamos vários tipos de estabilidade para conjuntos. Apresentamos os conceitos de conjuntos estáveis, orbitalmente estáveis, estáveis segundo Bhatia e Hajek, equi estáveis, uniformemente estáveis e assintóticamente estáveis para sistemas impulsivos.

No Capítulo 4, estudamos a estabilidade de conjuntos fechados utilizando funções do tipo Lyapunov. Apresentamos condições necessárias e suficientes para garantir a estabilidade de um conjunto fechado.

No Capítulo 5, apresentamos a versão do princípio da invariância de LaSalle e a versão do Teorema da estabilidade assintótica para sistemas semidinâmicos com impulsos.

Finalizamos este trabalho com o Capítulo 6. Neste capítulo, apresentamos novos resultados sobre a teoria de atrator uniforme para sistemas semidinâmicos com impulsos. Generalizamos o conceito de região de atração uniforme e estabelecemos um resultado de estabilidade assintótica para um conjunto atrator uniforme. 


\section{Notações}

Apresentamos algumas notações que serão utilizadas ao longo do texto.

$\operatorname{Sejam~}(\mathrm{X}, \rho)$ um espaço métrico com métrica $\rho, x \in \mathrm{X}, \varepsilon>0, n$ um natural e $A$ e $B$ subconjuntos não vazios de $X$.

- $\mathbb{R}$ representa o conjunto dos números reais;

- $\mathbb{R}_{+}$representa o conjunto dos números reais não negativos;

- $\mathbb{N}$ representa o conjunto dos números naturais;

- $\mathbb{N}^{*}$ representa o conjunto dos números naturais estritamente positivos;

- $\mathbb{R}^{n}$ representa o espaço euclidiano $n$-dimensional;

- $\bar{A}$ representa o fecho de $A$ em X;

- $\partial A$ representa a fronteira de $A$ em $X$;

- $\rho(x, A)=\inf \{\rho(x, y): y \in A\}$;

- $B(x, \varepsilon)=\{y \in \mathrm{X}: \rho(y, x)<\varepsilon\}$;

- $B(A, \varepsilon)=\{x \in \mathrm{X}: \rho(x, A)<\varepsilon\}$

- $\mathscr{A}(A, \delta, \eta)=\left\{x \in \mathrm{X}: \delta<\rho_{A}(x)<\eta\right\}$

- $C(A, B)$ representa o conjunto das funções contínuas definidas em $A$ com valores em $B$. 
Vamos representar por $\left\{x_{n}\right\}_{n \geq 1}$ uma seqüência em $\mathrm{X}$, onde $n$ pertence ao conjunto dos números naturais $\mathbb{N}^{*}$. Às vezes, representaremos $\lim _{n \rightarrow+\infty} x_{n}=x$ simplesmente como

$$
x_{n} \rightarrow x \quad \text { quando } \quad n \rightarrow+\infty
$$

ou $x_{n} \stackrel{n \rightarrow+\infty}{\longrightarrow} x$.

Quando um conceito é introduzido pela primeira vez, as palavras que o definem estão em negrito. Indicamos o final de uma demonstração com o símbolo $\square$. 


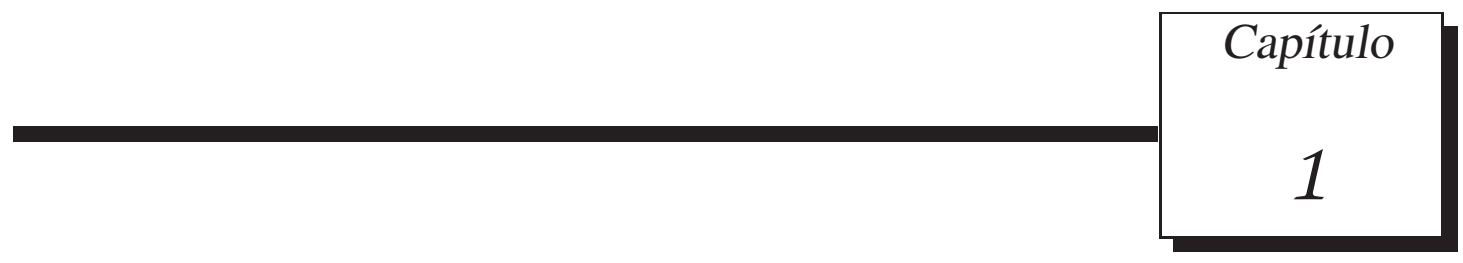

\section{Preliminares}

Neste capítulo, apresentamos alguns resultados e conceitos básicos da teoria de equações diferenciais impulsivas e de sistemas semidinâmicos.

\subsection{Equações diferenciais com impulsos}

As principais referências para esta seção são [3] e [19].

\subsubsection{Sistemas com impulsos}

Sejam $\Omega$ um subconjunto aberto de $\mathbb{R}^{n}$ e $f: \mathbb{R}_{+} \times \Omega \rightarrow \mathbb{R}^{n}$ uma função contínua. Um sistema impulsivo é constituído por:

i) uma equação diferencial

$$
\dot{x}=f(t, x) \quad\left(\dot{x}=\frac{d x}{d t}\right) ;
$$

ii) subconjuntos $M(t), N(t) \subset \Omega, t \in \mathbb{R}_{+}$;

iii) funções $A(t): M(t) \rightarrow N(t), t \in \mathbb{R}_{+}$. 
O processo de evolução do sistema $i$ ), $i i), i i i)$, ocorre da seguinte maneira: consideramos uma solução $x(t)=x\left(t ; t_{0}, x_{0}\right)$ do sistema (1.1) passando pelo ponto $\left(t_{0}, x_{0}\right) \in \mathbb{R}_{+} \times \Omega$. O ponto $P_{t}=(t, x(t))$ inicia seu movimento no ponto $P_{t_{0}}=\left(t_{0}, x_{0}\right)$ e move-se ao longo da curva $\left\{(t, x): t \geq t_{0} \quad\right.$ e $\left.\quad x=x(t)\right\}$ até o tempo $t_{1}>t_{0}$ no qual $P_{t}$ encontra o par $(t, M(t))$. No instante $t=t_{1}$, o operador $A\left(t_{1}\right)$ transfere o ponto $x\left(t_{1}\right)$ para o ponto $x_{1}^{+}=A\left(t_{1}\right) x\left(t_{1}\right) \in N\left(t_{1}\right)$ e assim, o ponto $P_{t_{1}}$ é transferido para o ponto $P_{t_{1}^{+}}=\left(t_{1}, x_{1}^{+}\right) \in\left(t_{1}, N\left(t_{1}\right)\right)$. Deste modo, o ponto $P_{t}$ continua seu movimento ao longo da curva $x(t)=x\left(t ; t_{1}, x_{1}^{+}\right)$, que é solução de (1.1) com condição inicial $P_{t_{1}^{+}}=\left(t_{1}, x_{1}^{+}\right)$, até encontrar novamente o conjunto $(t, M(t))$ em um tempo $t_{2}>t_{1}$. Em seguida, o ponto $x\left(t_{2}\right)$ é transferido pelo operador $A\left(t_{2}\right)$ para o ponto $x_{2}^{+}=A\left(t_{2}\right) x\left(t_{2}\right) \in N\left(t_{2}\right)$. Assim, o ponto $P_{t_{2}}=\left(t_{2}, x\left(t_{2}\right)\right)$ é transferido para o ponto $P_{t_{2}^{+}}=\left(t_{2}, x_{2}^{+}\right) \in\left(t_{2}, N\left(t_{2}\right)\right)$. Agora, o movimento de $P_{t}$ se inicia em $\left(t_{2}, x_{2}^{+}\right)$ao longo da solução $x(t)=x\left(t ; t_{2}, x_{2}^{+}\right)$de $(1.1)$. O processo segue, indutivamente, ao longo da solução de (1.1), caso esta exista. A Figura 1.1 apresenta a evolução de um sistema impulsivo.

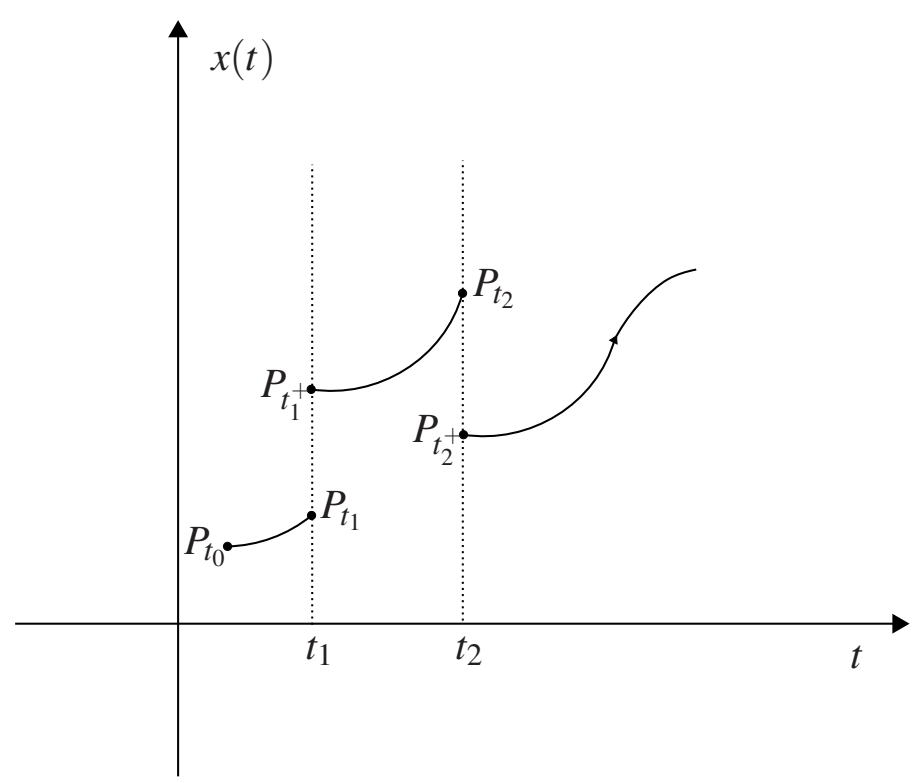

Figura 1.1: Evolução de um sistema impulsivo.

A curva que descreve o movimento de $P_{t}$ acima é denominada curva integral e a função que define esta curva é a solução do sistema diferencial impulsivo.

Uma solução de um sistema diferencial impulsivo pode ser:

a) uma função contínua, se ela não interceptar o conjunto $M(t)$ ou se ela atingir $M(t)$ somente nos pontos fixos do operador $A(t)$; 
b) uma função contínua por partes com um número finito de descontinuidades de primeira espécie, se ela encontrar o conjunto $M(t)$ em um número finito de pontos que não são pontos fixos do operador $A(t)$;

c) uma função contínua por partes com uma quantidade enumerável de descontinuidades de primeira espécie, se ela encontrar o conjunto $M(t)$ em uma quantidade enumerável de pontos que não são pontos fixos do operador $A(t)$.

Os instantes $t=t_{k}$ nos quais a curva integral atinge $M(t)$ são chamados de momentos de impulso das soluções do sistema impulsivo. Assumiremos que a solução $x(t)$ do sistema impulsivo seja contínua à direita em $t_{k}$ e que o limite à esquerda em $t_{k}$ exista, para cada $k=1,2,3, \ldots$, isto é, $x\left(t_{k}^{+}\right):=\lim _{h \rightarrow 0^{+}} x\left(t_{k}+h\right)=x\left(t_{k}\right)$ e $x\left(t_{k}^{-}\right):=\lim _{h \rightarrow 0^{+}} x\left(t_{k}-h\right)<\infty$.

Chamamos um sistema diferencial impulsivo de equação diferencial impulsiva (EDI).

Vamos analisar, a seguir, alguns tipos de sistemas diferenciais com impulsos.

\subsubsection{Sistemas com impulsos em tempos fixados}

Seja $\left\{t_{k}\right\}_{k \geq 1} \subset \mathbb{R}_{+}$uma sequência de instantes tal que $t_{k} \stackrel{k \rightarrow+\infty}{\longrightarrow}+\infty$. Representemos o conjunto $M(t)$ pela sequência de planos $t=t_{k}$ e definamos o operador $A(t)$ no instante $t=t_{k}$ por

$$
\begin{aligned}
A\left(t_{k}\right): \Omega & \rightarrow \Omega \\
x & \mapsto x+I_{k}(x),
\end{aligned}
$$

onde $I_{k}: \Omega \rightarrow \Omega$ é uma função e $k \in\{1,2, \ldots\}$. O conjunto $N(t)$ é definido para $t=t_{k}$ como sendo $N\left(t_{k}\right)=A\left(t_{k}\right) M\left(t_{k}\right)$.

Com a escolha de $M\left(t_{k}\right), N\left(t_{k}\right)$ e $A\left(t_{k}\right)$, obtemos um modelo matemático que descreve um sistema diferencial com impulsos em tempos fixados representado pelo sistema

$$
\left\{\begin{array}{l}
\dot{x}=f(t, x), \quad t \neq t_{k}, \\
\Delta x=I_{k}(x), \quad t=t_{k}, \quad k=1,2, \ldots,
\end{array}\right.
$$


onde, para cada $t=t_{k}$, temos $\Delta x\left(t_{k}\right)=x\left(t_{k}\right)-x\left(t_{k}^{-}\right)$e $x\left(t_{k}^{-}\right)=\lim _{h \rightarrow 0^{+}} x\left(t_{k}-h\right)$. Então qualquer solução $x(t)$ do sistema (1.2) satisfaz as seguintes condições:

i) $\dot{x}(t)=f(t, x(t))$, para $t \in\left[t_{k}, t_{k+1}\right)$;

ii) $\Delta x\left(t_{k}\right)=I_{k}\left(x\left(t_{k}\right)\right)$, para $t=t_{k} \operatorname{com} k=1,2, \ldots$

O comportamento das soluções do sistema (1.2) é influenciado pelos efeitos impulsivos. A seguir, apresentamos dois exemplos que mostram que a continuidade da solução é afetada pela ação impulsiva.

Exemplo 1.1.1 Consideremos a equação diferencial impulsiva

$$
\begin{cases}\dot{x}=0, & t \neq k, \\ \Delta x=\frac{1}{x-1}, & t=k, \quad k=1,2, \ldots\end{cases}
$$

Qualquer solução $x(t)$ da equação diferencial ordinária $\dot{x}=0$, sem efeito impulsivo, é contínua para todo $t \in \mathbb{R}$. No entanto, a solução do sistema (1.3), com condição inicial $x(0)=1$, está definida somente para $0 \leq t \leq 1$, já que a função $I_{k}(x)=\frac{1}{x-1}$ não está definida para $x=1$. A Figura 1.2 mostra a curva integral do sistema com impulsos.

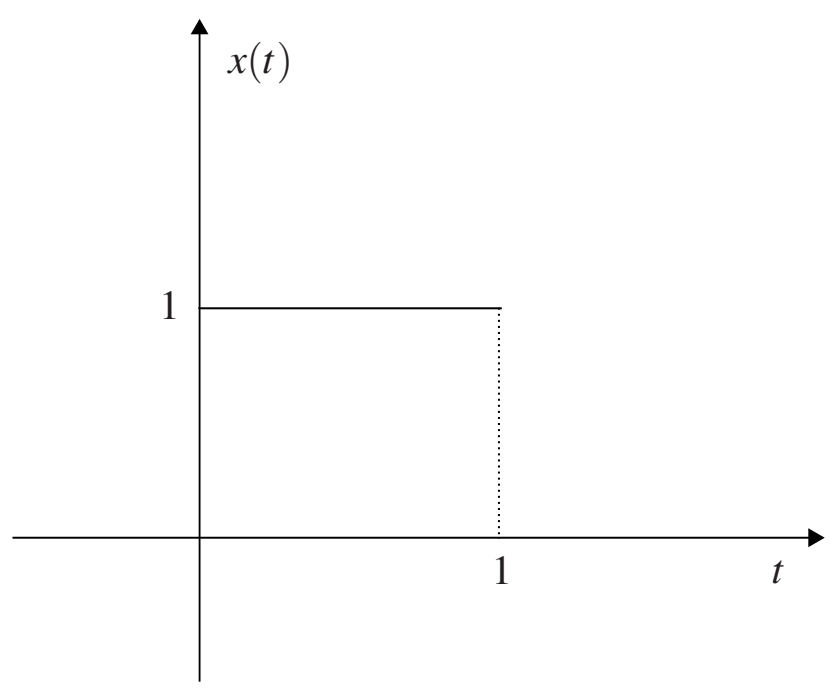

Figura 1.2: Evolução do sistema impulsivo (1.3), com condição inicial $x(0)=1$. 
Exemplo 1.1.2 Consideremos a equação diferencial impulsiva

$$
\begin{cases}\dot{x}=1+x^{2}, & t \neq \frac{k \pi}{4}, \\ \Delta x=-1, & t=\frac{k \pi}{4}, \quad k=1,2, \ldots\end{cases}
$$

Neste caso, a solução $x(t)$ do problema de valor inicial

$$
\left\{\begin{array}{l}
\dot{x}=1+x^{2} \\
x(0)=0
\end{array}\right.
$$

é dada por $x(t)=\operatorname{tg}(t)$, o qual é contínua no intervalo $\left[0, \frac{\pi}{2}\right)$. Por outro lado, a solução do sistema impulsivo (1.4) com condição inicial $x(0)=0$ é dada por

$$
x(t)=\operatorname{tg}\left(t-\frac{k \pi}{4}\right)
$$

para $t \in\left[\frac{k \pi}{4}, \frac{(k+1) \pi}{4}\right)$ e $k=0,1,2, \ldots$. Notemos que a solução é periódica de período $\frac{\pi}{4}$ e tem descontinuidades de primeira espécie nos momentos $t=\frac{k \pi}{4}, k=1,2 \ldots$ A Figura 1.3 ilustra a solução do sistema impulsivo (1.4).

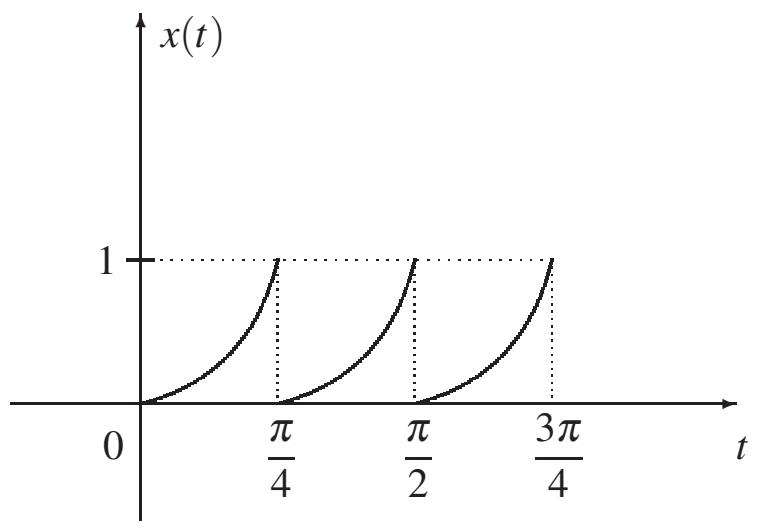

Figura 1.3: Curva integral do sistema impulsivo (1.4), com condição inicial $x(0)=0$. 


\subsubsection{Sistemas com impulsos em tempos variáveis}

Seja $\left\{S_{k}\right\}_{k \geq 0}$ uma sequência de superfícies dada por $S_{k}: t=\tau_{k}(x), k=0,1,2, \ldots$, onde $\tau_{k}: \mathbb{R}^{n} \rightarrow \mathbb{R}$ é uma função que satisfaz as seguintes condições:

i) $\tau_{k}(x)<\tau_{k+1}(x)$, para todo $x \in \mathbb{R}^{n}$ e $k=0,1,2, \ldots$;

ii) $\lim _{k \rightarrow+\infty} \tau_{k}(x)=+\infty$, para todo $x \in \mathbb{R}^{n}$.

Então o sistema diferencial impulsivo

$$
\left\{\begin{array}{l}
\dot{x}=f(t, x), \quad t \neq \tau_{k}(x), \\
\Delta x=I_{k}(x), \quad t=\tau_{k}(x), \quad k=0,1,2, \ldots
\end{array}\right.
$$

descreve um sistema em que os impulsos ocorrem em tempos variáveis.

Os sistemas com impulsos em momentos variáveis apresentam situações que não ocorrem nos sistemas com momentos de impulsos fixados. Por exemplo, notemos que o momento do efeito impulsivo para o sistema (1.5) depende das soluções, isto é, $t_{k}=\tau_{k}\left(x\left(t_{k}\right)\right)$, para cada $k=0,1,2, \ldots$ Assim, soluções iniciadas em diferentes pontos terão diferentes pontos de descontinuidade. Algumas outras situações peculiares também podem ocorrer, por exemplo, uma solução pode atingir a mesma superfície $t=\tau_{k}(x)$ várias vezes e este comportamento é chamado de fenômeno pulso. Notemos também, que duas soluções distintas podem coincidir após algum tempo e se comportar como uma única solução após isso, neste caso chamamos este fenômeno de confluência.

O Exemplo 1.1.3 descreve várias situações que ocorrem com as soluções de um sistema com impulsos em tempo variável.

Exemplo 1.1.3 Consideremos a equação diferencial impulsiva

$$
\left\{\begin{array}{l}
\dot{x}(t)=0, t \neq \tau_{k}(x), \quad t \geq 0, \\
\Delta x=x^{2} \operatorname{sgn} x-x, \quad t=\tau_{k}(x), \quad k=0,1,2, \ldots
\end{array}\right.
$$


onde $\tau_{k}(x)=x+6 k$, com $|x|<3$, descreve a superfície $S_{k}: t=\tau_{k}(x)$ e

$$
\operatorname{sgn}(x)= \begin{cases}1, & x>0 \\ 0, & x=0 \\ -1, & x<0 .\end{cases}
$$

A solução do sistema (1.6) com condição inicial $x(0)=x_{0},\left|x_{0}\right| \geq 3$, é contínua para todo $t \geq 0$, pois a solução não encontra a superfície de impulso. As soluções que se iniciam nos pontos $\left(0, x_{0}\right)$, $1<x_{0}<3$, sofrem efeito impulsivo um número finito de vezes. Por exemplo, a solução $x(t)$ do sistema (1.6) satisfazendo a condição $x(0)=\sqrt[4]{2}$ é dada por

$$
x(t)= \begin{cases}\sqrt[4]{2}, & \text { se } 0 \leq t<\sqrt[4]{2} \\ \sqrt{2}, & \text { se } \sqrt[4]{2} \leq t<\sqrt{2} \\ 2, & \text { se } \sqrt{2} \leq t<2 \\ 4, & \text { se } t \geq 2\end{cases}
$$

Esta solução encontra a superfície $S_{0}$ três vezes e não se choca com qualquer superfície $S_{k}$ além do tempo $t=2$, veja a Figura 1.4 .

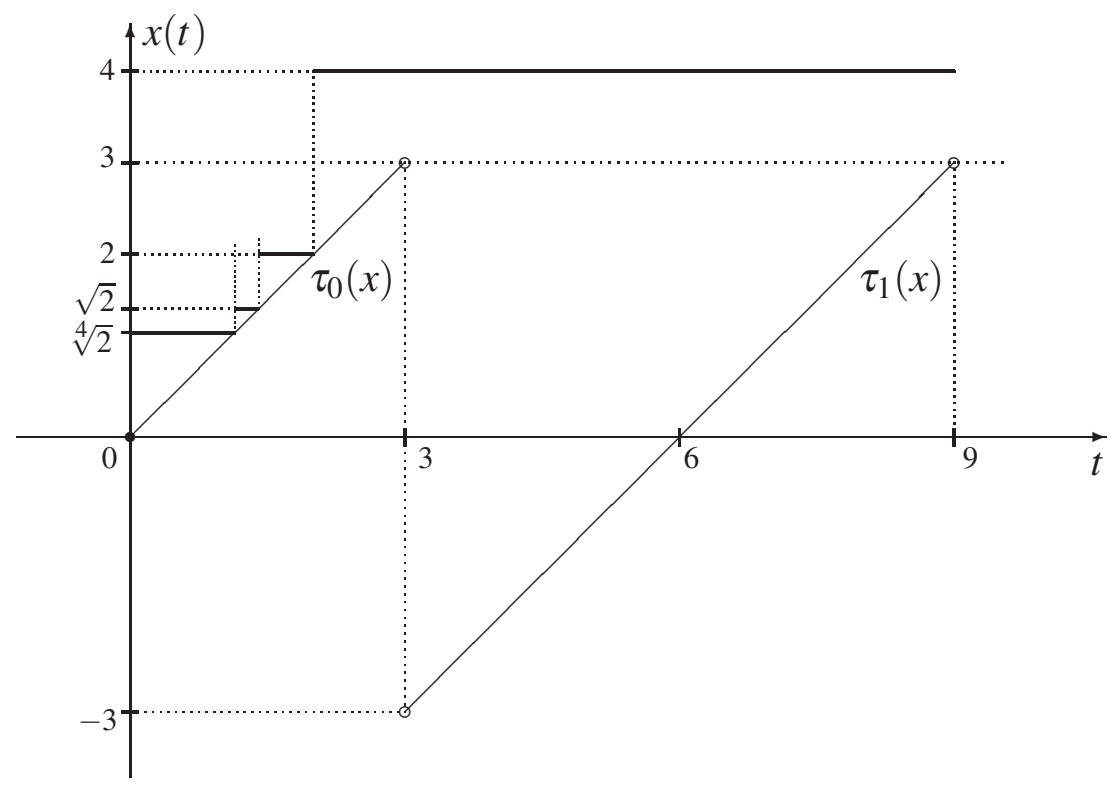

Figura 1.4: Curva integral do sistema impulsivo (1.6) $\operatorname{com} x(0)=\sqrt[4]{2}$. 
Se o ponto inicial $x(0)=x_{0}$ for tal que $0<x_{0}<1$, então a solução $x(t)$ encontrará a superfície $S_{k}$ em um número infinito de tempos $t_{k}$ e teremos $t_{k} \stackrel{k \rightarrow+\infty}{\longrightarrow}+\infty$ bem como $\lim _{k \rightarrow+\infty} x\left(t_{k}\right)=0$. Observemos a Figura 1.5 para $x_{0}=1 / 2$.

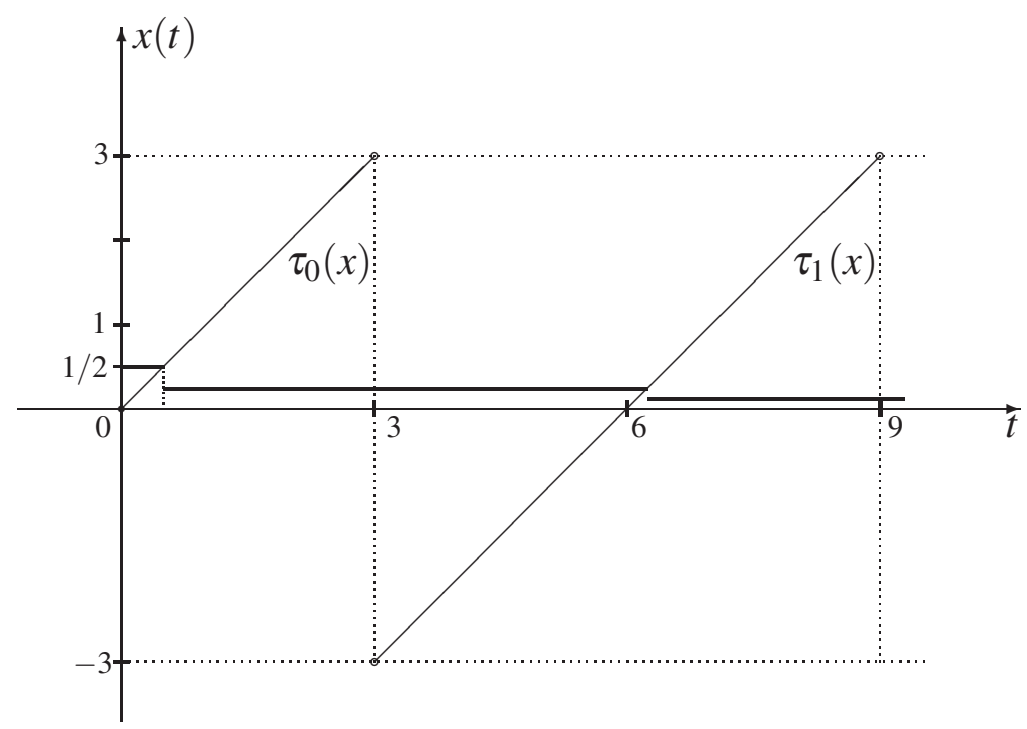

Figura 1.5: Curva integral para o sistema impulsivo (1.6) com $x(0)=1 / 2$.

Quando $-1<x_{0}<0$, a solução $x(t)$ encontrará $S_{k}$ em um número infinito de tempos $t_{k}$ mas, neste caso, temos $\lim _{k \rightarrow+\infty} t_{k}=6$ e $\lim _{k \rightarrow+\infty} x\left(t_{k}\right)=0$. Neste caso a solução apresenta o fenômeno pulso. Veja a Figura 1.6.

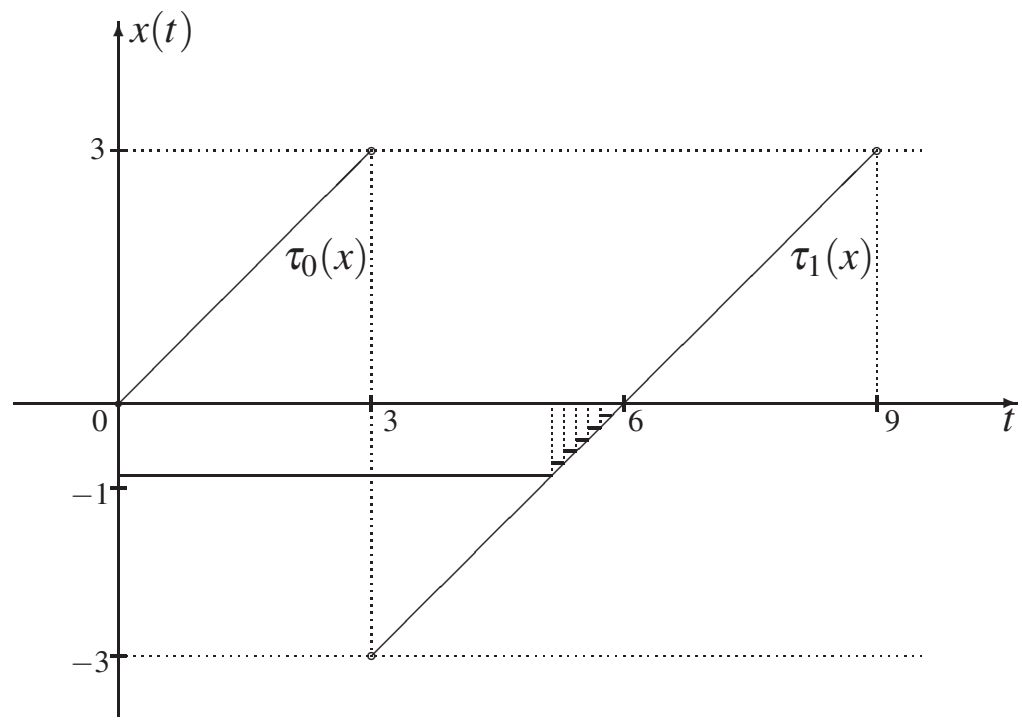

Figura 1.6: Curva integral do sistema impulsivo (1.6) para $-1<x(0)<0$. 
Agora, notemos que as soluções que iniciam nos pontos $(0,0),(0,1)$ e $(0,-1)$ atingem a superfície $S_{k}$ em tempos $t_{k}$ que são pontos fixos do operador $A(t)=x^{2} \operatorname{sgn}(x)$ e, consequentemente, não há efeito impulsivo. Finalmente, as soluções que começam em $(0, \sqrt[4]{2})$ e $(0,4)$ se unem em $t \geq 2$ e, portanto, exibem o fenômeno de confluência.

\subsubsection{Sistemas autonômos com impulsos}

Consideremos os conjuntos $M(t)=M$ e $N(t)=N$ independentes do tempo. Definamos o operador $A(t)=A$ pela lei

$$
\begin{aligned}
A: M & \rightarrow N \\
x & \mapsto x+I(x)
\end{aligned}
$$

onde $I$ é uma função definida em $\Omega$ com valores em $\Omega$ e $\Omega$ é um subconjunto aberto de $\mathbb{R}^{n}$. Assim, obtemos o seguinte sistema diferencial impulsivo autônomo

$$
\left\{\begin{array}{cc}
\dot{x}=f(x), & x \notin M, \\
\Delta x=I(x), & x \in M .
\end{array}\right.
$$

Quando qualquer solução $x(t)=x\left(t ; t_{0}, x_{0}\right)$ do sistema (1.7) encontra $M$ em algum instante $t, \mathrm{o}$ operador $A$ transferirá, instantaneamente, o ponto $x(t) \in M$ para o ponto $y(t)=x(t)+I(x(t)) \in N$. Como (1.7) é autônomo, o movimento do ponto $x(t)$ será considerado em $\Omega$ ao longo da trajetória do sistema (1.7).

A seguir apresentamos um exemplo de um sistema impulsivo autônomo.

Exemplo 1.1.4 Consideremos o sistema diferencial impulsivo em $\mathbb{R}^{2}$ dado por

$$
\left\{\begin{array}{l}
\dot{x}_{1}=x_{1}, \dot{x}_{2}=-\alpha x_{2}, \quad x \notin M, \\
A: M \rightarrow N
\end{array}\right.
$$

onde $0<\alpha<1$ e os conjuntos $M, N \subset \mathbb{R}^{2}$ são definidos por

$$
M=\left\{\left(x_{1}, x_{2}\right) \in \mathbb{R}^{2}: 5 x_{1}^{2}+x_{2}^{2}=8\right\} \text { e } N=\left\{\left(x_{1}, x_{2}\right) \in \mathbb{R}^{2}: x_{1}^{2}+x_{2}^{2}=4\right\} .
$$


O operador $A$ é definido da seguinte forma: dado $x \in M$, consideremos o segmento de reta que liga a origem de $\mathbb{R}^{2}$ ao ponto $x$. O ponto $A(x)$ é definido como sendo o ponto $y \in N$ que pertence a interseção do segmento de reta com o conjunto $N$.

Analisaremos o sistema através das trajetórias $x_{2}=c\left|x_{1}\right|^{\alpha}$, onde $c$ é uma constante.

Primeiramente, notemos que as soluções que iniciam na região $5 x_{1}^{2}+x_{2}^{2}<8$ não sofrem impulsos e tendem à origem. Por outro lado, as soluções que iniciam na região $5 x_{1}^{2}+x_{2}^{2}>8$ sofrem impulsos. Pela simetria do problema, vamos analisar a região $5 x_{1}^{2}+x_{2}^{2}>8 \operatorname{com} x_{1}>0$ e $x_{2}>0$.

Qualquer solução que inicia na região $5 x_{1}^{2}+x_{2}^{2}>8$, com $0 \leq x_{1} \leq 1$, encontra o conjunto $M$ uma única vez. E esta solução é transferida para a região $5 x_{1}^{2}+x_{2}^{2}<8$ tendendo à origem. A Figura 1.7. ilustra este fato.

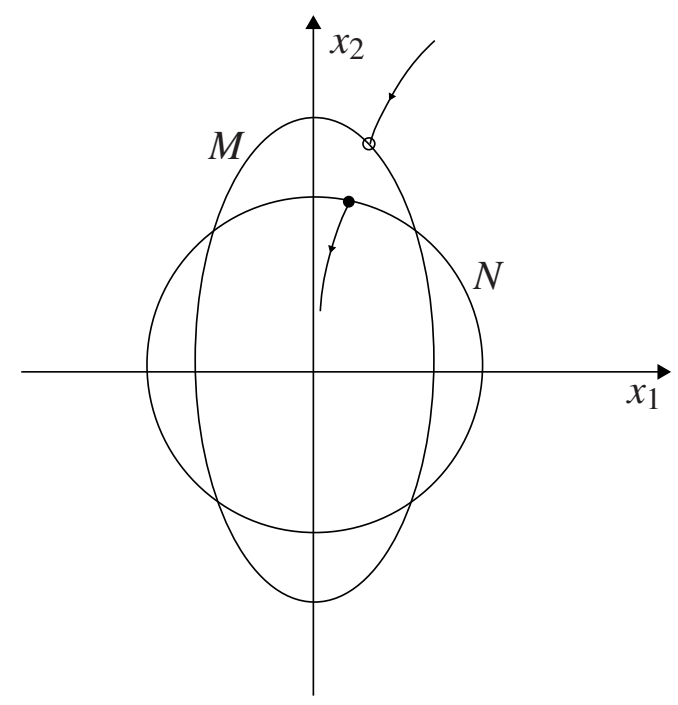

Figura 1.7: Curva integral do sistema impulsivo para $0 \leq x_{1} \leq 1$.

Se a solução inicia seu movimento sobre a curva $x_{2}=\sqrt{3} x_{1}^{\alpha}\left(\operatorname{logo} x_{1} \geq 1\right.$ pois $\left.5 x_{1}^{2}+x_{2}^{2}>8\right)$, então ela encontra $M$ no ponto $(1, \sqrt{3})$ que é um ponto fixo do operador $A$, e portanto a solução não sofre impulso e tende à origem. Veja a Figura 1.8.

Se a solução do sistema encontar $M$ para $1<x_{1}<\sqrt{\frac{8}{5}}$, então ela admitirá infinitos instantes de impulsos pois para $\alpha<1$ temos $x_{1}(t)=e^{-\left(t-t_{0}\right)}<e^{-\alpha\left(t-t_{0}\right)}=x_{2}(t)$ para $t \geq t_{0}$. E a solução tende para o ponto $(1, \sqrt{3})$. Veja a Figura 1.9 .

E finalmemte, se a solução começar na região $x_{1}>1$ e $x_{2}=0$, então ela será um movimento 


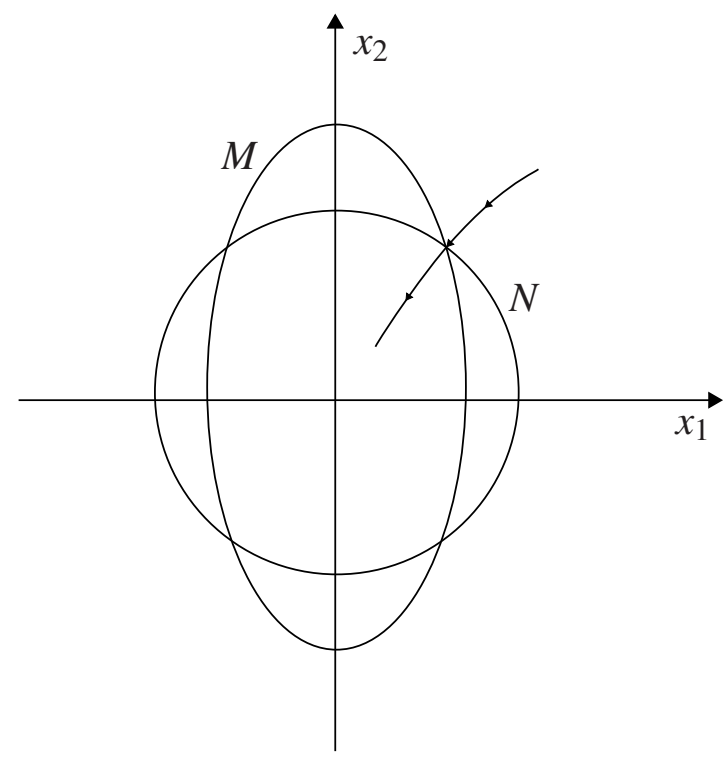

Figura 1.8

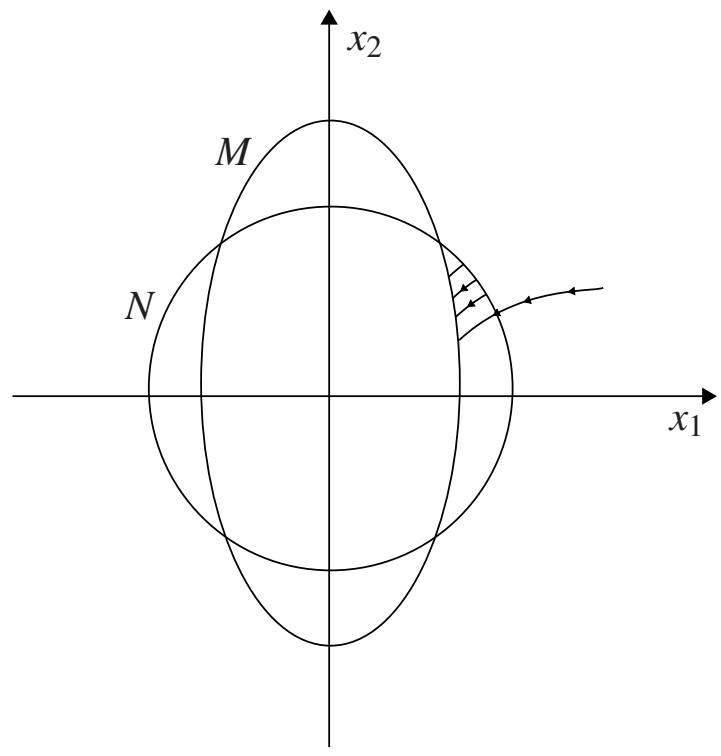

Figura 1.9

periódico de período $\frac{1}{2} \ln \frac{5}{2}$. Neste caso, o operador $A$ transfere o ponto $\left(\sqrt{\frac{8}{5}}, 0\right)$ para o ponto $(2,0)$.

\subsubsection{Existência de soluções}

Sejam $\Omega$ um subconjunto aberto de $\mathbb{R}^{n}$ e $D=\mathbb{R}_{+} \times \Omega$. Dadas as funções $f: D \rightarrow \mathbb{R}^{n}$ e $I_{k}: \Omega \rightarrow \mathbb{R}^{n}, k=1,2,3 \ldots$, consideremos o seguinte sistema diferencial impulsivo:

$$
\left\{\begin{array}{l}
\dot{x}=f(x, t), \quad t \neq \tau_{k}(x), \\
\Delta x=I_{k}(x), \quad t=\tau_{k}(x), \\
x\left(t_{0}^{+}\right)=x_{0}, \quad t_{0} \geq 0 .
\end{array}\right.
$$

Vamos admitir que as seguintes condições sejam válidas para as superfícies de impulsos $\tau_{k}$ :

C1) $\tau_{k} \in C(\Omega,(0,+\infty)), k=1,2,3 \ldots$;

C2) $\tau_{k}(x)<\tau_{k+1}(x)$, para todo $x \in \Omega$ e $k=1,2,3 \ldots$;

C3) $\lim _{k \rightarrow+\infty} \tau_{k}(x)=+\infty$, para todo $x \in \Omega$. 
A seguir definimos o conceito de solução para o sistema (1.8).

Definição 1.1.5 Uma função $x:\left[t_{0}, t_{0}+a\right) \rightarrow \mathbb{R}^{n}, t_{0} \geq 0, a>0$, é chamada solução do sistema (1.8) se as seguintes condições forem válidas:

i) $x\left(t_{0}^{+}\right)=x_{0} \mathrm{e}(t, x(t)) \in D$, para todo $t \in\left[t_{0}, t_{0}+a\right)$;

ii) $x(t)$ é continuamente diferenciável e satisfaz $\dot{x}(t)=f(x(t), t)$ para todo $t \in\left[t_{0}, t_{0}+a\right) \operatorname{com} t \neq \tau_{k}(x(t)) ;$

iii) $x(t)$ é contínua à direita para cada $t \in\left[t_{0}, t_{0}+a\right)$;

$i v)$ se $t \in\left[t_{0}, t_{0}+a\right)$ e $t=\tau_{k}(x(t))$, então $x(t)=x\left(t^{-}\right)+I_{k}(x(t))$ e existe $\delta>0$ tal que $s \neq \tau_{j}(x(s))$ para todo $j \in\{1,2 \ldots\}$ e $t<s<t+\delta$.

Observação 1.1.6 Na definição anterior, item $i i)$, se $t$ é o ponto de extremo $t_{0}$, a derivada $\dot{x}(t)$ é a derivada lateral correspondente.

Diferentemente dos sistemas diferenciais ordinários (EDOs), o sistema (1.8) pode não ter solução, mesmo que $f$ seja contínua ou continuamente diferenciável, já que a solução $x(t)$ do problema $\dot{x}=f(t, x), x\left(t_{0}\right)=x_{0}$, pode estar na superfície $S_{k}$. O exemplo seguinte ilustra este fato.

Exemplo 1.1.7 Consideremos o sistema impulsivo

$$
\begin{cases}\dot{x}=1, & t \neq \tau_{k}(x), \quad k=1,2,3 \ldots \\ \Delta x=x^{2} \operatorname{sgn} x-x, & t=\tau_{k}(x) \\ x(1)=1, & \end{cases}
$$

onde $\tau_{k}(x)=x+6(k-1)$ para $|x|<3, k=1,2, \ldots$. O sistema sem impulsos

$$
\left\{\begin{array}{l}
\dot{x}=1 \\
x(1)=1
\end{array}\right.
$$


admite solução dada por $x(t)=t$. Apesar de $f(x, t)=1$ ser contínua, o sistema (1.9) não admite solução satisfazendo a condição $x(1)=1$. Com efeito, como a solução $x(t)$ de (1.9) se inicia em $t=1 \in \tau_{1}(x)$, temos

$$
\Delta x(1)=1^{2} \operatorname{sgn}(1)-1=0,
$$

ou seja, ela não sai da superfície $\tau_{1}(x)$, o que contradiz a condição $i v$ ) da Definição 1.1.5. Portanto o sistema (1.9) não possui solução.

O Exemplo 1.1.7 mostra que precisamos de algumas condições adicionais sobre $\tau_{k}$ e/ou $f$, além de continuidade, para estabelecermos uma teoria geral de existência para o sistema (1.8). O resultado seguinte, apresenta condições de existência de solução para uma equação diferencial impulsiva.

Teorema 1.1.8 Suponhamos que:

i) $f: D \rightarrow \mathbb{R}^{n}$ seja contínua;

ii) se existir $(\bar{t}, \bar{x}) \in D$ tal que $\bar{t}=\tau_{k}(\bar{x})$ para algum $k \in \mathbb{N}^{*}$, então existirá um $\delta>0$ tal que

$$
t \neq \tau_{k}(x)
$$

para quaisquer $(t, x) \in D$ tais que $0<t-\bar{t}<\delta$ e $|x-\bar{x}|<\delta$.

Então, para cada $\left(t_{0}, x_{0}\right) \in D$ existe uma solução $x:\left[t_{0}, t_{0}+a\right) \rightarrow \mathbb{R}^{n}$ de (1.8) para algum $a>0$.

Demonstração: Pela teoria clássica de equações diferenciais a conclusão é verdadeira para $t_{0} \neq \tau_{k}\left(x_{0}\right)$ qualquer que seja $k \in\{1,2, \ldots\}$.

Suponhamos, então, que $t_{0}=\tau_{k}\left(x_{0}\right)$ para algum $k \in\{1,2, \ldots\}$. Segue da continuidade de $f$ a existência de uma solução local $x(t)$ de $\dot{x}=f(t, x) \operatorname{com} x\left(t_{0}^{+}\right)=x_{0}$. Agora, como $\tau_{i}(x)<\tau_{j}(x)$ para $i<j$ (veja condição $C 2$ ) acima) e $t_{0}=\tau_{k}\left(x_{0}\right)$, segue que $t \neq \tau_{j}(x(t))$ para $j \neq k$ e $t$ suficientemente próximo de $t_{0}$. Por outro lado, a condição (1.10) não permite a possibilidade de termos $t=\tau_{k}(x(t))$ em uma vizinhança à direita de $t_{0}$ suficientemente pequena. Portanto $x(t)$ é uma solução local do sistema (1.8). Isto conclui a demonstração. 
A condição (1.10) é razoável somente para funções irregulares $\tau_{k}(x)$, já que o Teorema das Funções Implícitas implica que, se $\tau_{k}$ for diferenciável em $x_{0}$ e $\tau_{k}^{\prime}\left(x_{0}\right) \neq 0$, então (1.10) poderá jamais ser válida.

No resultado seguinte, estabelecemos algumas condições para as funções $\tau_{k}(x)$ para obtermos existência de soluções.

Teorema 1.1.9 Suponhamos que:

i) $f: D \rightarrow \mathbb{R}^{n}$ seja contínua;

ii) as funções $\tau_{k}: \Omega \rightarrow(0,+\infty)$ sejam diferenciáveis para cada $k=1,2,3 \ldots$;

iii) se $t_{1}=\tau_{k}\left(x_{1}\right)$ para algum $\left(t_{1}, x_{1}\right) \in D$ e para algum $k \in \mathbb{N}^{*}$, então existe $\delta>0$ tal que

$$
\frac{\partial \tau_{k}(x)}{\partial x} \cdot f(x, t) \neq 1
$$

$$
\text { para }(t, x) \in D \text { tal que }\left|x-x_{1}\right|<\delta \text { e } 0<t-t_{1}<\delta \text {. }
$$

Então para cada $\left(t_{0}, x_{0}\right) \in D$, existe uma solução $x:\left[t_{0}, t_{0}+a\right) \rightarrow \mathbb{R}^{n}$ de (1.8) para algum $a>0$.

Demonstração: Suponhamos que exista $(\bar{t}, \bar{x}) \in D$ tal que $\bar{t}=\tau_{k}(\bar{x})$ para algum $k \geq 1$. Notemos que o sistema

$$
\left\{\begin{array}{l}
\dot{x}(t)=f(x, t), \\
x(\bar{t})=\bar{x},
\end{array}\right.
$$

possui uma solução local em um intervalo $[\bar{t}, \bar{t}+a), a>0$. Definamos $\sigma:[\bar{t}, \bar{t}+a) \rightarrow \mathbb{R}$ por

$$
\sigma(t)=t-\tau_{k}(x(t))
$$

Como $\bar{t}=\tau_{k}(x(\bar{t}))$ temos $\sigma(\bar{t})=0$ e

$$
\sigma^{\prime}(t)=1-\frac{\partial \tau_{k}(x)}{\partial x} \cdot f(x, t)
$$


Pela condição iii), existe $\delta>0, \delta<a$, tal que $\sigma^{\prime}(t) \neq 0$ para todo $t \in(\bar{t}, \bar{t}+\delta)$. Assim $\sigma(t)$ é estritamente crescente ou estritamente decrescente em $(\bar{t}, \bar{t}+\delta)$. Como $\sigma(\bar{t})=0$, segue que $t \neq \tau_{k}(x(t))$ para todo $t \in(\bar{t}, \bar{t}+\delta)$. O resultado segue do teorema anterior.

Resultados sobre continuação de soluções, teoremas de unicidade e estabilidade em equações diferenciais impulsivas podem ser encontrados em [19].

\subsection{Sistemas semidinâmicos}

Nesta seção, apresentamos alguns conceitos básicos da teoria de sistemas semidinâmicos contínuos. Destacamos apenas algumas definições e resultados que serão importantes no estudo da teoria de sistemas semidinâmicos com impulsos. As referências utilizadas foram os livros [1] e [2].

Em toda esta seção, vamos considerar $(X, \rho)$ um espaço métrico.

Definição 1.2.1 Dizemos que o par $(X, \pi)$ é um sistema semidinâmico, se a aplicação

$$
\pi: X \times \mathbb{R}_{+} \rightarrow X
$$

satisfaz as seguintes propriedades:

i) $\pi(x, 0)=x$ para todo $x \in X$;

ii) $\pi(\pi(x, t), s)=\pi(x, t+s)$ para todo $t, s \in \mathbb{R}_{+}$e $x \in X$;

iii) $\pi$ é contínua em $X \times \mathbb{R}_{+}$.

Observação 1.2.2 Se Substituirmos $\mathbb{R}_{+}$por $\mathbb{R}$ na definição acima, diremos que a aplicação $\pi$ é um sistema dinâmico em $X$.

Para cada $x \in X$, a aplicação $\pi$ induz uma aplicação contínua $\pi_{x}: \mathbb{R}_{+} \rightarrow X$ definida por $\pi_{x}(t)=\pi(x, t)$. E para cada $t \in \mathbb{R}_{+}$, a aplicação $\pi$ induz uma aplicação contínua $\pi_{t}: X \rightarrow X$ definida por $\pi_{t}(x)=\pi(x, t)$. 
Apresentamos, a seguir, um exemplo clássico de sistema semidinâmico.

Exemplo 1.2.3 Consideremos a equação diferencial autônoma

$$
\frac{d x}{d t}=f(x)
$$

onde $f: \mathbb{R}^{n} \rightarrow \mathbb{R}^{n}$ é contínua. Vamos assumir que para cada $x \in \mathbb{R}^{n}$, existe uma única solução $\phi(t, x)$ de (1.12) definida em $\mathbb{R}_{+}$e satisfazendo a condição $\phi(0, x)=x$. Pela unicidade de soluções, obtemos

$$
\phi(t, \phi(s, x))=\phi(t+s, x)
$$

para todo $t, s \in \mathbb{R}_{+}$. Então a aplicação

$$
\pi: \mathbb{R}^{n} \times \mathbb{R}_{+} \rightarrow \mathbb{R}^{n}
$$

dada por $\pi(x, t)=\phi(t, x)$, define um sistema semidinâmico em $\mathbb{R}^{n}$.

Definição 1.2.4 Seja $(X, \pi)$ um sistema semidinâmico. Para qualquer $x \in X$ fixado, a órbita positiva de $x$ (ou trajetória positiva) é dada pelo conjunto

$$
\pi^{+}(x)=\left\{\pi(x, t): t \in \mathbb{R}_{+}\right\}
$$

E para qualquer subconjunto $A \subset X$ e $\Delta \subset \mathbb{R}_{+}$, definimos

$$
\pi^{+}(A)=\cup\left\{\pi^{+}(x): x \in A\right\}
$$

e

$$
\pi(A, \Delta)=\cup\{\pi(x, t): x \in A \text { e } t \in \Delta\}
$$

Definição 1.2.5 Um subconjunto $A \subset X$ será chamado positivamente $\pi$-invariante se $\pi^{+}(A) \subset A$.

Definição 1.2.6 Um ponto $x \in X$ é chamado ponto crítico se $\pi(x, t)=x$ para todo $t \in \mathbb{R}_{+}$. 
Definição 1.2.7 Um ponto $x \in X$ é chamado ponto periódico se existe $T>0$ tal que $\pi(x, T)=x$.

A seguir, apresentamos alguns resultados sobre invariância. As demonstrações podem ser encontradas em [2].

Teorema 1.2.8 Sejam $(X, \pi)$ um sistema semidinâmico e $\left\{A_{i}\right\}_{i \in I}$ uma coleção de subconjuntos positivamente $\pi$-invariantes de $X$, onde $I$ é um conjunto de índices. Então a união e a interseção destes conjuntos são positivamente $\pi$-invariantes.

Teorema 1.2.9 Sejam $(X, \pi)$ um sistema semidinâmico e $A \subset X$ um subconjunto positivamente $\pi$-invariante. Então o fecho de $A$, denotado por $\bar{A}$, é positivamente $\pi$-invariante.

Proposição 1.2.10 A órbita positiva de $x, \pi^{+}(x)$, é um conjunto positivamente $\pi$-invariante para todo $x \in X$.

\subsubsection{Conjuntos limites}

Definição 1.2.11 Sejam $(X, \pi)$ um sistema semidinâmico e $x \in X$. Um ponto $y \in X$ é chamado ponto limite positivo do ponto $x \in X$, se existe uma sequência $\left\{t_{n}\right\}_{n \geq 1} \subset \mathbb{R}_{+}$ tal que $t_{n} \stackrel{n \rightarrow+\infty}{\longrightarrow}+\infty$ de modo que $\pi\left(x, t_{n}\right) \stackrel{n \rightarrow+\infty}{\longrightarrow} y$. Chamamos o conjunto de todos os pontos limites positivos de $x$ de conjunto limite positivo de $x$ e denotamos esse conjunto por

$$
L^{+}(x)=\left\{y \in X: \text { existe }\left\{t_{n}\right\}_{n \geq 1} \subset \mathbb{R}_{+} \text {tal que } t_{n} \stackrel{n \rightarrow+\infty}{\longrightarrow}+\infty \text { e } \pi\left(x, t_{n}\right) \stackrel{n \rightarrow+\infty}{\longrightarrow} y\right\}
$$

O próximo resultado mostra uma maneira alternativa para definirmos o conjunto limite positivo.

Lema 1.2.12 Sejam $(X, \pi)$ um sistema semidinâmico e $x \in X$, então $L^{+}(x)=\bigcap_{t>0} \overline{\pi(x,[t,+\infty))}$.

Demonstração: Seja $y \in L^{+}(x)$. Então existe uma sequência $\left\{t_{n}\right\}_{n \geq 1} \subset \mathbb{R}_{+}$tal que $t_{n} \stackrel{n \rightarrow+\infty}{\longrightarrow}+\infty$ e $\pi\left(x, t_{n}\right) \stackrel{n \rightarrow+\infty}{\longrightarrow} y$. Dado $\tau \in[0,+\infty)$, existe um natural $n_{0}>0$ tal que $t_{n} \in[\tau,+\infty)$ para $n>n_{0}$. Logo

$$
\pi\left(x, t_{n}\right) \in \pi(x,[\tau,+\infty))
$$


para todo $n>n_{0}$. Portanto

$$
y \in \overline{\pi(x,[\tau,+\infty))}
$$

Como $\tau$ é arbitrário, seque que $y \in \bigcap_{t>0} \overline{\pi(x,[t,+\infty))}$. Portanto, $L^{+}(x) \subset \bigcap_{t>0} \overline{\pi(x,[t,+\infty))}$.

Agora, suponhamos $y \in \bigcap_{t>0} \overline{\pi(x,[t,+\infty))}$. Então $y \in \overline{\pi(x,[\tau,+\infty))}$ para todo $\tau>0$. Em particular, dado uma sequência $\left\{t_{n}\right\}_{n \geq 1} \subset \mathbb{R}_{+}$tal que $t_{n} \stackrel{n \rightarrow+\infty}{\longrightarrow}+\infty$, existe $\lambda_{n} \in\left[t_{n},+\infty\right)$ tal que

$$
\rho\left(\pi\left(x, \lambda_{n}\right), y\right)<\frac{1}{n}
$$

para cada $n=1,2, \ldots$ Claramente, $\lambda_{n} \stackrel{n \rightarrow+\infty}{\longrightarrow}+\infty$. Por construção, temos que $\pi\left(x, \lambda_{n}\right) \stackrel{n \rightarrow+\infty}{\longrightarrow} y$. Logo $y \in L^{+}(x)$ e portanto $\bigcap_{t>0} \overline{\pi(x,[t,+\infty))} \subset L^{+}(x)$. Isto prova o lema.

Exemplo 1.2.13 Consideremos o sistema em coordenadas polares em $\mathbb{R}^{2}$

$$
\left\{\begin{array}{l}
\dot{r}=r(1-r), \\
\dot{\theta}=1
\end{array}\right.
$$

O retrato de fase do sistema (1.13) consiste da trajetória fechada $\gamma$ coincidindo com o círculo unitário $r=1$, do ponto dado por $r=0$, e de trajetórias espirais que se aproximam do círculo $\gamma$, quando $t \rightarrow+\infty$. Veja a Figura 1.10 .

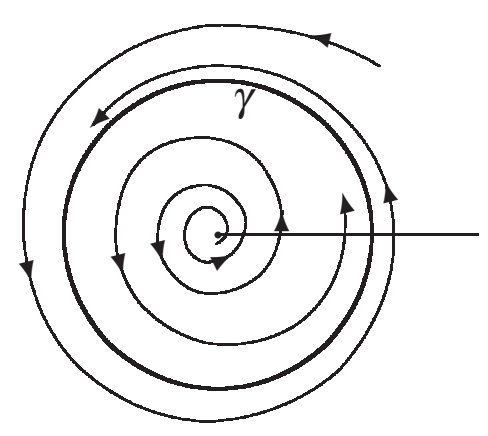

Figura 1.10: Curva integral do sistema (1.13). 
Exceto para a trajetória $r=0$, a trajetória fechada $\gamma$ é o conjunto limite positivo de todos os pontos do espaço de fase com $r>0$ para o sistema (1.13). O conjunto limite positivo do ponto $r=0$ é o ponto $r=0$.

Teorema 1.2.14 Sejam $(X, \pi)$ um sistema semidinâmico e $x \in X$. Então valem as seguintes propriedades:

i) $\overline{\pi^{+}(x)}=\pi^{+}(x) \cup L^{+}(x)$.

ii) $\mathrm{L}^{+}(x)$ é fechado e positivamente $\pi$-invariante.

iii) Se $X$ for localmente compacto e $L^{+}(x)$ for compacto e não vazio, então $L^{+}(x)$ será conexo.

Demonstração: i) É suficiente mostrarmos que $\overline{\pi^{+}(x)} \subset \pi^{+}(x) \cup L^{+}(x)$. Seja $y \in \overline{\pi^{+}(x)}$ então existe uma sequência $\left\{y_{n}\right\}_{n \geq 1} \subset \pi^{+}(x)$ tal que $y_{n} \stackrel{n \rightarrow+\infty}{\longrightarrow} y$. Como $\left\{y_{n}\right\}_{n \geq 1} \subset \pi^{+}(x)$, para cada $n \in \mathbb{N}$, existe $t_{n} \in \mathbb{R}_{+}$tal que $y_{n}=\pi\left(x, t_{n}\right)$. Se $t_{n} \stackrel{n \rightarrow+\infty}{\longrightarrow}+\infty$ segue que $y \in L^{+}(x)$. Caso contrário, existe $\tau \in \mathbb{R}_{+}$tal que $t_{n} \stackrel{n \rightarrow+\infty}{\longrightarrow} \tau$. Pela continuidade da aplicação $\pi$ temos

$$
\pi\left(x, t_{n}\right) \stackrel{n \rightarrow+\infty}{\longrightarrow} \pi(x, \tau)=y,
$$

e portanto, $y \in \pi^{+}(x)$. Isto mostra que $\overline{\pi^{+}(x)} \subset \pi^{+}(x) \cup L^{+}(x)$ e o resultado está provado.

ii) Pelo Lema 1.2.12, $L^{+}(x)$ é fechado. Mostremos que $L^{+}(x)$ é positivamente $\pi$-invariante. Seja $y \in L^{+}(x)$. Logo existe uma sequência $\left\{t_{n}\right\}_{n \geq 1} \subset \mathbb{R}_{+}$tal que $t_{n} \stackrel{n \rightarrow+\infty}{\longrightarrow}+\infty$ e $\pi\left(x, t_{n}\right) \stackrel{n \rightarrow+\infty}{\longrightarrow} y$. Agora, seja $\tau \geq 0$ arbitrário. Então pela continuidade da $\pi$, segue que

$$
\pi\left(x, t_{n}+\tau\right)=\pi\left(\pi\left(x, t_{n}\right), \tau\right) \stackrel{n \rightarrow+\infty}{\longrightarrow} \pi(y, \tau) \in L^{+}(x)
$$

Portanto $\pi^{+}(y) \subset L^{+}(x)$ para todo $y \in L^{+}(x)$.

iii) Suponhamos que $L^{+}(x)$ não seja conexo. Então $L^{+}(x)=A \cup B$, onde $A$ e $B$ são não vazios, fechados e disjuntos. Como $L^{+}(x)$ é compacto, segue que $A$ e $B$ são compactos. Daí, pelo fato de $X$ ser localmente compacto, existe um $\varepsilon>0$ de modo que $\overline{B(A, \varepsilon)}$ e $\overline{B(B, \varepsilon)}$ sejam compactos e disjuntos. Sejam $a \in A$ e $b \in B$. Então existem sequências reais $\left\{t_{n}\right\}_{n \geq 1}$ e $\left\{h_{n}\right\}_{n \geq 1}$, com $t_{n} \stackrel{n \rightarrow+\infty}{\longrightarrow}+\infty$ e $h_{n} \stackrel{n \rightarrow+\infty}{\longrightarrow}+\infty$, tais que $\pi\left(x, t_{n}\right) \stackrel{n \rightarrow+\infty}{\longrightarrow} a$ e $\pi\left(x, h_{n}\right) \stackrel{n \rightarrow+\infty}{\longrightarrow} b$. Podemos supor, sem perda de generalidade, 
que $\pi\left(x, t_{n}\right) \in B(A, \varepsilon), \pi\left(x, h_{n}\right) \in B(B, \varepsilon)$ e $h_{n}-t_{n}>0$ para todo $n \in \mathbb{N}$. Como os segmentos de trajetórias $\pi(x, t), t_{n} \leq t \leq h_{n}, n=1,2,3, \ldots$, são conjuntos conexos e compactos, eles claramente interceptam $\partial B(A, \varepsilon)$ e $\partial B(B, \varepsilon)$. Em particular, existe uma sequência $\left\{T_{n}\right\}_{n \geq 1}, t_{n}<T_{n}<h_{n}$, de maneira que $\pi\left(x, T_{n}\right) \in \partial B(A, \varepsilon)$, o qual é compacto. Deste modo, podemos assumir que $\pi\left(x, T_{n}\right) \stackrel{n \rightarrow+\infty}{\longrightarrow} z \operatorname{com} z \in \partial B(A, \varepsilon)$. Mas $T_{n} \stackrel{n \rightarrow+\infty}{\longrightarrow}+\infty$ e assim $z \in L^{+}(x)$, o que é um absurdo. Portanto $L^{+}(x)$ é necessariamente conexo.

Na próxima definição, apresentamos os conceitos de prolongamento do conjunto limite positivo e conjunto prolongado.

Definição 1.2.15 Sejam $(X, \pi)$ um sistema semidinâmico e $x \in X$. O prolongamento do conjunto limite positivo de $x$ é dado por

$$
\begin{gathered}
J^{+}(x)=\left\{y \in X: \text { existem }\left\{x_{n}\right\}_{n \geq 1} \subset X \text { e }\left\{t_{n}\right\}_{n \geq 1} \subset \mathbb{R}_{+} \text {tais que } x_{n} \stackrel{n \rightarrow+\infty}{\longrightarrow} x\right. \\
\left.t_{n} \stackrel{n \rightarrow+\infty}{\longrightarrow}+\infty \text { e } \pi\left(x_{n}, t_{n}\right) \stackrel{n \rightarrow+\infty}{\longrightarrow} y\right\} .
\end{gathered}
$$

O conjunto prolongado de $x$ é dado por

$D^{+}(x)=\left\{y \in X:\right.$ existem $\left\{x_{n}\right\}_{n \geq 1} \subset X$ e $\left\{t_{n}\right\}_{n \geq 1} \subset \mathbb{R}_{+}$tais que $x_{n} \stackrel{n \rightarrow+\infty}{\longrightarrow} x$ e $\left.\pi\left(x_{n}, t_{n}\right) \stackrel{n \rightarrow+\infty}{\longrightarrow} y\right\}$.

O próximo resultado mostra uma maneira alternativa para definirmos o prolongamento do conjunto limite positivo e o conjunto prolongado.

Lema 1.2.16 Seja $x \in X$. Então

$$
D^{+}(x)=\bigcap_{\varepsilon>0} \overline{\bigcup_{t \geq 0} \pi(B(x, \varepsilon), t)} \text { e } \quad J ^ { + } ( x ) = \bigcap _ { \varepsilon > 0 } \longdiv { \bigcup \{ \pi ( B ( x , \varepsilon ) , \tau ) : \tau \geq t \} } .
$$

Demonstração: Mostremos a primeira igualdade. Seja $y \in \bigcap_{\varepsilon>0} \overline{\bigcup_{t \geq 0} \pi(B(x, \varepsilon), t)}$, então $y \in \overline{\bigcup_{t \geq 0} \pi(B(x, \varepsilon), t)}$ para todo $\varepsilon>0$. Logo, para cada $n \in \mathbb{N}$, existe uma sequência $\left\{y_{m}^{n}\right\}_{m \geq 1} \subset \bigcup_{t \geq 0} \pi\left(B\left(x, \frac{1}{n}\right), t\right)$ tal que $y_{m}^{n} \stackrel{m \rightarrow+\infty}{\longrightarrow} y$. Notemos que existem $t_{m}^{n} \in \mathbb{R}_{+}$e $x_{m}^{n} \in B\left(x, \frac{1}{n}\right)$ 
tais que $y_{m}^{n}=\pi\left(x_{m}^{n}, t_{m}^{n}\right)$. Pela construção, podemos obter uma subsequência $\left\{x_{m_{n}}^{n}\right\}_{n \geq 1}$ de $\left\{x_{m}^{n}\right\}_{m \geq 1}$ tal que $x_{m_{n}}^{n} \stackrel{n \rightarrow+\infty}{\longrightarrow} x$. Como $\pi\left(x_{m_{n}}^{n}, t_{m_{n}}^{n}\right) \stackrel{n \rightarrow+\infty}{\longrightarrow} y$, segue que $y \in D^{+}(x)$.

Agora, mostremos a outra inclusão. Seja $y \in D^{+}(x)$, então existem sequências $\left\{x_{n}\right\}_{n \geq 1} \subset X$ e $\left\{t_{n}\right\}_{n \geq 1} \subset \mathbb{R}_{+}$tais que $x_{n} \stackrel{n \rightarrow+\infty}{\longrightarrow} x$ e $\pi\left(x_{n}, t_{n}\right) \stackrel{n \rightarrow+\infty}{\longrightarrow} y$. Dado $\varepsilon>0$ existe $n_{0} \in \mathbb{N}$ tal que $\pi\left(x_{n}, t_{n}\right) \in \bigcup_{t>0} \pi(B(x, \varepsilon), t)$ para todo $n \geq n_{0}$. Portanto, $y \in \overline{\bigcup_{t \geq 0} \pi(B(x, \varepsilon), t)}$ para todo $\varepsilon>0$, isto é, $y \in \bigcap_{\varepsilon>0} \overline{\bigcup_{t \geq 0} \pi(B(x, \varepsilon), t)}$. Assim concluímos que

$$
D^{+}(x)=\bigcap_{\varepsilon>0} \overline{\bigcup_{t \geq 0} \pi(B(x, \varepsilon), t)}
$$

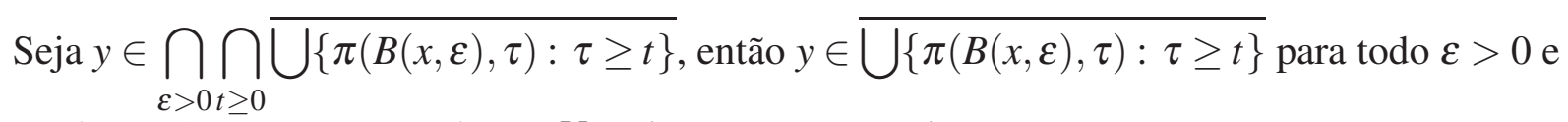
para todo $t \geq 0$. Então para cada $n \in \mathbb{N}$, existe uma sequência

$$
\left\{y_{m}^{n}\right\}_{m \geq 1} \subset \overline{\bigcup\left\{\pi\left(B\left(x, \frac{1}{n}\right), \tau\right): \tau \geq n\right\}}
$$

tal que $y_{m}^{n} \stackrel{m \rightarrow+\infty}{\longrightarrow} y$. Assim, existem $t_{m}^{n} \geq n$ e $x_{m}^{n} \in B\left(x, \frac{1}{n}\right)$ tais que $y_{m}^{n}=\pi\left(x_{m}^{n}, t_{m}^{n}\right)$. Pela construção, podemos obter uma subsequência $\left\{x_{m_{n}}^{n}\right\}_{n \geq 1}$ de $\left\{x_{m}^{n}\right\}_{m \geq 1}$ tal que $x_{m_{n}}^{n} \stackrel{n \rightarrow+\infty}{\longrightarrow} x$. Notemos que $t_{m_{n}} \stackrel{n \rightarrow+\infty}{\longrightarrow}+\infty$. Como $\pi\left(x_{m_{n}}^{n}, t_{m_{n}}^{n}\right) \stackrel{n \rightarrow+\infty}{\longrightarrow} y$, segue que $y \in J^{+}(x)$.

Por outro lado, seja $y \in J^{+}(x)$. Então existem sequências $\left\{x_{n}\right\}_{n \geq 1} \subset X$ e $\left\{t_{n}\right\}_{n \geq 1} \subset \mathbb{R}_{+}$tais que $x_{n} \stackrel{n \rightarrow+\infty}{\longrightarrow} x, t_{n} \stackrel{n \rightarrow+\infty}{\longrightarrow}+\infty$ e $\pi\left(x_{n}, t_{n}\right) \stackrel{n \rightarrow+\infty}{\longrightarrow} y$. Podemos supor, a menos de subsequência, que a sequência $\left\{t_{n}\right\}_{n \geq 1}$ é estritamente crescente. Então, dados $\varepsilon>0$ e $t \geq 0$, existe um natural $n_{0}>0$ tal que $\pi\left(x_{n}, t_{n}\right) \in \bigcup\{\pi(B(x, \varepsilon), \tau): \tau \geq t\}$ para $n \geq n_{0}$. Logo $y \in \overline{\bigcup\{\pi(B(x, \varepsilon), \tau): \tau \geq t\}}$ para todo $\varepsilon>0$ e para todo $t \geq 0$. Então $y \in \bigcap$

$$
J^{+}(x)=\bigcap_{\varepsilon>0} \bigcap \overline{\bigcup\{\pi(B(x, \varepsilon), \tau): \tau \geq t\}}
$$


Exemplo 1.2.17 Consideremos o sistema

$$
\left\{\begin{array}{l}
\dot{x}_{1}=-x_{1}, \\
\dot{x}_{2}=x_{2}
\end{array}\right.
$$

Vejamos o esboço das trajetórias do sistema (1.14) representado na Figura 1.11.

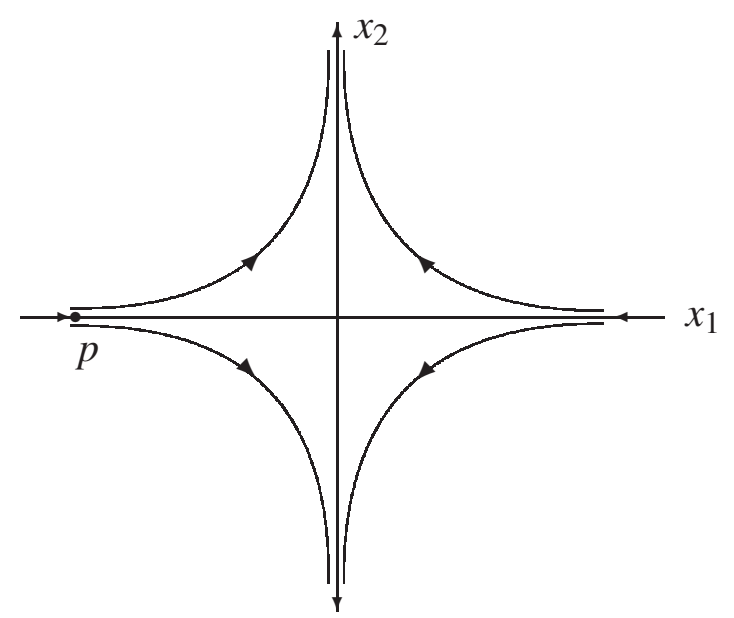

Figura 1.11: Curva integral do sistema (1.14).

Se $p$ for um ponto qualquer do eixo $x_{1}$, então $D^{+}(p)=\pi^{+}(p) \cup\left\{\left(x_{1}, x_{2}\right) \in \mathbb{R}^{2}: x_{1}=0\right\}$ e $J^{+}(p)=\left\{\left(x_{1}, x_{2}\right) \in \mathbb{R}^{2}: x_{1}=0\right\}$. Caso $p$ não esteja sobre o eixo $x_{1}$, temos $D^{+}(p)=\pi^{+}(p)$ e $J^{+}(p)=\emptyset$.

O próximo teorema mostra algumas propriedades dos conjuntos $J^{+}(x)$ e $D^{+}(x)$, para $x \in X$, onde $(X, \pi)$ é um sistema semidinâmico.

Teorema 1.2.18 Seja $(X, \pi)$ um sistema semidinâmico. Valem as propriedades:

i) Se $x \in X$, então $\overline{\pi^{+}(x)} \subset \mathrm{D}^{+}(x)$;

ii) Se $x \in X$, então $J^{+}(x)$ e $D^{+}(x)$ são fechados e positivamente $\pi$-invariantes;

iii) $D^{+}(x)=\pi^{+}(x) \cup J^{+}(x)$, para cada $x \in X$. 
Demonstração: $i)$ Seja $y \in \overline{\pi^{+}(x)}$. Logo existe uma sequência $\left\{y_{n}\right\}_{n \geq 1} \subset \pi^{+}(x)$ tal que $y_{n} \stackrel{n \rightarrow+\infty}{\longrightarrow} y$, isto é, existe uma sequência $\left\{t_{n}\right\}_{n \geq 1} \subset \mathbb{R}_{+}$tal que $y_{n}=\pi\left(x, t_{n}\right)$ e $\pi\left(x, t_{n}\right) \stackrel{n \rightarrow+\infty}{\longrightarrow} y$. Portanto, tomando a sequência $x_{n}=x$, segue que $\left\{x_{n}\right\}_{n \geq 1} \subset X, x_{n} \stackrel{n \rightarrow+\infty}{\longrightarrow} x$ e $\pi\left(x_{n}, t_{n}\right) \stackrel{n \rightarrow+\infty}{\longrightarrow} y$, donde $y \in D^{+}(x)$.

ii) Pelo Lema 1.2.16, os conjuntos $J^{+}(x)$ e $D^{+}(x)$ são fechados. A prova da invariância é análoga a demonstração do item $i i$ ) do Teorema 1.2.14.

iii) Seja $x \in X$. É fácil ver que $\pi^{+}(x) \cup J^{+}(x) \subset D^{+}(x)$. Provemos a outra inclusão. Seja $y \in D^{+}(x)$ então existem sequências $\left\{x_{n}\right\}_{n \geq 1} \subset X$ e $\left\{t_{n}\right\}_{n \geq 1} \subset \mathbb{R}_{+}$tais que $x_{n} \stackrel{n \rightarrow+\infty}{\longrightarrow} x$ e $\pi\left(x_{n}, t_{n}\right) \stackrel{n \rightarrow+\infty}{\longrightarrow} y$. Se $t_{n} \stackrel{n \rightarrow+\infty}{\longrightarrow}+\infty$, segue que $y \in J^{+}(x)$. Caso contrário, podemos assumir a menos de subsequência, que existe $t \in \mathbb{R}_{+}$tal que $t_{n} \stackrel{n \rightarrow+\infty}{\longrightarrow} t \in \mathbb{R}_{+}$. Pela continuidade da $\pi$ temos

$$
\pi\left(x_{n}, t_{n}\right) \stackrel{n \rightarrow+\infty}{\longrightarrow} \pi(x, t)
$$

Assim $\pi(x, t)=y \in \pi^{+}(x)$. Portanto $D^{+}(x)=\pi^{+}(x) \cup J^{+}(x)$ para todo $x \in X$.

\subsubsection{Estabilidade}

Nesta seção, definimos alguns conceitos de estabilidade.

Definição 1.2.19 Sejam $(X, \pi)$ um sistema semidinâmico e $A$ um subconjunto de $X$. O conjunto $A$ é chamado:

a) $\pi$-estável se, para todo $\varepsilon>0$ e todo $x \in A$, existe $\delta=\delta(x, \varepsilon)>0$ tal que $\pi^{+}(B(x, \delta)) \subset B(A, \varepsilon) ;$

b) orbitalmente $\pi$-estável se, para cada vizinhança $U$ de $A$ existe uma vizinhança positivamente $\pi$-invariante $V$ de $A$ tal que $V \subset U$;

c) $\pi$-estável segundo Bhatia e Hajek, se para todo $x \in A$ e $y \notin A$ existem vizinhanças $V$ de $x$ e $U$ de $y$ tais que $U \cap \pi(V,[0,+\infty))=\emptyset$;

d) equi $\pi$-estável se, para cada $x \notin A$ existe $\delta=\delta(x)>0$ tal que $x \notin \overline{\pi^{+}(B(A, \delta))}$; 
e) uniformemente $\pi$-estável se, para cada $\varepsilon>0$ existe $\delta=\delta(\varepsilon)>0$ tal que $\pi^{+}(B(A, \delta)) \subset B(A, \varepsilon)$.

Teorema 1.2.20 Seja $(X, \pi)$ um sistema semidinâmico. Suponhamos que $X$ seja localmente compacto e $A$ seja um subconjunto compacto de $X$. Então são equivalentes:

i) $A$ é $\pi$-estável;

ii) A é orbitalmente $\pi$-estável;

iii) A é $\pi$-estável segundo Bhatia e Hajek;

iv) A é uniformemente $\pi$-estável;

v) $A$ é equi $\pi$-estável;

vi) $D^{+}(A)=A$.

A demonstração do teorema acima pode ser encontrada em [2]. A equivalência $i) \Leftrightarrow v i$ ) é conhecida como Teorema de Ura. 


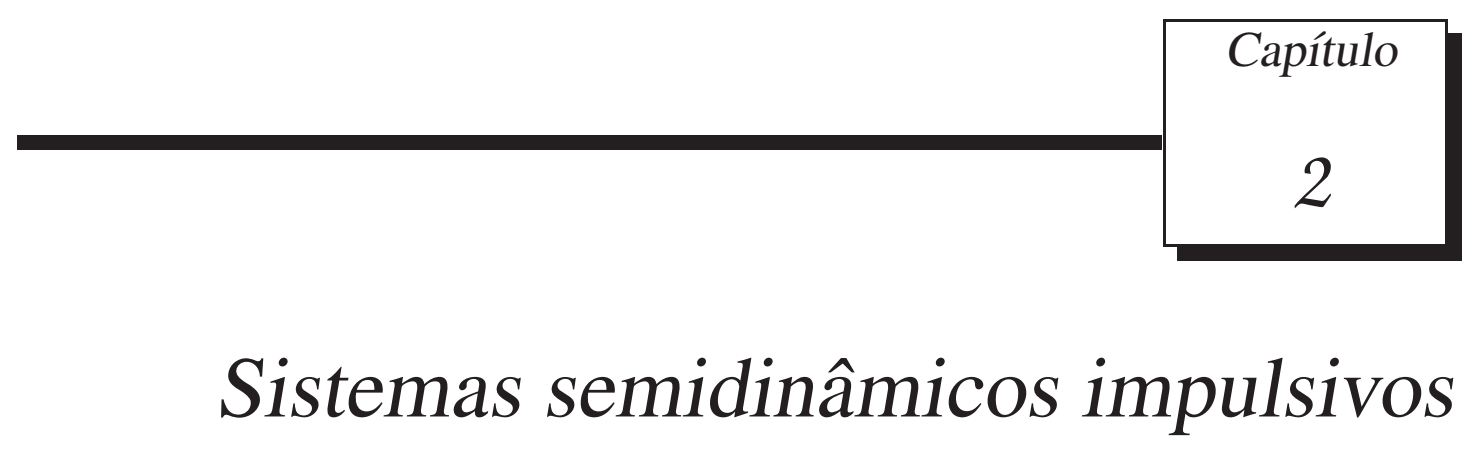

Neste capítulo, apresentamos a teoria de sistemas semidinâmicos impulsivos. Na Seção 2.1, definimos um sistema semidinâmico impulsivo. Na Seção 2.2, investigamos a continuidade da função que representa o menor tempo positivo para o qual a trajetória de um ponto encontra o conjunto impulsivo. Na Seção 2.3, apresentamos o conceito de invariância positiva para um sistema semidinâmico impulsivo e apresentamos alguns resultados. Finalizamos o capítulo, na Seção 2.4, apresentando a teoria de conjuntos limites para sistemas impulsivos.

As referências utilizadas foram [3], [5], [6], [12], [13], [15] e [18].

\subsection{Descrição de um sistema semidinâmico com impulsos}

Sejam $(X, \pi)$ um sistema semidinâmico, $t \geq 0$ e $x \in X$. Definimos o seguinte conjunto

$$
F(x, t)=\{y \in X: \pi(y, t)=x\}
$$

e, para $\Delta \subset[0,+\infty)$ e $D \subset X$, definimos

$$
F(D, \Delta)=\cup\{F(x, t): x \in D \text { e } t \in \Delta\}
$$

Definição 2.1.1 Um sistema semidinâmico impulsivo $(X, \pi ; M, I)$ consiste de um sistema semidinâmico $(X, \pi)$ juntamente com um subconjunto fechado não vazio $M$ de $X$ e uma função contínua 
$I: M \rightarrow X$ tal que a seguinte propriedade seja válida:

Para cada $x \in M$, existe um $\varepsilon_{x}>0$ tal que

$$
F\left(x,\left(0, \varepsilon_{x}\right)\right) \cap M=\emptyset \quad \text { e } \quad \pi\left(x,\left(0, \varepsilon_{x}\right)\right) \cap M=\emptyset .
$$

Chamamos o conjunto $M$ de conjunto impulsivo e a função $I$ de função impulso. Na sequência, vamos descrever como a função $I$ atua nas trajetórias do sistema impulsivo.

O lema a seguir apresenta condições para existência do menor tempo estritamente positivo para o qual a trajetória do sistema impulsivo encontra $M$.

Lema 2.1.2 Seja $(X, \pi ; M, I)$ um sistema semidinâmico impulsivo. Então, para qualquer $x \in X$ tal que $\left(\pi^{+}(x) \cap M\right) \backslash\{x\} \neq \emptyset$, existe um número real positivo $s_{1}>0$, de maneira que $\pi(x, t) \notin M$ para $0<t<s_{1}$ e $\pi\left(x, s_{1}\right) \in M$.

Demonstração: Como $\left(\pi^{+}(x) \cap M\right) \backslash\{x\} \neq \emptyset$, então existe pelo menos um $t_{1} \in \mathbb{R}_{+}$tal que $\pi\left(x, t_{1}\right) \in M$. Como $\pi_{x}: \mathbb{R}_{+} \longrightarrow X$ é contínua e $M$ é fechado e não vazio, então o subconjunto compacto $\left[0, t_{1}\right] \cap \pi_{x}^{-1}(M)$ de $\mathbb{R}_{+}$possui um menor elemento, digamos $s_{1}$, o qual satisfaz o lema.

Nas definições que seguem, $(X, \pi ; M, I)$ denota um sistema semidinâmico impulsivo e $x \in X$.

Definição 2.1.3 Definimos a função $\phi: X \rightarrow(0,+\infty]$ pela lei

$$
\phi(x)= \begin{cases}s, & \text { se } \pi(x, s) \in M \text { e } \pi(x, t) \notin M \text { para } 0<t<s \\ +\infty, & \text { se } M^{+}(x)=\emptyset\end{cases}
$$

onde $M^{+}(x)=\left(\pi^{+}(x) \cap M\right) \backslash\{x\}, x \in X$. O valor $\phi(x)$ é o menor tempo positivo para o qual a trajetória de $x$ encontra $M$.

Definição 2.1.4 Dado $x \in X$, chamamos $\pi(x, \phi(x))$ de ponto impulsivo de $x$. 
Definição 2.1.5 A trajetória impulsiva de $x$ em $(X, \pi ; M, I)$ é definida indutivamente da seguinte forma: Se $M^{+}(x)=\emptyset$, então $\widetilde{\pi}_{x}(t)=\pi(x, t)$, para todo $t \in \mathbb{R}_{+}$, e $\phi(x)=+\infty$. Porém se $M^{+}(x) \neq \emptyset$, segue do Lema 2.1.2 que existe um menor positivo $s_{0} \in \mathbb{R}_{+}$de maneira que $\pi\left(x, s_{0}\right)=x_{1} \in M$ e $\pi(x, t) \notin M$, para $0<t<s_{0}$. Então definimos, $\tilde{\pi}_{x}$ sobre $\left[0, s_{0}\right]$ como sendo

$$
\tilde{\pi}_{x}(t)= \begin{cases}\pi(x, t), & 0 \leq t<s_{0} \\ x_{1}^{+}, & t=s_{0}\end{cases}
$$

onde $x_{1}^{+}=I\left(x_{1}\right)$ e $\phi(x)=s_{0}$. Vamos denotar $x$ por $x_{0}^{+}$.

Como $s_{0}<+\infty$, o processo continua mas, agora, iniciando em $x_{1}^{+}$. Deste modo, caso $M^{+}\left(x_{1}^{+}\right)=\emptyset$, definimos $\tilde{\pi}_{x}(t)=\pi\left(x_{1}^{+}, t-s_{0}\right)$, para $s_{0} \leq t<+\infty$, e $\phi\left(x_{1}^{+}\right)=+\infty$. E se $M^{+}\left(x_{1}^{+}\right) \neq \emptyset$, segue, novamente do Lema 2.1.2, que existe um menor positivo $s_{1} \in \mathbb{R}_{+}$tal que $\pi\left(x_{1}^{+}, s_{1}\right)=x_{2} \in M$ e $\pi\left(x_{1}^{+}, t-s_{0}\right) \notin M$, para $s_{0}<t<s_{0}+s_{1}$. Definimos $\widetilde{\pi}_{x}$ sobre $\left[s_{0}, s_{0}+s_{1}\right]$ por

$$
\tilde{\pi}_{x}(t)= \begin{cases}\pi\left(x_{1}^{+}, t-s_{0}\right), & s_{0} \leq t<s_{0}+s_{1}, \\ x_{2}^{+}, & t=s_{0}+s_{1},\end{cases}
$$

onde $x_{2}^{+}=I\left(x_{2}\right)$ e $\phi\left(x_{1}^{+}\right)=s_{1}$.

Suponhamos, agora, que $\widetilde{\pi}_{x}$ esteja definida no intervalo $\left[t_{n-1}, t_{n}\right]$ e que $\widetilde{\pi}_{x}\left(t_{n}\right)=x_{n}^{+}$, onde $t_{n}=\sum_{i=0}^{n-1} s_{i}, n \geq 1$. Se $M^{+}\left(x_{n}^{+}\right)=\emptyset$, então $\widetilde{\pi}_{x}(t)=\pi\left(x_{n}^{+}, t-t_{n}\right)$, para $t_{n} \leq t<+\infty$ e $\phi\left(x_{n}^{+}\right)=+\infty$. Se $M^{+}\left(x_{n}^{+}\right) \neq \emptyset$, então existe $s_{n} \in \mathbb{R}_{+}$tal que $\pi\left(x_{n}^{+}, s_{n}\right)=x_{n+1} \in M$ e $\pi\left(x_{n}^{+}, t-t_{n}\right) \notin M$, para $t_{n}<t<t_{n+1}$. Além disso,

$$
\tilde{\pi}_{x}(t)= \begin{cases}\pi\left(x_{n}^{+}, t-t_{n}\right), & t_{n} \leq t<t_{n+1}, \\ x_{n+1}^{+}, & t=t_{n+1},\end{cases}
$$

onde $x_{n+1}^{+}=I\left(x_{n+1}\right)$ e $\phi\left(x_{n}^{+}\right)=s_{n}$.

Observemos que $\tilde{\pi}_{x}$ está definida sobre cada $\left[t_{n}, t_{n+1}\right]$, onde $t_{n+1}=\sum_{i=0}^{n} s_{i}$, ou seja, $\tilde{\pi}_{x}$ está definida sobre $\left[0, t_{n+1}\right]$. 
O processo acima termina após um número finito de passos, se $M^{+}\left(x_{n}^{+}\right)=\emptyset$ para algum $n \in \mathbb{N}$, ou ele continua indefinidamente, se $M^{+}\left(x_{n}^{+}\right) \neq \emptyset, n=1,2,3, \ldots$, e neste caso $\tilde{\pi}_{x}$ está definida no intervalo $[0, T(x))$, onde $T(x)=\sum_{i=0}^{\infty} s_{i}$ e $x \in X$. Representamos a órbita positiva impulsiva ou trajetória impulsiva de $x \in X$ pelo conjunto

$$
\tilde{\pi}^{+}(x)=\{\tilde{\pi}(x, t): t \in[0, T(x))\} .
$$

Notemos que, dado qualquer $x \in X$, uma das três condições será válida:

a) $M^{+}(x)=\emptyset$ e, desta forma, a trajetória de $x$ não possui descontinuidades.

b) Para algum $n \geq 1, x_{k}^{+}$está definida, para $k=1,2, \ldots, n$, e $M^{+}\left(x_{n}^{+}\right)=\emptyset$. Neste caso, a trajetória de $x$ possue um número finito de descontinuidades.

c) Para qualquer $n \geq 1, x_{n}^{+}$está definida e $M^{+}\left(x_{n}^{+}\right) \neq \emptyset$. Neste caso, a trajetória de $x$ admite um número infinito de descontinuidades.

Definição 2.1.6 Dados $A \subset X$ e $\Delta \subset \mathbb{R}_{+}$definimos

$$
\tilde{\pi}^{+}(A)=\cup\left\{\tilde{\pi}^{+}(x): x \in A\right\} \quad \text { e } \quad \tilde{\pi}(A, \Delta)=\cup\{\tilde{\pi}(x, t): x \in A \text { e } t \in \Delta\} .
$$

Observação 2.1.7 Escrevemos $\tilde{\pi}_{x}(t)=\tilde{\pi}(x, t)$, para qualquer $t$ em $[0, T(x))$. Pela descrição do sistema impulsivo temos que para qualquer $t$ tal que $0 \leq t<T(x)$, existe $k \in\{1,2,3, \ldots\}$ tal que $t=\sum_{i=0}^{k-1} \phi\left(x_{i-1}^{+}\right)+t^{\prime}$, onde $x=x_{0}^{+}$e $\phi\left(x_{-1}^{+}\right)=0$. Consequentemente, $\tilde{\pi}(x, t)=\pi\left(x_{k-1}^{+}, t^{\prime}\right)$ para $0 \leq t^{\prime}<\phi\left(x_{k-1}^{+}\right)$.

A proposição seguinte nos diz que $\tilde{\pi}$ satisfaz o princípio da identidade e a condição de semigrupo.

Proposição 2.1.8 Seja $(X, \pi ; M, I)$ um sistema semidinâmico impulsivo. Se $x \in X$, então:

i) $\tilde{\pi}(x, 0)=x$;

ii) $\tilde{\pi}(\tilde{\pi}(x, t), s)=\tilde{\pi}(x, t+s)$ para todos $t, s \in[0, T(x))$ tais que $t+s \in[0, T(x))$. 
Demonstração: Se $\tilde{\pi}$ for contínua, a conclusão é imediata.

i) Dado $x \in X$, temos que $\tilde{\pi}(x, t)=\pi(x, t)$ para $0 \leq t<\phi(x)$. Então $\tilde{\pi}(x, 0)=\pi(x, 0)=x$.

ii) Sejam $t, s \in[0, T(x))$ tais que $t+s \in[0, T(x))$, e consideremos $y=\tilde{\pi}(x, t)$. Pela Observação 2.1.7, podemos escrever

$$
\tilde{\pi}(x, t)=\pi\left(x_{k-1}^{+}, t^{\prime}\right)
$$

para algum $k \geq 1$, onde $t=\sum_{j=0}^{k-1} \phi\left(x_{j-1}^{+}\right)+t^{\prime}$ e $0 \leq t^{\prime}<\phi\left(x_{k-1}^{+}\right)$(lembremos que estamos denotando $x=x_{0}^{+}$e $\left.\phi\left(x_{-1}^{+}\right)=0\right)$. De mesma forma, podemos escrever

$$
\widetilde{\pi}(y, s)=\pi\left(y_{\ell-1}^{+}, s^{\prime}\right)
$$

para algum $\ell \geq 1$, onde $s=\sum_{j=0}^{\ell-1} \phi\left(y_{j-1}^{+}\right)+s^{\prime}$ e $0 \leq s^{\prime}<\phi\left(y_{\ell-1}^{+}\right)\left(\operatorname{com} y=y_{0}^{+}\right.$e $\left.\phi\left(y_{-1}^{+}\right)=0\right)$.

Como $y=\tilde{\pi}(x, t)=\pi\left(x_{k-1}^{+}, t^{\prime}\right)$, obtemos

$$
\phi(y)=\phi\left(x_{k-1}^{+}\right)-t^{\prime}, \quad y_{j}^{+}=x_{k-1+j}^{+} \quad \text { e } \quad \phi\left(y_{j}^{+}\right)=\phi\left(x_{k-1+j}^{+}\right)
$$

para todo $j \geq 1$. Segue que

$$
\begin{aligned}
s & =\phi\left(y_{0}^{+}\right)+\phi\left(y_{1}^{+}\right)+\ldots+\phi\left(y_{\ell-2}^{+}\right)+s^{\prime} \\
& =\phi\left(x_{k-1}^{+}\right)-t^{\prime}+\phi\left(x_{k}^{+}\right)+\ldots+\phi\left(x_{k+\ell-3}^{+}\right)+s^{\prime} .
\end{aligned}
$$

Então,

$$
t+s=\left(\sum_{j=0}^{k+\ell-2} \phi\left(x_{j-1}^{+}\right)\right)+s^{\prime} \quad \text { e } \quad 0 \leq s^{\prime}<\phi\left(x_{k+\ell-2}^{+}\right) .
$$

Portanto, se $\ell=1$, então

$$
\tilde{\pi}(\tilde{\pi}(x, t), s)=\tilde{\pi}(y, s)=\pi\left(x_{k-1}^{+}, t^{\prime}+s\right)=\tilde{\pi}(x, t+s)
$$

e se $\ell>1$ temos $\tilde{\pi}(\tilde{\pi}(x, t), s)=\tilde{\pi}(y, s)=\pi\left(y_{\ell-1}^{+}, s^{\prime}\right)=\pi\left(x_{k+\ell-2}^{+}, s^{\prime}\right)=\tilde{\pi}(x, t+s)$.

Observação 2.1.9 É importante notarmos que se $x \in M$ então $\tilde{\pi}(x, t)=\pi(x, t)$ para todo $0 \leq t<\phi(x)$, ou seja, não há impulso no instante $t=0$. Para que a trajetória de um ponto $x \in X$ 
sofra impulso, devemos encontrar o menor tempo estritamente positivo para o qual esta trajetória encontra $M$, e neste momento ocorrerá a descontinuidade do sistema semidinâmico.

Observação 2.1.10 Vamos assumir neste trabalho que $T(x)=+\infty$ para todo $x \in X$, isto é, $\widetilde{\pi}_{x}$ estará definida para todo $t \geq 0$.

\subsection{Continuidade da função $\phi$}

Na Definição 2.1.3 da seção anterior, definimos a função $\phi$ que representa o menor tempo positivo para o qual a trajetória de um ponto $x \in X$ encontra o conjunto impulsivo $M$. No que segue, estudamos a continuidade da função $\phi$.

Definição 2.2.1 Seja $(X, \pi)$ um sistema semidinâmico. Um conjunto fechado $S$ contendo $x \in X$ é chamado de seção ou $\lambda$ - seção através de $x, \operatorname{com} \lambda$ real positivo, se existe um conjunto fechado $L$ tal que:

a) $F(L, \lambda)=S$;

b) $F(L,[0,2 \lambda])$ é uma vizinhança de $x$;

c) $F(L, \mu) \cap F(L, v)=\emptyset$, para $0 \leq \mu<v \leq 2 \lambda$.

Denominamos o conjunto $F(L,[0,2 \lambda])$ de tubo (ou $\lambda$-tubo) e o conjunto $L$ de barra.

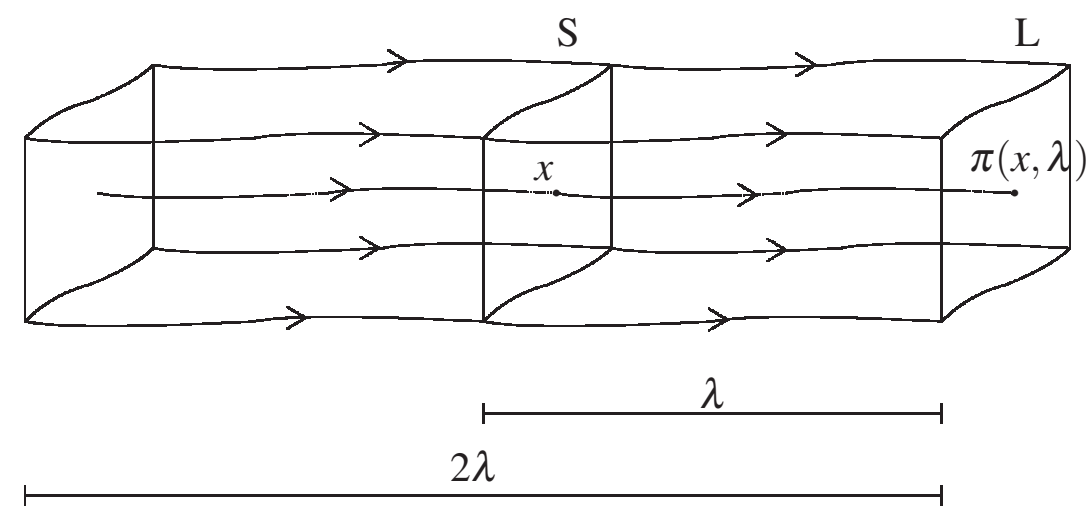

Figura 2.1: $\lambda$-tubo $F(L,[0,2 \lambda])$. 
Lema 2.2.2 Seja $(X, \pi)$ um sistema semidinâmico. Se $S$ é uma $\lambda$-seção através de $x, x \in X$, e $\mu \leq \lambda$, então $S$ também é uma $\mu$-seção através de $x$.

Demonstração: Consideremos o conjunto $L_{\mu}=F\left(L_{\lambda}, \lambda-\mu\right)$, onde $L_{\lambda}$ é uma barra do $\lambda$-tubo. Note que $L_{\mu}$ é fechado, pois $\pi$ é contínua. Mostremos que as três condições da Definição 2.2.1 são satisfeitas.

a) Temos que $F\left(\mathrm{~L}_{\mu}, \mu\right)=S$, pois

$$
\begin{gathered}
x \in F\left(L_{\mu}, \mu\right) \Leftrightarrow \pi(x, \mu) \in L_{\mu}=F\left(L_{\lambda}, \lambda-\mu\right) \Leftrightarrow \pi(\pi(x, \mu), \lambda-\mu) \in L_{\lambda} \Leftrightarrow \\
\Leftrightarrow \pi(x, \lambda) \in L_{\lambda} \Leftrightarrow x \in F\left(L_{\lambda}, \lambda\right)=S .
\end{gathered}
$$

b) Por hipótese o conjunto $F\left(L_{\lambda},[0,2 \lambda]\right)$ é uma vizinhança de $x$. Então existe um subconjunto aberto $U_{1}$ de $x$ tal que $x \in U_{1} \subset F\left(L_{\lambda},[0,2 \lambda]\right)$. Consideremos o conjunto

$$
T=F\left(L_{\lambda},[0, \lambda-\mu] \cup[\lambda+\mu, 2 \lambda]\right)
$$

Notemos que $T$ é fechado, pois dada uma sequência $\left\{y_{n}\right\}_{n \geq 1} \subset T$, com $y_{n} \stackrel{n \rightarrow+\infty}{\longrightarrow} y$, existe uma sequência $\left\{t_{n}\right\}_{n \geq 1} \subset[0, \lambda-\mu] \cup[\lambda+\mu, 2 \lambda]$ tal que $\pi\left(y_{n}, t_{n}\right) \in L_{\lambda}$. Como $[0, \lambda-\mu] \cup[\lambda+\mu, 2 \lambda]$ é compacto, podemos assumir, sem perda de generalidade, que $t_{n} \stackrel{n \rightarrow+\infty}{\longrightarrow} \tau$, $\tau \in[0, \lambda-\mu] \cup[\lambda+\mu, 2 \lambda]$. Segue da continuidade da $\pi$ que

$$
\pi\left(y_{n}, t_{n}\right) \stackrel{n \rightarrow+\infty}{\longrightarrow} \pi(y, \tau)
$$

Como $L_{\lambda}$ é fechado, temos $\pi(y, \tau) \in L_{\lambda}$. Portanto $y \in T$. Por outro lado, como $S \subset T^{c}$, onde $T^{c}$ denota o complementar de $T$ em $X$, existe um aberto $U_{2}$ contendo $x$ tal que $T \cap U_{2}=\emptyset$. Agora, mostremos que $x \in U_{1} \cap U_{2} \subset F\left(L_{\mu},[0,2 \mu]\right)$. Seja $y \in U_{1} \cap U_{2}$. Temos $U_{2} \subset T^{c}$ e $U_{1} \subset F\left(L_{\lambda},[0,2 \lambda]\right)$ então, $\pi(y, t) \in L_{\lambda}$ para algum $0 \leq t \leq 2 \lambda$ Mas, como $y \in T^{c}$ segue que

$$
\lambda-\mu<t<\lambda+\mu
$$


Tomemos $s=t+\mu-\lambda$. Como $\lambda-\mu<t<\lambda+\mu$ segue que

$$
t+\mu-\lambda>0 \text { e } \quad t+\mu-\lambda<2 \mu \text {. }
$$

Como $\pi(\pi(y, t+\mu-\lambda), \lambda-\mu)=\pi(y, t) \in L_{\lambda}$ então, $\pi(y, t+\mu-\lambda) \in L_{\mu}$ e portanto $y \in F\left(L_{\mu},[0,2 \mu]\right)$. Isto conclui a prova.

c) Suponhamos que $F\left(L_{\mu}, \sigma\right) \cap F\left(L_{\mu}, v\right) \neq \emptyset$, para $0 \leq \sigma<v \leq 2 \mu$. Desta forma, existe $y \in F\left(L_{\mu}, \sigma\right) \cap F\left(L_{\mu}, v\right)$, isto é,

$$
\pi(y, \sigma) \in L_{\mu}=F\left(L_{\lambda}, \lambda-\mu\right) \quad \text { e } \quad \pi(y, v) \in L_{\mu}=F\left(L_{\lambda}, \lambda-\mu\right) .
$$

Logo,

$$
\pi(y, \sigma+\lambda-\mu) \in L_{\lambda} \quad \text { e } \quad \pi(y, v+\lambda-\mu) \in L_{\lambda}
$$

o que é um absurdo, pois $0 \leq \sigma+\lambda-\mu<v+\lambda-\mu \leq 2 \lambda$ e $S$ é uma $\lambda$-seção por hipótese.

A demonstração está, portanto, terminada.

Na próxima definição, apresentamos as condições TC e STC para um tubo.

Definição 2.2.3 Seja $(X, \pi)$ um sistema semidinâmico. Qualquer tubo $F(L,[0,2 \lambda])$ dado pela seção $S$ através de $x \in X$ tal que

$$
S \subset M \cap F(L,[0,2 \lambda])
$$

é chamado um TC-tubo através de $x$. Dizemos que um ponto $x \in M$ satisfaz a Condição de Tubo e escrevemos abreviadamente (TC), se existir um TC-tubo, $F(L,[0,2 \lambda])$, através de $x$.

Se $S=M \cap F(L,[0,2 \lambda])$, dizemos que $F(L,[0,2 \lambda])$ é um STC-tubo através de $x$. Dizemos que um ponto $x \in M$ satisfaz a Condição Forte de Tubo e escrevemos abreviadamente (STC), se existir um STC-tubo, $F(L,[0,2 \lambda])$, através de $x$.

Exemplo 2.2.4 Consideremos o sistema semidinâmico em $\mathbb{R}^{2}$ dado por $\pi((x, y), t)=(x+t, y)$ e $M=\left\{(x, y) \in \mathbb{R}^{2}: x=0\right\} \cup\left\{(x, y) \in \mathbb{R}^{2}: x=y, x \geq 0\right\}$, veja a Figura 2.2. O ponto $(0,0)$ satisfaz a condição TC mas não satisfaz a condição STC. 


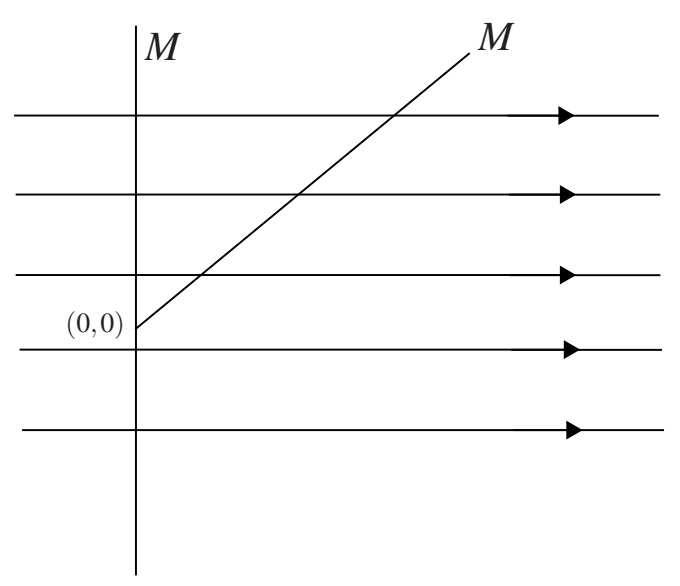

Figura 2.2.

O próximo resultado é consequência do Lema 2.2.2 e das definições acima. O leitor pode encontrar a demonstração em [13].

Lema 2.2.5 Seja $(X, \pi ; M, I)$ um sistema semidinâmico impulsivo. Suponhamos que existe um ponto $x \in X$ que satisfaça a condição TC (STC) com uma $\lambda$-seção $S$. Então, para qualquer $\eta<\lambda$, o conjunto $S$ também será uma $\eta$-seção com um TC-tubo (STC-tubo).

Definição 2.2.6 Uma função $f: X \rightarrow \mathbb{R}$ é denominada semicontínua superiormente em um ponto $a \in X$ quando, para cada $\varepsilon>0$ dado, existe $\delta>0$ tal que $x \in X$ e $\rho(x, a)<\delta$ implicam que $f(x)<f(a)+\varepsilon$. Dizemos que $f$ é semicontínua superiormente quando ela o for em cada ponto de $X$. Analogamente, $f$ é chamada semicontínua inferiormente em um ponto $a \in X$ quando, para cada $\varepsilon>0$ dado, existe $\delta>0$ tal que $x \in X$ e $\rho(x, a)<\delta$ implicam que $f(x)>f(a)-\varepsilon$. Em termos de sequências, $f$ é uma função semicontínua superiormente em $a \in X$ se, para toda sequência $\left\{x_{n}\right\}_{n \geq 1} \subset X$, com $x_{n} \stackrel{n \rightarrow+\infty}{\longrightarrow} a$, tivermos limsup $f\left(x_{n}\right) \leq f(a)$. De mesma forma, $f$ é uma função semicontínua inferiormente em $a \in X$ se, para toda sequência $\left\{x_{n}\right\}_{n \geq 1} \subset X$, com $x_{n} \stackrel{n \rightarrow+\infty}{\longrightarrow} a$, tivermos $\liminf _{n \rightarrow+\infty} f\left(x_{n}\right) \geq f(a)$. Dizemos, então, que $f$ é contínua num ponto se, e somente se, ela for contínua superiormente e inferiormente neste ponto.

Exemplo 2.2.7 Consideremos o sistema semidinâmico em $\mathbb{R}$ dado por $\pi(x, t)=x+t$. Assim, consideremos o sistema impulsivo associado com $M=\mathbb{N}$ e $I(n)=n+\frac{1}{2}$ para cada $n \in \mathbb{N}$. Então

$$
\phi(x)=\left\{\begin{array}{lll}
-x & \text { se } & x<0 \\
1+E(x)-x & \text { se } & x \geq 0
\end{array}\right.
$$


onde $E(x)$ denota a parte inteira de $x$. Notemos que a função $\phi$ não é semicontínua inferiormente nos pontos pertencentes ao conjunto $\mathbb{N}$. Com efeito, dado $x \in \mathbb{N}$, consideremos a sequência dada por $x_{n}=x-\frac{1}{n}$ para todo $n \in \mathbb{N}^{*}$. Então $x_{n} \stackrel{n \rightarrow+\infty}{\longrightarrow} x \mathrm{e}$

$$
\liminf _{n \rightarrow+\infty} \phi\left(x_{n}\right)=\liminf _{n \rightarrow+\infty}[1+(x-1)-(x-1 / n)]=0<\phi(x)=1 .
$$

Notemos que todo elemento $x \in \mathbb{N}$ satisfaz a condição STC.

O teorema seguinte diz que, se $x \notin M$, então $\phi$ é semicontínua inferiormente em $x$.

Teorema 2.2.8 Seja $(X, \pi ; M, I)$ um sistema semidinâmico impulsivo. Então, a função $\phi$ é semicontínua inferiormente em qualquer $x \in X \backslash M$,

Demonstração: Sejam $x \in X \backslash M$ e $\phi(x)=c \in(0,+\infty)$. Seja $\left\{x_{n}\right\}_{n \geq 1} \subset X$ uma sequência qualquer tal que $x_{n} \stackrel{n \rightarrow+\infty}{\longrightarrow} x$ e $\phi\left(x_{n}\right) \stackrel{n \rightarrow+\infty}{\longrightarrow} t$. Como $x \notin M$ então, para $n \in \mathbb{N}$ suficientimente grande, temos $x_{n} \notin M$. Entretanto $\pi\left(x_{n}, \phi\left(x_{n}\right)\right) \in M$ e, como $M$ é fechado e $\pi$ é contínua, segue que

$$
\pi\left(x_{n}, \phi\left(x_{n}\right)\right) \stackrel{n \rightarrow+\infty}{\longrightarrow} \pi(x, t) \in M .
$$

Logo $c=\phi(x) \leq t$. Portanto $\phi$ é semicontínua inferiormente em $x$.

Se $x \in M$ e $x$ não é um ponto inicial, então $\phi$ não é semicontínua inferiormente em $x$. O próximo resultado mostra este fato.

Teorema 2.2.9 Seja $(X, \pi ; M, I)$ um sistema semidinâmico impulsivo e suponhamos que $x \in M$ não seja um ponto inicial. Então a função $\phi$ não é semicontínua inferiormente em $x$.

Demonstração: Seja $x \in M$ e suponhamos que $x$ não seja um ponto inicial. Então existem $\varepsilon>0$ e $y \in X$ tais que $\pi(y, \varepsilon)=x$. Podemos supor $\pi(y,[0, \varepsilon)) \cap M=\emptyset$. Agora, consideremos uma sequência crescente de números reais $\left\{\varepsilon_{n}\right\}_{n \geq 1}$ tal que $\varepsilon_{n}>0$ para todo $n \in \mathbb{N}$ e $\varepsilon_{n} \stackrel{n \rightarrow+\infty}{\longrightarrow} \varepsilon$. Definindo $y_{n}=\pi\left(y, \varepsilon_{n}\right)$ para cada $n \in \mathbb{N}$, temos que $y_{n} \stackrel{n \rightarrow+\infty}{\longrightarrow} x \mathrm{e}$

$$
\phi\left(y_{n}\right)=\phi\left(\pi\left(y, \varepsilon_{n}\right)\right)=\varepsilon-\varepsilon_{n} \stackrel{n \rightarrow+\infty}{\longrightarrow} 0<\phi(x) .
$$


Portanto $\phi$ não é semicontínua inferiormente em $x$.

No Teorema 2.2.10 a seguir, vamos mostrar a condição que um ponto pertencente ao conjunto $M$ deve satisfazer para que $\phi$ seja semicontínua superiormente nele.

Teorema 2.2.10 Se, em um sistema semidinâmico impulsivo $(X, \pi ; M, I)$, cada ponto pertencente ao conjunto impulsivo $M$ satisfaz a condição (TC) então $\phi$ é semicontínua superiormente em $X$.

Demonstração: Seja $x \in X$. Podemos assumir que $\phi(x)=u \in(0,+\infty)$. Neste caso, $\pi(x, u)=y \in M$ e $\pi(x,(0, u)) \cap M=\emptyset$. Pelo Lema 2.2.5, podemos tomar $\varepsilon<u$ tal que $U=F(L,[0,2 \varepsilon])$ seja um TC-tubo com uma $\varepsilon$-seção através de $y$ igual a $F(L, \varepsilon)$. Então existe uma vizinhança $V$ de $x$ tal que $\pi(V, u) \subset U$. Assim, $\pi(z, u) \in F(L,[0,2 \varepsilon])$, para qualquer $z \in V$. Além disso, dado qualquer $z \in V$, existe um $\eta_{z} \in[0,2 \varepsilon]$ tal que $\pi\left(z, u+\eta_{z}\right) \in L$. Como $\varepsilon<u$, segue que $u+\eta_{z}-\varepsilon>0$ e

$$
\pi\left(z, u+\eta_{z}-\varepsilon\right) \in F(L, \varepsilon)=S \subset M .
$$

Daí, $\phi(z) \leq u+\eta_{z}-\varepsilon<u+\varepsilon=\phi(x)+\varepsilon$. Portanto $\phi$ é semicontínua superiormente em $x$.

O próximo resultado diz respeito à continuidade da função $\phi$.

Teorema 2.2.11 Consideremos o sistema semidinâmico impulsivo $(X, \pi ; M, I)$. Suponhamos que nenhum ponto inicial pertença ao conjunto impulsivo $M$ e que cada elemento de $M$ satisfaça a condição (TC). Então $\phi$ é contínua em $x$ se, e somente se, $x \in X \backslash M$.

\section{Demonstração:}

$(\Rightarrow)$ Suponhamos que $x \in M$. Como $x$ não é ponto inicial, segue, pelo Teorema 2.2.9, que $\phi$ não é semicontínua inferiormente. Mas isto é um absurdo. Portanto $x \in X \backslash M$.

$(\Leftarrow)$ Seja $x \in X \backslash M$. Pelo Teorema 2.2.8, $\phi$ é semicontínua inferiormente em $x$. E como cada ponto de $M$ satisfaz a condição (TC), pelo Teorema 2.2.10, $\phi$ é semicontínua superiormente em $x$. Portanto $\phi$ é contínua em $x$.

Observação 2.2.12 Vamos assumir em todo este trabalho que o conjunto impulsivo $M$ satisfaz a condição STC e que não existe ponto inicial em $M$. Assim, $\phi$ é semicontínua superiormente em $X$ e contínua em $X \backslash M$. 


\subsection{Invariância}

Semelhante ao caso contínuo, apresentamos na sequência o conceito de conjuntos positivamente invariantes.

Definição 2.3.1 Sejam $(X, \pi ; M, I)$ um sistema semidinâmico impulsivo e $A$ um subconjunto de $X$. Dizemos que $A$ é positivamente $\tilde{\pi}$-invariante se $\tilde{\pi}^{+}(A)=\cup\left\{\tilde{\pi}^{+}(x): x \in A\right\} \subset A$. Ainda, dizemos que $A$ é $I$-invariante se $I(x) \in A$ para todo $x \in M \cap A$.

O exemplo seguinte mostra que em geral não existe relação entre $\pi$-invariância, $\widetilde{\pi}$-invariância e I-invariância.

Exemplo 2.3.2 Consideremos o sistema semidinâmico impulsivo em $\mathbb{R}$ dado pelo sistema semidinâmico $\pi(x, t)=t+x, M=\{1\}$ e $I(1)=-1$. Então o conjunto $A=[0,+\infty)$ é positivamente $\pi$-invariante mas não é positivamente $\tilde{\pi}$-invariante e nem $I$-invariante. Entretanto, o conjunto $B=[-1,1)$ é positivamente $\widetilde{\pi}$-invariante mas não é positivamente $\pi$-invariante. O conjunto $C=[-2,2]$ é $I$-invariante mas não é positivamente $\widetilde{\pi}$-invariante. Já o conjunto $D=[1,+\infty)$ é positivamente $\widetilde{\pi}$-invariante mas não é $I$-invariante.

A seguir, apresentamos quatro resultados sobre invariância.

Proposição 2.3.3 Seja $(X, \pi ; M, I)$ um sistema semidinâmico impulsivo. A órbita positiva impulsiva $\tilde{\pi}^{+}(x)$ é positivamente $\tilde{\pi}$-invariante para todo $x \in X$.

Proposição 2.3.4 Sejam $(X, \pi ; M, I)$ um sistema semidinâmico impulsivo e $A$ um subconjunto de $X$ positivamente $\pi$-invariante e $I$-invariante. Então $A$ é positivamente $\tilde{\pi}$-invariante.

Demonstração: Seja $x \in A$. Então $\tilde{\pi}(x,[0, \phi(x)))=\pi(x,[0, \phi(x))) \subset A$ e $x_{1}=\pi(x, \phi(x)) \in A \cap$ M. Como $A$ é $I$-invariante, segue que $x_{1}^{+}=I\left(x_{1}\right) \in A$. Logo, $\tilde{\pi}(x,[0, \phi(x)]) \subset A$. Do mesmo modo, $\tilde{\pi}\left(x,\left[\phi(x), \phi(x)+\phi\left(x_{1}^{+}\right)\right)\right)=\pi\left(x_{1}^{+},\left[0, \phi\left(x_{1}^{+}\right)\right)\right) \subset A$ e $x_{2}=\pi\left(x_{1}^{+}, \phi\left(x_{1}^{+}\right)\right) \in A \cap M . \quad$ Daí $x_{2}^{+}=I\left(x_{2}\right) \in A$ e $\tilde{\pi}\left(x,\left[\phi(x), \phi(x)+\phi\left(x_{1}^{+}\right)\right]\right) \subset A$. Continuando com este processo segue que $\tilde{\pi}^{+}(x) \subset A$. 
Proposição 2.3.5 Sejam $(X, \pi ; M, I)$ um sistema semidinâmico impulsivo e $A$ um subconjunto fechado de $X$ positivamente $\tilde{\pi}$-invariante. Então $A$ é positivamente $\pi$-invariante.

Demonstração: Suponhamos que $A$ não seja positivamente $\pi$-invariante, isto é, existem $x \in A$ e $\mu \in \mathbb{R}_{+}$tais que $\pi(x, \mu) \notin A$. Definamos

$$
t=\inf \{s>0: \pi(x, s) \notin A\}
$$

Observemos que $t>0$, pois $\tilde{\pi}(x,[0, \phi(x)))=\pi(x,[0, \phi(x))) \subset A$. Como $\pi(x,[0, t)) \subset A$, segue que $\pi(x, t) \in \bar{A}=A$. Assim

$$
\pi(\pi(x, t),[0, \phi(\pi(x, t))))=\tilde{\pi}(\pi(x, t),[0, \phi(\pi(x, t)))) \subset A,
$$

isto é,

$$
\pi(x,[t, t+\phi(\pi(x, t)))) \subset A
$$

e isto contradiz a definição de $t$. Portanto $A$ é positivamente $\pi$-invariante.

Proposição 2.3.6 Sejam $(X, \pi ; M, I)$ um sistema semidinâmico impulsivo e $A$ um subconjunto compacto e positivamente $\widetilde{\pi}$-invariante de $X$. Se $E$ é uma componente conexa de $A I$-invariante, então $E$ é positivamente $\widetilde{\pi}$-invariante.

Demonstração: Seja $x \in E$, então $\tilde{\pi}(x,[0, \phi(x)))=\pi(x,[0, \phi(x))) \subset A$. Pela continuidade de $\pi$ e conexidade de $E$ temos $\pi(x,[0, \phi(x))) \subset E$. Se $\phi(x)=+\infty$ concluímos a prova. Do contrário se $\phi(x)<+\infty$, então $x_{1}=\pi(x, \phi(x)) \in \bar{E}=E$ e $x_{1}^{+}=I\left(x_{1}\right) \in E$, pois $E$ é $I$-invariante. As$\operatorname{sim} \tilde{\pi}\left(x,\left[\phi(x), \phi(x)+\phi\left(x_{1}^{+}\right)\right)\right)=\pi\left(x_{1}^{+},\left[0, \phi\left(x_{1}^{+}\right)\right)\right) \subset E$, se $\phi\left(x_{1}^{+}\right)=+\infty$ concluímos a prova. Do contrário se $\phi\left(x_{1}^{+}\right)<+\infty$, então $x_{2}=\pi\left(x_{1}^{+}, \phi\left(x_{1}^{+}\right)\right) \in \bar{E}=E$ e $x_{2}^{+}=I\left(x_{2}\right) \in E$. Continuando com este processo concluímos que $\tilde{\pi}^{+}(x) \subset E$.

\subsection{Conjuntos limites}

No Capítulo 1, Seção 1.2.1, apresentamos os conjuntos limites para um sistema semidinâmico. Nesta seção, consideramos tais conjuntos no contexto de sistemas semidinâmicos impulsivos. 
Definição 2.4.1 Seja $(X, \pi ; M, I)$ um sistema semidinâmico impulsivo. Para cada $x \in X$ definimos o conjunto limite positivo de $x$ com respeito a $\tilde{\pi}$ pelo conjunto

$$
\begin{gathered}
\widetilde{L}^{+}(x)=\left\{y \in X \text { : existe uma sequência }\left\{t_{n}\right\}_{n \geq 1} \subset \mathbb{R}_{+}\right. \text {tal que } \\
\left.t_{n} \stackrel{n \rightarrow+\infty}{\longrightarrow}+\infty \text { e } \pi\left(x, t_{n}\right) \stackrel{n \rightarrow+\infty}{\longrightarrow} y\right\} .
\end{gathered}
$$

O conjunto prolongado de $x$ com respeito a $\tilde{\pi}$ é definido por

$$
\begin{gathered}
\widetilde{D}^{+}(x)=\left\{y \in X \text { : existem sequências }\left\{x_{n}\right\}_{n \geq 1} \subset X \text { e }\left\{t_{n}\right\}_{n \geq 1} \subset \mathbb{R}_{+}\right. \\
\text {tais que } \left.x_{n} \stackrel{n \rightarrow+\infty}{\longrightarrow} x \text { e } \tilde{\pi}\left(x_{n}, t_{n}\right) \stackrel{n \rightarrow+\infty}{\longrightarrow} y\right\}
\end{gathered}
$$

e o prolongamento do conjunto limite positivo de $x$ com respeito a $\widetilde{\pi}$ é dado por

$$
\begin{gathered}
\widetilde{J}^{+}(x)=\left\{y \in X: \text { existem sequências }\left\{x_{n}\right\}_{n \geq 1} \subset X \text { e }\left\{t_{n}\right\}_{n \geq 1} \subset \mathbb{R}_{+}\right. \\
\text {tais que } \left.x_{n} \stackrel{n \rightarrow+\infty}{\longrightarrow} x, t_{n} \stackrel{n \rightarrow+\infty}{\longrightarrow}+\infty \text { e } \tilde{\pi}\left(x_{n}, t_{n}\right) \stackrel{n \rightarrow+\infty}{\longrightarrow} y\right\}
\end{gathered}
$$

Podemos caracterizar os conjuntos $\widetilde{L}^{+}(x), \widetilde{J}^{+}(x)$ e $\widetilde{D}^{+}(x)$ da seguinte maneira.

Lema 2.4.2 Sejam $(X, \pi ; M, I)$ um sistema semidinâmico impulsivo e $x \in X$. Então

$$
\widetilde{L}^{+}(x)=\bigcap_{t>0} \overline{\tilde{\pi}(x,[t,+\infty))}, \quad \widetilde{D}^{+}(x)=\bigcap_{\varepsilon>0} \overline{\bigcup_{t \geq 0} \tilde{\pi}(B(x, \varepsilon), t)}
$$

e

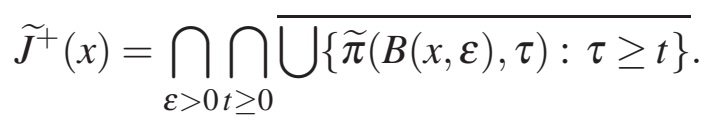

Demonstração: A prova é semelhante as demonstrações dos Lemas 1.2.12 e 1.2.16.

Decorre do Lema 2.4.2 o seguinte resultado.

Proposição 2.4.3 Os conjuntos $\widetilde{L}^{+}(x), \widetilde{J}^{+}(x)$ e $\widetilde{D}^{+}(x), x \in X$, são fechados em $X$. 
Diferente do caso contínuo, o conjunto limite de um ponto $x \in X$ pode não ser positivamente $\tilde{\pi}$-invariante. Mostremos este fato no exemplo seguinte.

Exemplo 2.4.4 Consideremos o sistema impulsivo em $X=\mathbb{R}^{2}$ dado por

$$
\left\{\begin{array}{l}
\dot{x}=x \\
\dot{y}=0 \\
I: M \rightarrow N
\end{array}\right.
$$

onde $M=\left\{(x, y) \in \mathbb{R}^{2}: x^{2}+y^{2}=9\right\}, N=\left\{(x, y) \in \mathbb{R}^{2}: x^{2}+y^{2}=1\right\}$ e a função $I: M \rightarrow N$ é definida da seguinte forma: dado $(x, y) \in M$, consideremos o segmento de reta que liga a origem de $\mathbb{R}^{2}$ ao ponto $(x, y)$. O ponto $I(x, y)$ é definido como sendo o ponto do conjunto $N$ que pertence a interseção deste segmento de reta com o conjunto $N$. A Figura 2.3 descreve a curva integral do sistema impulsivo para o ponto $p=(1,2)$.

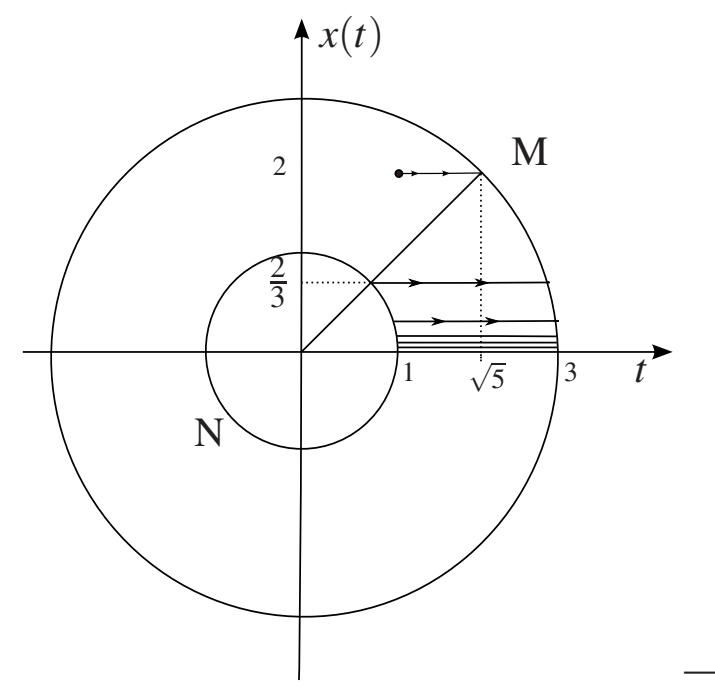

Figura 2.3: Curva integral do sistema impulsivo (2.1), com condição inicial $(0,(1,2))$.

Assim, temos que $\widetilde{L}^{+}(p)=\left\{(x, y) \in \mathbb{R}^{2}: x \in[1,3]\right.$ e $\left.y=0\right\}$. Entretanto, notemos que o ponto $q=(3,0) \in \widetilde{L}^{+}(p)$, porém sua trajetória impulsiva, $\widetilde{\pi}^{+}(q)$, não está contida em $\widetilde{L}^{+}(p)$.

Na sequência, apresentamos um resultado de invariância para o conjunto limite de um ponto em um sistema impulsivo. Para demonstrar esse resultado de invariância, vamos precisar do seguinte lema auxiliar sobre convergência em sistema impulsivos. 
Lema 2.4.5 Sejam $(X, \pi ; M, I)$ um sistema semidinâmico impulsivo e $x \in X \backslash M$. Suponhamos que $I(M) \cap M=\emptyset$. Seja $\left\{z_{n}\right\}_{n \geq 1}$ uma sequência em $X$ tal que $z_{n} \stackrel{n \rightarrow+\infty}{\longrightarrow} x$. Dado $t \geq 0$, existe uma sequência $\left\{\varepsilon_{n}\right\}_{n \geq 1} \subset \mathbb{R}$ tal que $\varepsilon_{n} \stackrel{n \rightarrow+\infty}{\longrightarrow} 0$ e $\tilde{\pi}\left(z_{n}, t+\varepsilon_{n}\right) \stackrel{n \rightarrow+\infty}{\longrightarrow} \tilde{\pi}(x, t)$.

Demonstração: Se $\phi(x)=+\infty$, segue pela continuidade da $\phi$ sobre $X \backslash M$, que dado $t \in[0,+\infty)$ existe um natural $n_{0}$ tal que, para $n \geq n_{0}$, temos $\phi\left(z_{n}\right)>t$. Consequentemente, para $n \geq n_{0}$, $\tilde{\pi}\left(z_{n}, t\right)=\pi\left(z_{n}, t\right)$ e o resultado segue da continuidade de $\pi$.

Agora, suponhamos que $\phi(x)<+\infty$. Então, já que $\phi$ é contínua em $X \backslash M$, podemos assumir que $\phi\left(z_{n}\right)<+\infty$ para todo natural $n$.

Temos três casos a considerar.

Caso 1: Para $0 \leq t<\phi(x)$, seja $\varepsilon<\phi(x)-t$.

Pela continuidade de $\phi$ em $X \backslash M$, existe $n_{0} \in \mathbb{N}$ tal que $\phi(x)-\varepsilon<\phi\left(z_{n}\right)$ para todo $n \geq n_{0}$. Logo $t<\phi\left(z_{n}\right)$ e $\tilde{\pi}\left(z_{n}, t\right)=\pi\left(z_{n}, t\right)$ para $n \geq n_{0}$. Tomando $\varepsilon_{n}=0, n=1,2, \ldots$, segue que

$$
\tilde{\pi}\left(z_{n}, t+\varepsilon_{n}\right)=\pi\left(z_{n}, t\right) \stackrel{n \rightarrow+\infty}{\longrightarrow} \pi(x, t)=\tilde{\pi}(x, t) .
$$

Caso 2: Seja $t=\phi(x)$.

Temos $\tilde{\pi}(x, t)=x_{1}^{+}$. Note que

$$
\left(z_{n}\right)_{1}=\pi\left(z_{n}, \phi\left(z_{n}\right)\right) \stackrel{n \rightarrow+\infty}{\longrightarrow} \pi(x, \phi(x))=x_{1} .
$$

Mas como I é contínua, então

$$
I\left(\left(z_{n}\right)_{1}\right) \stackrel{n \rightarrow+\infty}{\longrightarrow} I\left(x_{1}\right)
$$

isto é, $\left(z_{n}\right)_{1}^{+} \stackrel{n \rightarrow+\infty}{\longrightarrow} x_{1}^{+}$. Pela continuidade de $\phi$ em $X \backslash M$, segue que $\left|\phi\left(z_{n}\right)-\phi(x)\right| \stackrel{n \rightarrow+\infty}{\longrightarrow} 0$. Logo $\phi\left(z_{n}\right)=t+\varepsilon_{n}$, onde $\left\{\varepsilon_{n}\right\}_{n \geq 1}$ é uma sequência de números reais com $\varepsilon_{n} \stackrel{n \rightarrow+\infty}{\longrightarrow} 0$, daí

$$
\tilde{\pi}\left(z_{n}, t+\varepsilon_{n}\right)=\tilde{\pi}\left(z_{n}, \phi\left(z_{n}\right)\right)=\left(z_{n}\right)_{1}^{+} \stackrel{n \rightarrow+\infty}{\longrightarrow} x_{1}^{+}=\tilde{\pi}(x, t) .
$$

Caso 3: Agora, seja $t>\phi(x)$. 
Neste caso, existe $m \in\{1,2,3,4, \ldots\}$ tal que $t=\sum_{i=0}^{m-1} \phi\left(x_{i}^{+}\right)+t^{\prime} \operatorname{com} 0 \leq t^{\prime}<\phi\left(x_{m}^{+}\right)$. Definamos $\left\{\left(z_{n}\right)_{i}\right\}_{i \geq 1}$ indutivamente por

$$
\left(z_{n}\right)_{1}=\pi\left(z_{n}, \phi\left(z_{n}\right)\right) \text { e }\left(z_{n}\right)_{i+1}=\pi\left(\left(z_{n}\right)_{i}^{+}, \phi\left(\left(z_{n}\right)_{i}^{+}\right)\right), \quad i \in\{1,2,3,4, \ldots\} .
$$

Seja $t_{n}=\sum_{i=0}^{m-1} \phi\left(\left(z_{n}\right)_{i}^{+}\right)$. Então, como $\phi\left(z_{n}\right) \stackrel{n \rightarrow+\infty}{\longrightarrow} \phi(x)$, temos

$$
\left(z_{n}\right)_{1}=\pi\left(z_{n}, \phi\left(z_{n}\right)\right) \stackrel{n \rightarrow+\infty}{\longrightarrow} \pi(x, \phi(x))=x_{1} .
$$

Segue da continuidade de $I$ que $I\left(\left(z_{n}\right)_{1}\right) \stackrel{n \rightarrow+\infty}{\longrightarrow} I\left(x_{1}\right)$, isto é, $\left(z_{n}\right)_{1}^{+} \stackrel{n \rightarrow+\infty}{\longrightarrow} x_{1}^{+}$. Como

$$
\phi\left(\left(z_{n}\right)_{1}^{+}\right) \stackrel{n \rightarrow+\infty}{\longrightarrow} \phi\left(x_{1}^{+}\right)
$$

temos

$$
\left(z_{n}\right)_{2}=\pi\left(\left(z_{n}\right)_{1}^{+}, \phi\left(\left(z_{n}\right)_{1}^{+}\right)\right) \stackrel{n \rightarrow+\infty}{\longrightarrow} \pi\left(x_{1}^{+}, \phi\left(x_{1}^{+}\right)\right)=x_{2}
$$

Prosseguindo desta maneira, obtemos

$$
\left(z_{n}\right)_{i} \stackrel{n \rightarrow+\infty}{\longrightarrow} x_{i}
$$

para todo $i=1,2,3 \ldots$. Pela continuidade de $I$, segue que

$$
\left(z_{n}\right)_{i}^{+} \stackrel{n \rightarrow+\infty}{\longrightarrow} x_{i}^{+}
$$

para todo $i=1,2,3, \ldots$ Assim $\sum_{i=0}^{m-1} \phi\left(\left(z_{n}\right)_{i}^{+}\right) \stackrel{n \rightarrow+\infty}{\longrightarrow} \sum_{i=0}^{m-1} \phi\left(x_{i}^{+}\right)$. Então existe uma sequência de números reais $\left\{\varepsilon_{n}\right\}_{n \geq 1}, \operatorname{com} \varepsilon_{n}=\left(t_{n}+t^{\prime}-t\right)$ e $\varepsilon_{n} \stackrel{n \rightarrow+\infty}{\longrightarrow} 0$. Então

$$
\tilde{\pi}\left(z_{n}, t+\varepsilon_{n}\right)=\pi\left(\left(z_{n}\right)_{m}^{+}, t^{\prime}\right) \stackrel{n \rightarrow+\infty}{\longrightarrow} \pi\left(x_{m}^{+}, t^{\prime}\right)=\tilde{\pi}(x, t)
$$

E o lema está provado. 
Teorema 2.4.6 Seja $(X, \pi ; M, I)$ um sistema semidinâmico impulsivo. Suponhamos que $I(M) \cap M=\emptyset$. Então $\widetilde{L}^{+}(x) \backslash M$ é positivamente $\widetilde{\pi}$-invariante para todo $x \in X$.

Demonstração: Sejam $y \in \widetilde{L}^{+}(x) \backslash M$ e $t \geq 0$ arbitrário. Então existe uma sequência $\left\{t_{n}\right\}_{n \geq 1} \subset \mathbb{R}_{+}$com $t_{n} \stackrel{n \rightarrow+\infty}{\longrightarrow}+\infty$ tal que $\tilde{\pi}\left(x, t_{n}\right) \stackrel{n \rightarrow+\infty}{\longrightarrow} y$. Como $y \notin M$, podemos supor que $\left\{\tilde{\pi}\left(x, t_{n}\right)\right\}_{n \geq 1} \subset X \backslash M$. Então pelo Lema 2.4.5, existe uma sequência $\left\{\varepsilon_{n}\right\}_{n \geq 1} \subset \mathbb{R}$ com $\varepsilon_{n} \stackrel{n \rightarrow+\infty}{\longrightarrow} 0$ tal que $\tilde{\pi}\left(x, t_{n}+t+\varepsilon_{n}\right)=\tilde{\pi}\left(\tilde{\pi}\left(x, t_{n}\right), t+\varepsilon_{n}\right) \stackrel{n \rightarrow+\infty}{\longrightarrow} \tilde{\pi}(y, t)$. Notemos que $\left\{t_{n}+t+\varepsilon_{n}\right\}_{n \geq 1} \subset \mathbb{R}_{+}$com $t_{n}+t+\varepsilon_{n} \stackrel{n \rightarrow+\infty}{\longrightarrow}+\infty$. Logo $\tilde{\pi}(y, t) \in \widetilde{L}^{+}(x) \backslash M$ e como $t \geq 0$ foi tomado de maneira arbitrária, o teorema está provado.

Corolário 2.4.7 Sejam $(X, \pi ; M, I)$ um sistema semidinâmico impulsivo e $x \in X$. Suponhamos que $I(M) \cap M=\emptyset$. Se $\widetilde{L}^{+}(x) \cap M=\emptyset$, então $\widetilde{L}^{+}(x)$ é positivamente $\widetilde{\pi}$-invariante.

Para os conjuntos $\widetilde{J}^{+}(x)$ e $\widetilde{D}^{+}(x), x \in X$, temos o seguinte resultado sobre invariância.

Teorema 2.4.8 Seja $(X, \pi ; M, I)$ um sistema semidinâmico impulsivo. Se $I(M) \cap M=\emptyset$, então os conjuntos $\widetilde{D}^{+}(x) \backslash M$ e $\widetilde{J}^{+}(x) \backslash M$ são positivamente $\tilde{\pi}$-invariantes para todo $x \in X$.

Demonstração: A prova é análoga a demonstração do Teorema 2.4.6.

Corolário 2.4.9 Sejam $(X, \pi ; M, I)$ um sistema semidinâmico impulsivo e $x \in X$. Suponhamos que $I(M) \cap M=\emptyset$.

i) Se $\widetilde{J}^{+}(x) \cap M=\emptyset$, então $\widetilde{J}^{+}(x)$ é positivamente $\widetilde{\pi}$-invariante;

ii) Se $\widetilde{D}^{+}(x) \cap M=\emptyset$, então $\widetilde{D}^{+}(x)$ é positivamente $\widetilde{\pi}$-invariante

A seguir, definimos o conceito do conjunto $\widetilde{D}^{+}(A), A \subset X$, que será utilizado nos próximos capítulos.

Definição 2.4.10 Dado $A$ um subconjunto de $X$, definimos $\widetilde{D}^{+}(A)=\cup\left\{\widetilde{D}^{+}(a): a \in A\right\}$. 


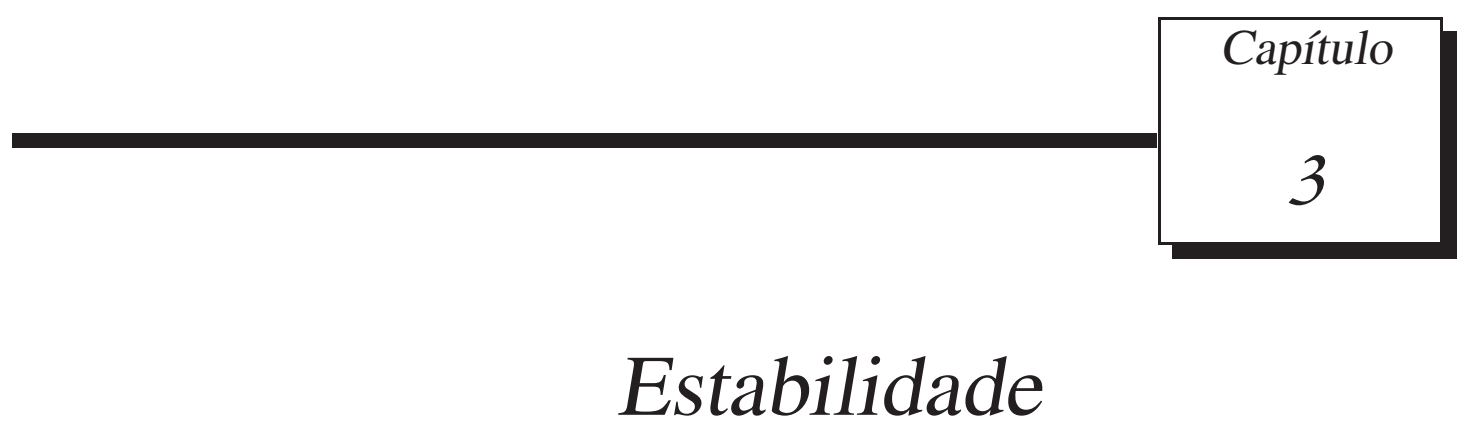

Neste capítulo, apresentamos a teoria de estabilidade para sistemas semidinâmicos impulsivos. Na Seção 3.1, definimos alguns conceitos de estabilidade e apresentamos alguns resultados de estabilidade para conjuntos compactos. Na Seção 3.2, estudamos a estabilidade de componentes conexas de um conjunto compacto. Já na Seção 3.3, apresentamos os conceitos de região de atração e região de atração fraca e exibimos um resultado de estabilidade assintótica para sistemas impulsivos.

As referências utilizadas neste capítulo foram [2], [5] e [14].

\subsection{Estabilidade de sistemas semidinâmicos impulsivos}

Definição 3.1.1 Sejam $(X, \pi ; M, I)$ um sistema semidinâmico impulsivo e $A$ um subconjunto de $X$. Dizemos que o conjunto $A$ é:

a) $\tilde{\pi}$-estável, se dados $\varepsilon>0$ e $x \in A$ existe um $\delta=\delta(x, \varepsilon)>0$ tal que $\tilde{\pi}(B(x, \delta),[0,+\infty)) \subset$ $B(A, \varepsilon)$

b) orbitalmente $\widetilde{\pi}$-estável, se para toda vizinhança $U$ de $A$ existe uma vizinhança $V$ de $A$, positivamente $\widetilde{\pi}$-invariante, tal que $V \subset U$;

c) $\tilde{\pi}$-estável segundo Bhatia e Hajek, se para todo $x \in A$ e $y \notin A$ existem vizinhanças $V$ de $x$ e 
$U$ de $y$ tais que $U \cap \tilde{\pi}(V,[0,+\infty))=\emptyset$

d) equi $\tilde{\pi}$-estável, se para todo $x \notin A$, existe um $\delta=\delta(x)>0$ tal que $x \notin \overline{\widetilde{\pi}(B(A, \delta),[0,+\infty))}$;

e) uniformemente $\tilde{\pi}$-estável, se para todo $\varepsilon>0$, existe um $\delta=\delta(\varepsilon)>0$ tal que $\tilde{\pi}(B(A, \delta),[0,+\infty)) \subset B(A, \varepsilon)$.

Definição 3.1.2 Sejam $(X, \pi ; M, I)$ um sistema semidinâmico impulsivo e $A$ um subconjunto de $X$. Dizemos que o conjunto $A$ é $I$-estável, se para todo $\varepsilon>0$, existe $\delta=\delta(\varepsilon)>0$ tal que $I(M \cap B(A, \delta)) \subset B(A, \varepsilon)$.

O resultado seguinte relaciona o conceito de estabilidade com invariância.

Teorema 3.1.3 Sejam $(X, \pi ; M, I)$ um sistema semidinâmico impulsivo e $A$ um subconjunto de $X$. Se $A$ é um conjunto compacto e $\widetilde{\pi}$-estável então $A$ é positivamente $\widetilde{\pi}$-invariante e positivamente $\pi$-invariante. Além disso, se não existe ponto inicial em $M \cap A$, então $A$ é $I$-invariante.

Demonstração: Primeiramente, mostremos que $A$ é positivamente $\widetilde{\pi}$-invariante. Segue da estabilidade de $A$ que dados $\varepsilon>0$ e $x \in A$, existe $\delta_{x}=\delta(x, \varepsilon)>0$ tal que $\tilde{\pi}\left(B\left(x, \delta_{x}\right),[0,+\infty)\right) \subset B(A, \varepsilon)$. Assim

$$
\tilde{\pi}^{+}(x) \subset B(A, \varepsilon)
$$

para todo $\varepsilon>0$. Portanto,

$$
\tilde{\pi}^{+}(x) \subset \bigcap_{\varepsilon>0} B(A, \varepsilon)=\bar{A}=A
$$

e $A$ é positivamente $\widetilde{\pi}$-invariante.

Pela Proposição 2.3 .5 , temos que $A$ é positivamente $\pi$-invariante.

Mostremos que $A$ é $I$-invariante. Seja $x \in M \cap A$ arbitrário. Então existem $y \in X$ e $t \in \mathbb{R}_{+}$ tais que $\pi(y, t)=x$. Dado $\varepsilon>0$, existe $\delta>0$ tal que $\tilde{\pi}(B(x, \delta),[0,+\infty)) \subset B(A, \varepsilon)$. Assim existe $s \in[0, t)$ tal que $\pi(y, s) \in B(x, \delta)$. Portanto $\tilde{\pi}(\pi(y, s),[0,+\infty)) \subset B(A, \varepsilon) \mathrm{e}$

$$
I(x)=I(\pi(y, t))=I(\pi(\pi(y, s), t-s))=\tilde{\pi}(\pi(y, s), t-s) \in B(A, \varepsilon)
$$

Como $\varepsilon$ é arbitrário, segue que $I(x) \in \bigcap_{\varepsilon>0} B(A, \varepsilon)=\bar{A}=A$. Isto conclui o teorema. 
Observação 3.1.4 Notemos que não precisamos de compacidade na prova da primeira parte do teorema anterior, ou seja, basta que $A$ seja fechado para concluirmos que $A$ é positivamente $\widetilde{\pi}$-invariante.

Os conceitos de $\pi$-estabilidade e $\widetilde{\pi}$-estabilidade não são equivalentes em geral. A seguir, mostramos dois exemplos que ilustram este fato.

Exemplo 3.1.5 Seja $X=\{(r, \theta): r \geq 1\}$ em coordenadas polares. Consideremos o sistema semidinâmico dado pelas equações

$$
\left\{\begin{array}{l}
\dot{r}=\frac{r-1}{100} \\
\dot{\theta}=1
\end{array}\right.
$$

Definamos o conjunto impulsivo e a função impulso por

$$
M=[1,+\infty) \times\left\{\frac{\pi}{2}\right\} \quad \text { e } \quad I(r, \theta)=\left(\frac{r+99}{100}, \frac{3 \pi}{2}\right)
$$

respectivamente.

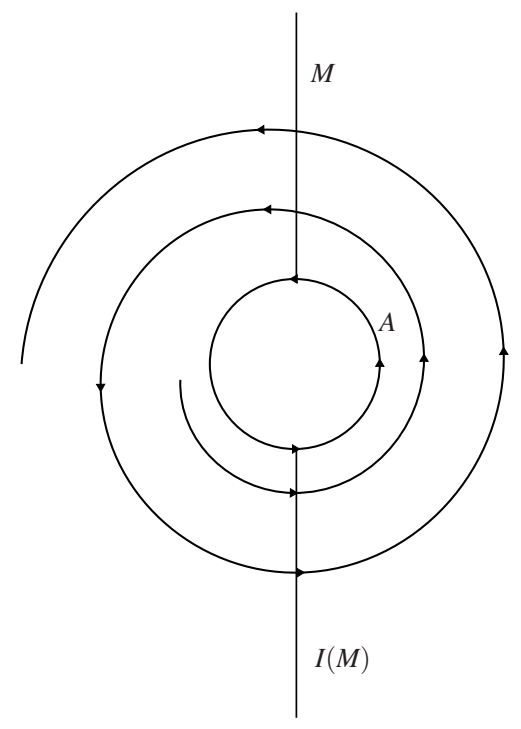

Figura 3.1: Curva integral do sistema impulsivo.

O Conjunto $A=\{(r, \theta): r=1\}$ não é $\pi$-estável já que as trajetórias do sistema vão espiralando do círculo unitário para o infinito, veja Figura 3.1. No entanto $A$ é $\widetilde{\pi}$-estável pois a órbita de um 
ponto que inicia numa vizinhança de $A$ permanece na vizinhança de $A$.

Exemplo 3.1.6 Seja $X=\{(r, \theta): 1 \leq r \leq 2\}$ em coordenadas polares. Consideremos o sistema semidinâmico dado por

$$
\left\{\begin{array}{l}
\dot{r}=0 \\
\dot{\theta}=1
\end{array}\right.
$$

Sejam $M_{1}=\left[1, \frac{3}{2}\right] \times\left\{\frac{\pi}{2}\right\}, M_{2}=[1,2] \times\left\{\frac{3 \pi}{2}\right\}$ e $M=M_{1} \cup M_{2}$. Definamos o operador $I: M \rightarrow X$ por

$$
I\left(r, \frac{\pi}{2}\right)=(2 r-1,0) \quad \text { para } \quad 1 \leq r \leq \frac{3}{2}
$$

e

$$
I\left(r, \frac{3 \pi}{2}\right)=\left(\frac{r+1}{2}, \pi\right) \quad \text { para } \quad 1 \leq r \leq 2 .
$$

O conjunto $A=\{(r, \theta): r=1\}$ é $\pi$-estável e $I$-estável. No entanto, $A$ não é $\widetilde{\pi}$-estável já que

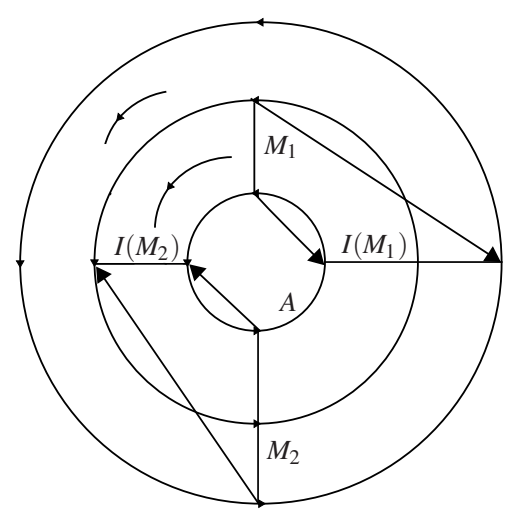

Figura 3.2: Curva integral do sistema impulsivo.

as trajetórias do sistema impulsivo que iniciam em pontos de $\{(r, \theta): 1<r<3 / 2,0<\theta<\pi / 2\}$ passam para o anel $\{(r, \theta): 3 / 2 \leq r \leq 2\}$ depois de um certo tempo. É claro que $A$ é $I$-invariante e positivamente $\pi$-invariante.

Sob algumas condições um sistema $\pi$-estável pode ser $\widetilde{\pi}$-estável. Vejamos o próximo teorema.

Teorema 3.1.7 Sejam $X$ localmente compacto e $A$ um subconjunto compacto e $\pi$-estável de $X$. Suponhamos que exista um $v>0$ tal que, para todo $x \in B(A, v)$, as seguintes condições são válidas: 
i) se $x \in M$, então $\rho(I(x), A) \leq \rho(x, A)$;

ii) se $x \in I(M)$ e $\phi(x)<+\infty$, então $\rho(\pi(x, \phi(x)), A) \leq \rho(x, A)$.

Então $A$ é $\tilde{\pi}$-estável.

Demonstração: Seja $\varepsilon>0$. Podemos supor que $\varepsilon<v$. Pela compacidade de $A$ é suficiente mostramos que existe $\delta>0$ tal que $\tilde{\pi}^{+}(B(A, \delta)) \subset B(A, \varepsilon)$.

Como $A$ é compacto e $\pi$-estável existe $\eta>0, \eta<\varepsilon$, tal que $\pi^{+}(x) \subset B(A, \varepsilon)$ para todo $x \in B(A, \eta)$. De mesmo modo, existe $\delta>0$ tal que $\pi^{+}(x) \subset B(A, \eta / 2)$ para todo $x \in B(A, \delta)$ $(\delta<\eta / 2)$.

Vamos mostrar que $\tilde{\pi}^{+}(x) \subset B(A, \varepsilon)$ para todo $x \in B(A, \delta)$. De fato, seja $x \in B(A, \delta)$. Se $\phi(x)=+\infty$, então $\tilde{\pi}^{+}(x)=\pi^{+}(x) \subset B(A, \varepsilon)$. Assumamos que $\phi\left(x_{j}^{+}\right)<+\infty$ para todo $j=0,1,2, \ldots$ Então

$$
\tilde{\pi}(x,[0, \phi(x)))=\pi(x,[0, \phi(x))) \subset B(A, \eta / 2) \subset B(A, \varepsilon) \subset B(A, v)
$$

$\operatorname{Logo} x_{1}=\pi(x, \phi(x)) \in \overline{B(A, \eta / 2)}$, ou seja,

$$
\rho\left(x_{1}, A\right) \leq \eta / 2<\varepsilon<v .
$$

Pelo item $i$, concluímos $\rho\left(x_{1}^{+}, A\right)=\rho\left(I\left(x_{1}\right), A\right) \leq \rho\left(x_{1}, A\right) \leq \eta / 2<\eta$ e portanto

$$
\pi\left(x_{1}^{+},\left[0, \phi\left(x_{1}^{+}\right)\right)\right)=\tilde{\pi}\left(x,\left[\phi(x), \phi(x)+\phi\left(x_{1}^{+}\right)\right)\right) \subset B(A, \varepsilon) .
$$

Pelo item $i i)$, obtemos

$$
\rho\left(x_{2}, A\right)=\rho\left(\pi\left(x_{1}^{+}, \phi\left(x_{1}^{+}\right)\right), A\right) \leq \rho\left(x_{1}^{+}, A\right) \leq \eta / 2 .
$$

Por $i$ ) segue que

$$
\rho\left(I\left(x_{2}\right), A\right)=\rho\left(x_{2}^{+}, A\right) \leq \rho\left(x_{2}, A\right) \leq \eta / 2<\eta .
$$

Continuando com este processo obtemos $\rho\left(x_{n}, A\right) \leq \eta / 2, \rho\left(x_{n}^{+}, A\right) \leq \eta / 2$ e $\pi\left(x_{n}^{+},\left[0, \phi\left(x_{n}^{+}\right)\right)\right) \subset$ $B(A, \varepsilon)$. Portanto $\tilde{\pi}^{+}(x) \subset B(A, \varepsilon)$. O resultado está provado 
Proposição 3.1.8 Sejam $(X, \pi ; M, I)$ um sistema semidinâmico impulsivo e $A$ um subconjunto compacto de $X$. Se $A$ é $\pi$-estável e $A \cap M=\emptyset$ então $A$ é $\tilde{\pi}$-estável.

Demonstração: Como $A$ é compacto e $M$ é fechado, existe $\beta>0$ tal que $B(A, \beta) \cap M=\emptyset$. Da$\operatorname{dos} \varepsilon>0, \varepsilon<\beta$, e $x \in A$ existe $\delta>0$ tal que $\pi^{+}(B(x, \delta)) \subset B(A, \varepsilon)$. Portanto $\tilde{\pi}^{+}(B(x, \delta))=$ $\pi^{+}(B(x, \delta)) \subset B(A, \varepsilon)$.

Teorema 3.1.9 Sejam $(X, \pi ; M, I)$ um sistema semidinâmico impulsivo e $A$ um subconjunto compacto de $X$. Suponhamos que $A$ seja $\pi$-estável e que exista $v>0$ tal que $I(M \cap B(A, v)) \subset A$. Então $A$ é $\widetilde{\pi}$-estável.

Demonstração: Dado $\varepsilon>0$, existe $\delta>0$ tal que $\pi^{+}(B(A, \delta)) \subset B(A, \varepsilon)$, pois $A$ é compacto. Podemos assumir que $\varepsilon<v$. Mostraremos que $\tilde{\pi}^{+}(B(A, \delta)) \subset B(A, \varepsilon)$. Seja $x \in B(A, \delta)$ então

$$
\tilde{\pi}(x,[0, \phi(x)))=\pi(x,[0, \phi(x))) \subset B(A, \varepsilon) \subset B(A, v) .
$$

Se $\phi(x)=+\infty$, o resultado segue. Assumamos que $\phi\left(x_{j}^{+}\right)<+\infty$ para todo $j=0,1,2, \ldots$ Então

$$
x_{1}=\pi(x, \phi(x)) \in \overline{B(A, \varepsilon)} \subset B(A, v) \cap M
$$

e por hipótese $I\left(x_{1}\right)=x_{1}^{+} \in A$. Logo

$$
\tilde{\pi}\left(x_{1}^{+},\left[0, \phi\left(x_{1}^{+}\right)\right)\right)=\pi\left(x_{1}^{+},\left[0, \phi\left(x_{1}^{+}\right)\right)\right) \subset B(A, \varepsilon),
$$

e consequentemente $x_{2}=\pi\left(x_{1}^{+}, \phi\left(x_{1}^{+}\right)\right) \in B(A, v) \cap M$ e $I\left(x_{2}\right)=x_{2}^{+} \in A$. Continuando com este processo, segue que $\tilde{\pi}^{+}(x) \subset B(A, \varepsilon)$ para todo $x \in B(A, \delta)$.

Seja $X$ um espaço localmente compacto. Os conceitos de estabilidade, estabilidade orbital e estabilidade no sentido de Bhatia e Hajek são equivalentes para conjuntos compactos em $X$. Além disso, um conjunto compacto $A \subset X$ é estável se, e somente se, $D^{+}(A)=A$. Este resultado é chamado de Teorema de Ura. A prova para o caso contínuo pode ser encontrada em [2]. A seguir, apresentamos a generalização deste resultado para o caso impulsivo. 
Teorema 3.1.10 Sejam $(X, \pi ; M, I)$ um sistema semidinâmico impulsivo e $A$ um subconjunto compacto de $X$. Suponhamos que $X$ seja localmente compacto. Então as seguintes condições são equivalentes:

i) $A$ é $\widetilde{\pi}$-estável;

ii) A é orbitalmente $\widetilde{\pi}$-estável;

iii) A é $\widetilde{\pi}$-estável segundo Bhatia e Hajek;

iv) $\widetilde{D}^{+}(A)=A$.

Demonstração: $i) \Rightarrow$ ii) Seja $U$ uma vizinhança de $A$. Como $A$ é compacto existe uma vizinhança $V$ de $A$ tal que $V \subset \tilde{\pi}^{+}(V) \subset U$. Notemos que $\tilde{\pi}^{+}(V)$ é uma vizinhança de $A$ positivamente $\widetilde{\pi}$-invariante e contém $V$.

ii) $\Rightarrow$ iii) Sejam $x \in A$ e $y \notin A$. Então existem abertos $W$ e $V$ tais que $A \subset W, y \in V$ e $W \cap V=\emptyset$. Por hipótese, existe uma vizinhança positivamente $\widetilde{\pi}$-invariante $U$ de $A$, tal que $U \subset W$. Então $U$ e $V$ satisfazem as condições da definição de estabilidade segundo Bathia e Hajek.

iii) $\Rightarrow$ iv) É claro que $A \subset \widetilde{D}^{+}(A)$. Mostremos que $\widetilde{D}^{+}(A) \subset A$. Sejam $x \in A$ e $y \notin A$, então existem vizinhanças $U$ e $V$ de $x$ e $y$, respectivamente, tais que

$$
V \cap \tilde{\pi}^{+}(U)=\emptyset
$$

Consideremos as sequências $\left\{x_{n}\right\}_{n \geq 1} \subset X$ com $x_{n} \stackrel{n \rightarrow+\infty}{\longrightarrow} x$ e $\left\{t_{n}\right\}_{n \geq 1} \subset \mathbb{R}_{+}$. Mostraremos que $y \notin \widetilde{D}^{+}(A)$. Ora, para a vizinhança $U$ existe $n_{0} \in \mathbb{N}$ tal que

$$
x_{n} \in U \quad \text { e } \quad \tilde{\pi}\left(x_{n}, t_{n}\right) \in \tilde{\pi}^{+}(U)
$$

para todo $n>n_{0}$. No entanto, $\tilde{\pi}\left(x_{n}, t_{n}\right) \notin V$ pois $V \cap \widetilde{\pi}^{+}(U)=\emptyset$. Portanto a sequência $\left\{\tilde{\pi}\left(x_{n}, t_{n}\right)\right\}_{n \geq 1}$ não converge para $y$. Desta forma, se $y \notin A$ então $y \notin \widetilde{D}^{+}(A)$, como queríamos demonstrar. Portanto $\widetilde{D}^{+}(A)=A$.

$i v) \Rightarrow i$ ) Vamos supor que $A$ não seja $\widetilde{\pi}$-estável, ou seja, que existem $\varepsilon>0, x \in A$ e sequências 
$\left\{x_{n}\right\}_{n \geq 1} \subset X$ e $\left\{t_{n}\right\}_{n \geq 1} \subset \mathbb{R}_{+}$tais que $x_{n} \stackrel{n \rightarrow+\infty}{\longrightarrow} x \mathrm{e}$

$$
\tilde{\pi}\left(x_{n}, t_{n}\right) \notin \overline{B(A, \varepsilon)},
$$

para cada $n \in \mathbb{N}^{*}$. Podemos supor que $\overline{B(A, \varepsilon)}$ seja compacto e que $\left\{x_{n}\right\}_{n \geq 1} \subset B(A, \varepsilon)$. Primeiro, suponhamos que existe uma subsequência $\left\{x_{n_{k}}\right\}_{k \geq 1}$ de $\left\{x_{n}\right\}_{n \geq 1}$ tal que $t_{n_{k}}<\phi\left(x_{n_{k}}\right)$ para todo $k \in \mathbb{N}^{*}$. Então $\tilde{\pi}\left(x_{n_{k}},\left[0, t_{n_{k}}\right]\right)=\pi\left(x_{n_{k}},\left[0, t_{n_{k}}\right]\right)$. Segue da continuidade da função $\pi$ que existe $s_{n_{k}} \in\left[0, t_{n_{k}}\right]$ tal que $\tilde{\pi}\left(x_{n_{k}}, s_{n_{k}}\right) \in \partial \overline{B(A, \varepsilon)}$, para cada $k \in \mathbb{N}^{*}$. Como $\partial \overline{B(A, \varepsilon)}$ é compacto podemos assumir que $\tilde{\pi}\left(x_{n_{k}}, s_{n_{k}}\right) \stackrel{k \rightarrow+\infty}{\longrightarrow} y \in \partial \overline{B(A, \varepsilon)}$. Mas isso é uma contradição pois $y \in \widetilde{D}^{+}(A)=A$.

Agora, suponhamos que existe uma subsequência $\left\{x_{n_{k}}\right\}_{k \geq 1}$ de $\left\{x_{n}\right\}_{n \geq 1}$ tal que $t_{n_{k}} \geq \phi\left(x_{n_{k}}\right)$ para todo $k \in \mathbb{N}^{*}$. Consideremos o conjunto

$$
\Delta_{n_{k}}=\left\{s \geq 0: \widetilde{\pi}\left(x_{n_{k}}, s\right) \in B(A, \varepsilon)\right\}
$$

$k \in \mathbb{N}^{*}$. Notemos que $0 \in \Delta_{n_{k}}$ para todo $k$. Então definamos

$$
s_{n_{k}}=\inf \left\{t \geq 0: t \in[0, T(x)) \backslash \Delta_{n_{k}}\right\},
$$

$k \in \mathbb{N}^{*}$. Notemos que $\tilde{\pi}\left(x_{n_{k}}, s_{n_{k}}\right) \notin B(A, \varepsilon)$ para todo $k \in \mathbb{N}^{*}$, pois $\tilde{\pi}\left(x_{n_{k}}, \cdot\right)$ é contínua à direita.

Afirmação: Dado $\delta \in(0, \varepsilon)$ existe $n_{0} \in \mathbb{N}$ tal que $\tilde{\pi}\left(x_{n_{k}},\left[0, s_{n_{k}}\right)\right) \subset B(A, \delta)$ para $n_{n_{k}}>n_{0}$.

De fato, suponhamos que a afirmação seja falsa, ou seja, que para todo $k \in \mathbb{N}$ podemos encontrar um $u_{n_{k}} \in\left[0, s_{n_{k}}\right)$ tal que $\tilde{\pi}\left(x_{n_{k}}, u_{n_{k}}\right) \in \overline{\mathscr{A}(A, \delta, \varepsilon)}$. Pela compacidade de $\overline{\mathscr{A}(A, \delta, \varepsilon)}$, podemos assumir que $\tilde{\pi}\left(x_{n_{k}}, u_{n_{k}}\right) \stackrel{k \rightarrow+\infty}{\longrightarrow} y \in \overline{\mathscr{A}(A, \delta, \varepsilon)}$, o que absurdo pois $y \in \widetilde{D}^{+}(A)=A$.

Assim, fixado $n_{k}>n_{0}$, temos

$$
\tilde{\pi}\left(x_{n_{k}},\left[0, s_{n_{k}}\right)\right) \subset B(A, \delta) \quad \text { e } \quad \tilde{\pi}\left(x_{n_{k}}, s_{n_{k}}\right) \notin B(A, \varepsilon) .
$$

Isto mostra que $\tilde{\pi}\left(x_{n_{k}},.\right)$ não é contínua em $s_{n_{k}}$, ou seja, $\widetilde{\pi}\left(x_{n_{k}}, s_{n_{k}}\right)=I\left(y_{n_{k}}\right)$ para algum $y_{n_{k}} \in M$. Além disso, como $\widetilde{\pi}\left(x_{n_{k}},\left[0, s_{n_{k}}\right)\right) \subset B(A, \delta)$, segue que $y_{n_{k}} \in \overline{B(A, \delta)}$. 
Pela compacidade de $\overline{B(A, \delta)}$, podemos assumir que

$$
y_{n_{k}} \stackrel{k \rightarrow+\infty}{\longrightarrow} y \in \overline{B(A, \delta)} \cap M .
$$

Agora, segue da continuidade de $I$ que

$$
I\left(y_{n_{k}}\right) \stackrel{k \rightarrow+\infty}{\longrightarrow} I(y)
$$

Então $I(y) \notin A$, pois $I\left(y_{n_{k}}\right) \notin B(A, \varepsilon)$ para todo $n_{k}>n_{0}$. Por outro lado, $I\left(y_{n_{k}}\right)=\tilde{\pi}\left(x_{n_{k}}, s_{n_{k}}\right)$ com $x_{n_{k}} \stackrel{k \rightarrow+\infty}{\longrightarrow} x$ e $s_{n_{k}} \geq 0$, ou seja, $I(x) \in \widetilde{D}^{+}(x) \subset \widetilde{D}^{+}(A)=A$. Contradição.

Portanto, o teorema está provado.

O próximo resultado mostra a equivalência entre a equi estabilidade e a estabilidade uniforme de conjuntos compactos.

Teorema 3.1.11 Sejam $(X, \pi ; M, I)$ um sistema semidinâmico impulsivo e $A$ um subconjunto compacto de $X$. Suponhamos que $X$ seja localmente. Então $A$ é $\widetilde{\pi}$-estável se, e somente, se $A$ é uniformemente $\tilde{\pi}$-estável.

Demonstração: Seja $A \tilde{\pi}$-estável. Dado $\varepsilon>0$ e $x \in A$ existe $\delta_{x}=\delta(x, \varepsilon)>0$ tal que

$$
\tilde{\pi}\left(B\left(x, \delta_{x}\right),[0,+\infty)\right) \subset B(A, \varepsilon) .
$$

Notemos que $A \subset \bigcup_{x \in A} B\left(x, \delta_{x}\right)$. Mas como $A$ é compacto existem $\left\{x_{1}, x_{2}, \ldots, x_{n}\right\} \subset A$ tais que $A \subset \bigcup_{i=1}^{n} B\left(x, \delta_{x_{i}}\right)$. Seja $\delta=\min \left\{\delta_{x_{i}}: i=1,2, \ldots, n\right\}$, então concluímos que $\tilde{\pi}(B(A, \delta),[0,+\infty)) \subset$ $B(A, \varepsilon)$. A recíproca é imediata.

Observação 3.1.12 O Teorema 3.1.11 continua válido se substituirmos a hipótese de equi $\tilde{\pi}-$ estabilidade por $\tilde{\pi}$-estabilidade. A prova deste fato é igual a prova do caso contínuo e pode ser encontrada em [2] na Proposição 4.2.

De acordo com os Teoremas 3.1.10 e 3.1.11, obtemos o seguinte resultado. 
Teorema 3.1.13 Sejam $(X, \pi ; M, I)$ um sistema semidinâmico impulsivo e $A$ um subconjunto compacto de $X$. Suponhamos que $X$ seja localmente compacto. Então são equivalentes:

i) $A$ é $\widetilde{\pi}$-estável;

ii) A é orbitalmente $\widetilde{\pi}$-estável;

iii) A é $\widetilde{\pi}$-estável segundo Bhatia e Hajek;

iv) A é uniformemente $\widetilde{\pi}$-estável;

v) $A$ é equi $\widetilde{\pi}$-estável;

vi) $\widetilde{D}^{+}(A)=A$.

\subsection{Estabilidade de componentes}

Em sistemas semidinâmicos contínuos $(X, \pi)$, um subconjunto compacto $A$ de $X$ é $\pi$-estável se, e somente se, cada componente conexa de $A$ é $\pi$-estável. Isso não é verdade para o caso impulsivo, o exemplo a seguir ilustra este fato.

Exemplo 3.2.1 Consideremos o sistema semidinâmico em $X=\mathbb{R}^{2}$ dado por

$$
\left\{\begin{array}{l}
\dot{x}=-y \\
\dot{y}=x \\
I: M \rightarrow N
\end{array}\right.
$$

onde $M=\{(0,-2),(0,-1)\}$ e $I((0,-2))=(0,1)$ e $I((0,-1))=(0,2)$. Consideremos os conjuntos $A_{1}=\left\{(x, y) \in \mathbb{R}^{2}: x^{2}+y^{2}=1\right\}, A_{2}=\left\{(x, y) \in \mathbb{R}^{2}: x^{2}+y^{2}=4\right\}$ e $A=A_{1} \cup A_{2}$. O conjunto $A$ é $\tilde{\pi}$-estável, no entanto nenhuma das componentes conexas $A_{1}$ e $A_{2}$ de $A$ são $\widetilde{\pi}$-estáveis.

Proposição 3.2.2 Sejam $(X, \pi ; M, I)$ um sistema semidinâmico e $A$ um subconjunto compacto de $X$. Se qualquer componente conexa de $A$ é $\widetilde{\pi}$-estável, então $A$ é $\widetilde{\pi}$-estável. 
Demonstração: Sejam $\varepsilon>0$ e $x \in A$. Consideremos $A_{x}$ uma componente conexa de $A$ tal que $x \in A_{x}$. Notemos que $B(A, \varepsilon)$ é uma vizinhança de $A_{x}$. Então existe $\delta>0$ tal que $\tilde{\pi}^{+}(B(x, \delta)) \subset$ $B(A, \varepsilon)$. E a proposição está provada.

O Teorema a seguir fornece condições para que a recíproca da proposição anterior seja válida. Lembremos que uma componente $E$ de um conjunto $A \subset X$ é isolada se existem abertos $U$ e $V$ em $X$ tais que $E \subset U, A \backslash E \subset V$ e $V \cap U=\emptyset$.

Teorema 3.2.3 Sejam $(X, \pi ; M, I)$ um sistema semidinâmico e $A$ um subconjunto compacto de $X$. Suponhamos que $X$ seja localmente compacto e $A$ seja $\tilde{\pi}$-estável. Se uma componente conexa $E$ de $A$ é $I$-invariante e isolada, então $E$ é $\widetilde{\pi}$-estável.

Demonstração: Suponhamos que $E$ não seja $\tilde{\pi}$-estável. Então existem $x \in E, \varepsilon>0$ e uma sequência $\left\{x_{n}\right\}_{n \geq 1} \subset X \operatorname{com} x_{n} \stackrel{n \rightarrow+\infty}{\longrightarrow} x$ tais que

$$
\tilde{\pi}^{+}\left(x_{n}\right) \nsubseteq B(E, \varepsilon)
$$

para todo $n \in \mathbb{N}^{*}$. Podemos assumir que $\overline{B(E, \varepsilon)}$ seja compacto e que $\left\{x_{n}\right\}_{n \geq 1} \subset B(A, \varepsilon)$. Como $E$ é isolada, existe uma vizinhança aberta $V$ de $A \backslash E$, a qual podemos supor, sem perda de generalidade, que

$$
B(E, \varepsilon) \cap \bar{V}=\emptyset .
$$

Agora, notemos que $M^{+}\left(x_{n}\right)=\left(\pi^{+}\left(x_{n}\right) \cap M\right) \backslash\left\{x_{n}\right\} \neq \emptyset$ para uma infinidade de naturais $n$. De fato, se isso não ocorrer então $M^{+}\left(x_{n}\right)=\emptyset$ para uma infinidade de naturais $n$. Ou seja, existe uma subsequência $\left\{n_{k}\right\}_{k \geq 1}$ de números naturais tal que $M^{+}\left(x_{n_{k}}\right)=\emptyset$ para todo $k \in \mathbb{N}^{*}$. Logo $x_{n_{k}} \in B(E, \varepsilon)$ e $\tilde{\pi}^{+}\left(x_{n_{k}}\right)=\pi^{+}\left(x_{n_{k}}\right) \nsubseteq B(E, \varepsilon)$ para todo $k \in \mathbb{N}^{*}$. Assim existe $u_{n_{k}} \in \mathbb{R}_{+}$tal que $\pi\left(x_{n_{k}}, u_{n_{k}}\right) \in \partial B(E, \varepsilon)$. Como $\partial B(E, \varepsilon)$ é compacto, podemos assumir que

$$
\pi\left(x_{n_{k}}, u_{n_{k}}\right) \stackrel{k \rightarrow+\infty}{\longrightarrow} y \in \partial B(E, \varepsilon) .
$$

Assim $y \notin A$. Por outro lado, como $x_{n_{k}} \stackrel{k \rightarrow+\infty}{\longrightarrow} x$ e $A$ é $\tilde{\pi}$-estável, segue do Teorema 3.1 .10 que $y \in \widetilde{D}^{+}(x) \subset \widetilde{D}^{+}(A)=A$. Contradição. 
Vamos assumir que $M^{+}\left(x_{n}\right) \neq \emptyset$ para todo $n \in \mathbb{N}$.

Como $B(E, \varepsilon) \cup V$ é uma vizinhança de $A$ e $A$ é $\widetilde{\pi}$-estável, existe uma vizinhança $G_{x}$ de $x$ tal que $\tilde{\pi}^{+}\left(G_{x}\right) \subset B(E, \varepsilon) \cup V$. Seja $n_{0} \in \mathbb{N}$ tal que $x_{n} \in G_{x}$ para $n \geq n_{0}$. Então $\tilde{\pi}^{+}\left(x_{n}\right) \subset B(E, \varepsilon) \cup V$ para todo $n \geq n_{0}$. Segue de (3.1) que o conjunto $\left\{t \in \mathbb{R}_{+}: \tilde{\pi}\left(x_{n}, t\right) \in V\right\} \neq \emptyset$ para todo $n \in \mathbb{N}^{*}$. Desta forma, definamos

$$
t_{n}=\inf \left\{t \in \mathbb{R}_{+}: \tilde{\pi}\left(x_{n}, t\right) \in V\right\}
$$

$n \in \mathbb{N}^{*}$. Então $\tilde{\pi}\left(x_{n},\left[0, t_{n}\right)\right) \subset B(E, \varepsilon)$ e $\tilde{\pi}\left(x_{n}, t_{n}\right) \in V, n \in \mathbb{N}^{*}$. Como $B(E, \varepsilon) \cap \bar{V}=\emptyset$, existe $q_{n} \in \overline{B(E, \varepsilon)}$ tal que

$$
\tilde{\pi}\left(x_{n}, t_{n}\right)=I\left(q_{n}\right)
$$

para $n \in \mathbb{N}^{*}$. Além disso, para cada $n \in \mathbb{N}^{*}$, existe $v_{n}<t_{n}$ tal que

$$
\tilde{\pi}\left(x_{n},\left(v_{n}, t_{n}\right)\right) \cap M=\emptyset .
$$

Consideremos a sequência $\left\{s_{n}\right\}_{n \geq 1} \subset \mathbb{R}_{+}$tal que $s_{n} \in\left(v_{n}, t_{n}\right)$ para todo $n \in \mathbb{N}$ e $t_{n}-s_{n} \stackrel{n \rightarrow+\infty}{\longrightarrow} 0$. Daí, definamos

$$
p_{n}=\tilde{\pi}\left(x_{n}, s_{n}\right) \in \overline{B(E, \varepsilon)} .
$$

Podemos assumir que $p_{n} \stackrel{n \rightarrow+\infty}{\longrightarrow} p \in \overline{B(E, \varepsilon)}$. Logo,

$$
q_{n}=\pi\left(p_{n}, t_{n}-s_{n}\right) \stackrel{n \rightarrow+\infty}{\longrightarrow} \pi(p, 0)=p \in M
$$

pois $q_{n} \in M$ para todo $n \in \mathbb{N}^{*}$ e $M$ é fechado, e pela continuidade de $I$ temos

$$
I\left(q_{n}\right) \stackrel{n \rightarrow+\infty}{\longrightarrow} I(p) \in \bar{V}
$$

pois $I\left(q_{n}\right) \in V$ para todo $n \in \mathbb{N}^{*}$.

Por outro lado, como $p_{n}=\tilde{\pi}\left(x_{n}, s_{n}\right) \stackrel{n \rightarrow+\infty}{\longrightarrow} p$ temos que $p \in \widetilde{D}^{+}(x) \subset \widetilde{D}^{+}(A)=A$. Como $p \in$ $\overline{B(E, \varepsilon)}$, segue que $p \in E$ e como $E$ é $I$-invariante temos $I(p) \in E$. Mas por (3.2) e (3.4) temos uma contradição. 
Portanto $E$ é $\tilde{\pi}$-estável.

Corolário 3.2.4 Seja $X$ localmente compacto e seja $A$ um subconjunto compacto de $X$. Suponhamos que $A$ seja um conjunto $\tilde{\pi}$-estável com um número finito de componentes conexas. Então qualquer componente conexa $I$-invariante de $A$ é $\tilde{\pi}$-estável.

\subsection{Estabilidade assintótica}

Nesta seção, apresentamos o conceito de estabilidade assintótica para sistemas semidinâmicos com impulsos. Iniciamos apresentando os conceitos de região de atração fraca e região de atração para um conjunto.

Definição 3.3.1 Sejam $(X, \pi ; M, I)$ um sistema semidinâmico impulsivo e $A$ um subconjunto de $X$. A região de atração fraca de $A$ com respeito a $\tilde{\pi}$ é dada por

$$
\begin{gathered}
\widetilde{P}_{w}^{+}(A)=\left\{x \in X: \text { para toda vizinhança } U \text { de } A \text { existe uma sequência }\left\{t_{n}\right\}_{n \geq 1} \subset \mathbb{R}_{+}\right. \\
\text {tal que } \left.t_{n} \stackrel{n \rightarrow+\infty}{\longrightarrow}+\infty \quad \text { e } \tilde{\pi}\left(x, t_{n}\right) \in U \text { para todo } n=1,2, \ldots\right\}
\end{gathered}
$$

e a região de atração de $A$ com respeito a $\widetilde{\pi}$ é definida por

$$
\widetilde{P}^{+}(A)=\left\{x \in X: \text { para toda vizinhança } U \text { de } A \text { existe } \tau \in \mathbb{R}_{+} \text {tal que } \tilde{\pi}(x,[\tau,+\infty)) \subset U\right\}
$$

Se $x \in \widetilde{P}_{w}^{+}(A)$ dizemos que $x$ é fracamente $\tilde{\pi}$-atraído para $A$ e se $x \in \widetilde{P}^{+}(A)$ dizemos que $x$ é $\widetilde{\pi}$-atraído para $A$.

Proposição 3.3.2 Sejam $(X, \pi ; M, I)$ um sistema semidinâmico impulsivo e $A$ um subconjunto de $X$. Então:

i) $\widetilde{P}^{+}(A) \subset \widetilde{P}_{w}^{+}(A)$;

ii) $\widetilde{P}^{+}(A)$ e $\widetilde{P}_{w}^{+}(A)$ são positivamente $\widetilde{\pi}$-invariantes. 


\section{Demonstração:}

i) Segue pela definição.

ii) Primeiro, mostremos que $\widetilde{P}^{+}(A)$ é positivamente $\widetilde{\pi}$-invariante. Sejam $x \in \widetilde{P}^{+}(A)$ e $t \geq 0$. Então, dada uma vizinhança $U$ de $A$ existe $\tau \in \mathbb{R}_{+}$tal que $\tilde{\pi}(x,[\tau,+\infty)) \subset U$. Notemos que

$$
\tilde{\pi}(\tilde{\pi}(x, t),[\tau,+\infty))=\tilde{\pi}(x,[\tau+t,+\infty)) \subset \widetilde{\pi}(x,[\tau,+\infty)) \subset U .
$$

Portanto $\widetilde{\pi}(x, t) \in \widetilde{P}^{+}(A)$. Como $t \geq 0$ é arbitrário, segue que $\widetilde{P}^{+}(A)$ é positivamente $\widetilde{\pi}$-invariante.

Provemos agora que $\widetilde{P}_{w}^{+}(A)$ é positivamente $\widetilde{\pi}$-invariante. Sejam $x \in \widetilde{P}_{w}^{+}(A)$ e $t \geq 0$. Então, dada uma vizinhança $U$ de $A$ existe uma sequência $\left\{t_{n}\right\}_{n \geq 1} \subset \mathbb{R}_{+}$tal que $t_{n} \stackrel{n \rightarrow+\infty}{\longrightarrow}+\infty$ e $\tilde{\pi}\left(x, t_{n}\right) \in U$ para todo $n \in \mathbb{N}$. Notemos que existe $n_{0} \in \mathbb{N}$ tal que $t_{n}>t$ para $n>n_{0}$. Assim

$$
\tilde{\pi}\left(\tilde{\pi}(x, t), t_{n}-t\right)=\tilde{\pi}\left(x, t_{n}\right) \in U
$$

para todo $n>n_{0}$. Como $t_{n}-t \stackrel{n \rightarrow+\infty}{\longrightarrow}+\infty$, segue que $\tilde{\pi}(x, t) \in \widetilde{P}_{w}^{+}(A)$. Portanto $\widetilde{P}_{w}^{+}(A)$ é positivamente $\tilde{\pi}$-invariante.

A proposição seguinte apresenta condições necessárias para que um ponto $x \in X$ pertença à região fraca de atração.

Proposição 3.3.3 Sejam $(X, \pi ; M, I)$ um sistema semidinâmico impulsivo, $A$ um subconjunto de $X$ e $x \in A$. Suponhamos que $A \cap \widetilde{L}^{+}(x) \neq \emptyset$ ou exista uma sequência $\left\{t_{n}\right\}_{n \geq 1} \subset \mathbb{R}_{+}$com $t_{n} \stackrel{n \rightarrow+\infty}{\longrightarrow}+\infty$ tal que $\widetilde{\pi}\left(x, t_{n}\right) \in A$. Então $x \in \widetilde{P}_{w}^{+}(A)$.

Demonstração: Suponhamos que $x \notin \widetilde{P}_{w}^{+}(A)$. Então existem uma vizinhança $U$ de $A$ e $\tau \in \mathbb{R}_{+}$tais que

$$
\tilde{\pi}(x,[\tau,+\infty)) \subset X \backslash U
$$

Dada uma sequência $\left\{t_{n}\right\}_{n \geq 1} \subset \mathbb{R}_{+} \operatorname{com} t_{n} \stackrel{n \rightarrow+\infty}{\longrightarrow}+\infty$, podemos obter $n_{0} \in \mathbb{N}$ tal que $t_{n}>\tau$ para 
$n>n_{0} . \log 0$

$$
\widetilde{\pi}\left(x, t_{n}\right) \notin A,
$$

para todo $n>n_{0}$. Por outro lado,

$$
\widetilde{L}^{+}(x)=\bigcap_{t>0} \overline{\tilde{\pi}(x,[t,+\infty))} \subset \overline{X \backslash U}
$$

ou seja, $\widetilde{L}^{+}(x) \cap A=\emptyset$. Assim obtemos uma contradição. Portanto $x \in \widetilde{P}_{w}^{+}(A)$.

Definição 3.3.4 Um subconjunto $A \subset X$ é chamado de $\widetilde{\pi}$-atrator fraco, se $\widetilde{P}_{w}^{+}(A)$ é uma vizinhança de $A$. E, $A \subset X$ é chamado de $\widetilde{\pi}$-atrator, se $\widetilde{P}^{+}(A)$ é uma vizinhança de $A$.

Proposição 3.3.5 Sejam $(X, \pi ; M, I)$ um sistema semidinâmico impulsivo e $A$ um subconjunto de $X$. Se $A$ é $\tilde{\pi}$-atrator então $\widetilde{P}^{+}(A)=\widetilde{P}_{w}^{+}(A)$.

Demonstração: Pela Proposição 3.3.2 temos que $\widetilde{P}^{+}(A) \subset \widetilde{P}_{w}^{+}(A)$. Provemos a outra inclusão. Seja $x \in \widetilde{P}_{w}^{+}(A)$. Como $\widetilde{P}^{+}(A)$ é uma vizinhança de $A$, existe $\tau \in \mathbb{R}_{+}$tal que $\tilde{\pi}(x, \tau) \in \widetilde{P}^{+}(A)$. Logo, dada uma vizinhança qualquer $U$ de $A$, existe $\lambda \in \mathbb{R}_{+}$tal que

$$
\tilde{\pi}(x,[\tau+\lambda,+\infty))=\tilde{\pi}(\tilde{\pi}(x, \tau),[\lambda,+\infty)) \subset U
$$

Portanto $x \in \widetilde{P}^{+}(A)$ e $\widetilde{P}_{w}^{+}(A) \subset \widetilde{P}^{+}(A)$.

No próximo teorema, estabelecemos condições para que as regiões de atração e atração fraca sejam conjuntos abertos.

Teorema 3.3.6 Sejam $(X, \pi ; M, I)$ um sistema semidinâmico impulsivo e $A$ um subconjunto de $X$.

i) Se $A$ é $\tilde{\pi}$-atrator fraco, então $\widetilde{P}_{w}^{+}(A) \backslash M$ é aberto;

ii) Se $A$ é $\widetilde{\pi}$-atrator, então $\widetilde{P}^{+}(A) \backslash M$ é aberto.

Demonstração: Pela Proposição 3.3.5 é suficiente provarmos o item $i$ ). Suponhamos que $A$ seja $\widetilde{\pi}$-atrator fraco. Então existe uma vizinhança aberta $U$ de $A$ tal que $U \subset \widetilde{P}_{w}^{+}(A)$. Agora, seja 
$x \in \widetilde{P}_{w}^{+}(A) \backslash M$. Então existe $t_{1}>0$ tal que $\tilde{\pi}\left(x, t_{1}\right) \in U$. Como $U$ é aberto podemos escolher $\lambda>0$ tal que $\tilde{\pi}\left(\tilde{\pi}\left(x, t_{1}\right), \lambda\right)=\pi\left(\tilde{\pi}\left(x, t_{1}\right), \lambda\right) \in U$ com $t_{1}+\lambda \neq \phi\left(x_{j}^{+}\right)$para todo $j \in\{0,1,2, \ldots\}$. Seja $V$ um aberto tal que $\tilde{\pi}\left(\tilde{\pi}\left(x, t_{1}\right), \lambda\right) \in V \subset U$. Como $\pi$ e $I$ são contínuas e $x \notin M$, existe um aberto $\mathscr{O}$ contendo $x$ tal que $\tilde{\pi}\left(\mathscr{O}, t_{1}+\lambda\right) \subset V$.

Afirmação: $\mathscr{O} \subset \widetilde{P}_{w}^{+}(A)$.

Com efeito, sejam $y \in \mathscr{O}$ e $W$ uma vizinhança qualquer de $A$. Então,

$$
\tilde{\pi}\left(y, t_{1}+\lambda\right) \in V \subset U \subset \widetilde{P}_{w}^{+}(A) .
$$

Logo, existe uma sequência $\left\{t_{n}\right\}_{n \geq 1}$ tal que $t_{n} \stackrel{n \rightarrow+\infty}{\longrightarrow}+\infty$ e $\tilde{\pi}\left(\tilde{\pi}\left(y, t_{1}+\lambda\right), t_{n}\right) \in W$ para todo $n \in \mathbb{N}^{*}$, ou seja,

$$
\tilde{\pi}\left(y, t_{1}+\lambda+t_{n}\right) \in W
$$

para todo $n \in \mathbb{N}^{*}$. Isto conclui que $y \in \widetilde{P}_{w}^{+}(A)$.

Portanto $x \in \mathscr{O} \subset \widetilde{P}_{w}^{+}(A)$ e o resultado está provado.

Definição 3.3.7 Sejam $(X, \pi ; M, I)$ um sistema semidinâmico impulsivo e $A$ um subconjunto de $X$. Dizemos que $A$ é assintoticamente $\tilde{\pi}$-estável, se $A$ é orbitalmente $\tilde{\pi}$-estável e $\tilde{\pi}$-atrator fraco.

Teorema 3.3.8 Sejam $(X, \pi ; M, I)$ um sistema semidinâmico impulsivo e $A$ um subconjunto de $X$. Se $A$ é assintoticamente $\widetilde{\pi}$-estável então $A$ é $\widetilde{\pi}$-atrator.

Demonstração: Basta mostrar que $\widetilde{P}^{+}(A)=\widetilde{P}_{w}^{+}(A)$. Como $\widetilde{P}^{+}(A) \subset \widetilde{P}_{w}^{+}(A)$, mostremos a outra inclusão. Sejam $x \in \widetilde{P}_{w}^{+}(A)$ e $U$ uma vizinhança qualquer de $A$. Como $A$ é orbitalmente $\tilde{\pi}$-estável, existe uma vizinhança positivamente $\tilde{\pi}$-invariante $V$ de $A$, tal que $V \subset U$ e $V \subset \widetilde{P}_{w}^{+}(A)$. Como $x \in \widetilde{P}_{w}^{+}(A)$, existe $\tau \in \mathbb{R}_{+}$tal que $\tilde{\pi}(x, \tau) \in V$. Logo

$$
\tilde{\pi}(x,[\tau,+\infty))=\tilde{\pi}(\tilde{\pi}(x, \tau),[0,+\infty)) \subset V \subset U
$$

e portanto $x \in \widetilde{P}^{+}(A)$. Assim $\widetilde{P}^{+}(A)=\widetilde{P}_{w}^{+}(A)$ é uma vizinhança de $A$ e $A$ é $\widetilde{\pi}$-atrator.

O corolário abaixo segue da Proposição 3.3.5 e do Teorema 3.3.8. 
Corolário 3.3.9 Seja $(X, \pi ; M, I)$ um sistema semidinâmico impulsivo e $A$ um subconjunto de $X$. Então $A$ é assintoticamente $\widetilde{\pi}$-estável se, e somente se, $A$ é $\widetilde{\pi}$-atrator e orbitalmente $\tilde{\pi}$-estável. 
$\underline{64} \quad$ Estabilidade 
Capítulo

\section{Estabilidade de Lyapunov}

Neste capítulo, estudamos a estabilidade de conjuntos fechados via funções do tipo de Lyapunov. Estas funções fornecem informações do comportamento assintótico de um sistema semidinâmico impulsivo mesmo sem conhecermos a solução do sistema. Apresentamos condições necessárias e suficientes para que um conjunto fechado seja estável em algum sentido. Os resultados deste capítulo são encontrados em [10].

Nesta seção vamos considerar as seguintes hipóteses adicionais para o sistema semidinâmico impulsivo $(X, \pi ; M, I)$.

H1) Para todo $x \in X$ e todo natural $k \geq 1, x_{k}^{+}$está definido e $M^{+}\left(x_{k}^{+}\right) \neq \emptyset$, isto é, a trajetória de $x$ tem infinitos pontos de descontinuidade e portanto para todo $x \in X$ temos $\phi(x)<+\infty$;

H2) $M \cap I(M)=\emptyset$.

\subsection{Critérios de estabilidade}

Proposição 4.1.1 Sejam $(X, \pi ; M, I)$ um sistema semidinâmico impulsivo e $\psi: X \rightarrow \mathbb{R}_{+}$uma função que satisfaz as seguintes condições:

i) Se $x \in X$ então $\psi(\pi(x, t)) \leq \psi(x)$ para todo $t \in[0, \phi(x)]$;

ii) Se $x \in M$ então $\psi(I(x)) \leq \psi(x)$. 
Então, dado $x \in X$, temos que $\psi(\widetilde{\pi}(x, t)) \leq \psi(x)$ para todo $t \geq 0$.

Demonstração: Seja $x \in X$. Pela condição $i$ ), temos

$$
\psi(\pi(x, t)) \leq \psi(x) \text { para todo } 0 \leq t \leq \phi(x) .
$$

Usando a condição $i i)$ para $t=\phi(x)$, obtemos a seguinte desigualdade

$$
\psi(\widetilde{\pi}(x, \phi(x)))=\psi(I(\pi(x, \phi(x)))) \leq \psi(\pi(x, \phi(x))) \leq \psi(x) .
$$

Portanto $\psi(\tilde{\pi}(x, t)) \leq \psi(x)$ para $0 \leq t \leq \phi(x)$.

Agora, seja $x_{1}^{+}=I\left(x_{1}\right)$, onde $x_{1}=\pi(x, \phi(x))$. Como

$$
\psi\left(\tilde{\pi}\left(x_{1}^{+}, t\right)\right) \leq \psi(x) \text { para todo } 0 \leq t \leq \phi\left(x_{1}^{+}\right)
$$

podemos repetir o processo feito acima, e portanto, obter a desigualdade

$$
\psi(\tilde{\pi}(x, t)) \leq \psi(x) \text { para todo } 0 \leq t \leq \phi(x)+\phi\left(x_{1}^{+}\right)
$$

De modo indutivo, segue que

$$
\psi(\tilde{\pi}(x, t)) \leq \psi(x) \text { para todo } t \geq 0
$$

Nas condições da Proposição 4.1.1, temos o seguinte resultado.

Corolário 4.1.2 Sejam $(X, \pi ; M, I)$ um sistema semidinâmico impulsivo e $\psi: X \rightarrow \mathbb{R}_{+}$uma função satisfazendo as condições $i)$ e $i i)$ da Proposição 4.1.1. Então $\psi(\widetilde{\pi}(x, s)) \leq \psi(\widetilde{\pi}(x, r))$ para todo $x \in X$ e para todos $s, r \in \mathbb{R}_{+}$tais que $s \geq r$.

Demonstração: Sejam $r, s \in \mathbb{R}_{+} \operatorname{com} s \geq r$ e $x \in X$. Pela Proposição 4.1 .1 temos

$$
\psi(\tilde{\pi}(\tilde{\pi}(x, r), t)) \leq \psi(\tilde{\pi}(x, r))
$$


para todo $t \geq 0$. Tomando $t=s-r$, obtemos $\psi(\tilde{\pi}(x, s)) \leq \psi(\tilde{\pi}(x, r))$.

O próximo resultado apresenta condições necessárias e suficientes para que um conjunto fechado seja $\tilde{\pi}$-estável.

Teorema 4.1.3 Sejam $(X, \pi ; M, I)$ um sistema semidinâmico impulsivo e $A$ um subconjunto fechado de $X$.

1. Se existe uma função $\psi: X \rightarrow \mathbb{R}_{+}$satisfazendo as seguintes propriedades:

a) $\psi$ é contínua em $X \backslash(M \backslash A)$;

b) Para todo $\varepsilon>0$ existe $\delta>0$ tal que se $\rho(x, A) \geq \varepsilon$ e $x \notin M$ então $\psi(x) \geq \delta$;

c) Para toda sequência $\left\{w_{n}\right\}_{n \geq 1} \subset X$ tal que $w_{n} \stackrel{n \rightarrow+\infty}{\longrightarrow} x \in A$ implica em $\psi\left(w_{n}\right) \stackrel{n \rightarrow+\infty}{\longrightarrow} 0$;

d) $\psi(\pi(x, t)) \leq \psi(x)$ se $x \in X \backslash M$ e $0 \leq t \leq \phi(x)$, e $\psi(I(x)) \leq \psi(x)$ para todo $x \in M$.

Então $A$ é $\widetilde{\pi}$-estável.

2. Reciprocamente, se $A$ é $\tilde{\pi}$-estável então existe uma função $\psi: X \rightarrow \mathbb{R}_{+}$satisfazendo as condições $a), b), c$ ) e $d$ ) acima.

Demonstração: Provemos o item 1. Dados $x \in A$ e $\varepsilon>0$, definamos

$$
\mu:=\inf \left\{\psi(w) \in \mathbb{R}_{+}: w \notin M \text { e } \rho(A, w) \geq \frac{\varepsilon}{2}\right\}
$$

Pelo item $b$ ), temos que $\mu>0$. Vamos considerar dois casos: quando $x \in \operatorname{int}(A)$ e quando $x \in \partial A$.

Primeiro consideremos o caso em que $x \in \operatorname{int}(A)$. Pela continuidade de $\psi$ em $A$ e pela condição c) segue que $\psi(x)=0$. Para $\mu>0$ existe $\delta_{1}>0$ tal que

$$
\psi(y)<\mu \text { sempre que } y \in B\left(x, \delta_{1}\right) \subset A \text {. }
$$

Mostraremos que $\tilde{\pi}\left(B\left(x, \delta_{1}\right),[0,+\infty)\right) \subset B(A, \varepsilon)$. Suponhamos o contrário, isto é, que existem $z \in B\left(x, \delta_{1}\right)$ e $t_{1} \in(0,+\infty)$ tais que

$$
\tilde{\pi}\left(z, t_{1}\right) \notin B(A, \varepsilon) .
$$


Notemos que $\tilde{\pi}\left(z, t_{1}\right) \notin M$ já que estamos considerando $M \cap I(M)=\emptyset$. Como $\rho\left(\tilde{\pi}\left(z, t_{1}\right), A\right) \geq \varepsilon$ segue da definição de $\mu$ que

$$
\psi\left(\tilde{\pi}\left(z, t_{1}\right)\right) \geq \mu
$$

Se $z \notin M$, então segue da Proposição 4.1 .1 e do fato de $z \in B\left(x, \delta_{1}\right)$ que

$$
\psi(\tilde{\pi}(z, t)) \leq \psi(z)<\mu \text { para todo } t \geq 0
$$

contradizendo (4.3).

Se $z \in M$ então existe $0<t_{2}<t_{1}$ tal que

$$
\tilde{\pi}\left(z, t_{2}\right)=\pi\left(z, t_{2}\right) \in B\left(x, \delta_{1}\right) \backslash M
$$

Por (4.1) e pelo Corolário 4.1.2 obtemos que $\psi\left(\widetilde{\pi}\left(z, t_{1}\right)\right) \leq \psi\left(\widetilde{\pi}\left(z, t_{2}\right)\right)<\mu$ o que contradiz (4.3). Portanto $\tilde{\pi}\left(B\left(x, \delta_{1}\right),[0,+\infty)\right) \subset B(A, \varepsilon)$.

Agora, analisemos o caso em que $x \in \partial A$. Como $\psi$ é contínua em $X \backslash(M \backslash A)$ e temos a condição $c$ ), existe um $\delta_{2}>0, \delta_{2}<\varepsilon$, tal que $\psi(y)<\mu$ para todo $y \in B\left(x, \delta_{2}\right) \backslash M$. Suponhamos que $\tilde{\pi}\left(B\left(x, \delta_{2}\right),[0,+\infty)\right) \nsubseteq B(A, \varepsilon)$, então existem $z \in B\left(x, \delta_{2}\right)$ e $t_{2} \in(0,+\infty)$ tais que $\tilde{\pi}\left(z, t_{2}\right) \notin B(A, \varepsilon)$. Logo $\rho\left(\tilde{\pi}\left(z, t_{2}\right), A\right) \geq \varepsilon, \tilde{\pi}\left(z, t_{2}\right) \notin M$ pois $M \cap I(M)=\emptyset$ e portanto

$$
\psi\left(\widetilde{\pi}\left(z, t_{2}\right)\right) \geq \inf \left\{\psi(w): w \notin M \text { e } \rho(w, A) \geq \frac{\varepsilon}{2}\right\}=\mu
$$

Se $z \in B\left(x, \delta_{2}\right) \backslash M$, então $\psi(\tilde{\pi}(z, t)) \leq \psi(z)<\mu$ para todo $t \geq 0$. Portanto $\psi\left(\tilde{\pi}\left(z, t_{2}\right)\right)<\mu$ o que contradiz (4.4). Notemos, também, que se $z \in B\left(x, \delta_{2}\right) \cap M$, então existe $\tau>0, \tau<t_{2}$, tal que $\tilde{\pi}(z,(0, \tau))=\pi(z,(0, \tau)) \subset B\left(x, \delta_{2}\right) \backslash M$. Tomando $t^{*}, 0<t^{*}<\tau$, segue que

$$
\psi\left(\tilde{\pi}\left(\pi\left(z, t^{*}\right), t\right)\right)<\psi\left(\pi\left(z, t^{*}\right)\right)<\mu
$$

para todo $t \geq 0$. Assim $\psi\left(\widetilde{\pi}\left(z, t_{2}\right)\right)=\psi\left(\widetilde{\pi}\left(\pi\left(z, t^{*}\right), t_{2}-t^{*}\right)\right)<\mu$ e isto contradiz (4.4). Portanto $\tilde{\pi}\left(B\left(x, \delta_{2}\right),[0,+\infty)\right) \subset B(A, \varepsilon)$. 
Desta forma concluímos que $A$ é $\widetilde{\pi}$-estável.

2. Definamos $\psi: X \rightarrow \mathbb{R}_{+}$por:

$$
\psi(x)= \begin{cases}\sup _{k \geq 0}\left(\sup _{0 \leq t \leq \phi\left(x_{k}^{+}\right)} \frac{\rho\left(\pi\left(x_{k}^{+}, t\right), A\right)}{1+\rho\left(\pi\left(x_{k}^{+}, t\right), A\right)}\right) & \text { se } x \in X \backslash M \\ \sup _{k \geq 0}\left(\sup _{0 \leq t \leq \phi\left((I(x))_{k}^{+}\right)} \frac{\rho\left(\pi\left((I(x))_{k}^{+}, t\right), A\right)}{1+\rho\left(\pi\left((I(x))_{k}^{+}, t\right), A\right)}\right) & \text { se } x \in M .\end{cases}
$$

Notemos que se $x \in M$ então função $\psi$ é definida por $\psi(x)=\psi(I(x))$.

Mostremos que a função $\psi$ satisfaz as condições $a), b), c$ ) e $d$ ) do enunciado.

a) Sejam $x \in X \backslash M$ e $\left\{w_{n}\right\}_{n \geq 1} \subset X$ uma sequência tal que $w_{n} \stackrel{n \rightarrow+\infty}{\longrightarrow} x$. Podemos supor que $w_{n} \notin M$ para todo $n \in \mathbb{N}$, pois $M$ é fechado. Mostraremos que

$$
\psi\left(w_{n}\right) \stackrel{n \rightarrow+\infty}{\longrightarrow} \psi(x)
$$

Como $w_{n} \stackrel{n \rightarrow+\infty}{\longrightarrow} x$, segue que $\phi\left(w_{n}\right) \stackrel{n \rightarrow+\infty}{\longrightarrow} \phi(x) \mathrm{e}$

$$
\sup _{0 \leq t \leq \phi\left(w_{n}\right)} \frac{\rho\left(\pi\left(w_{n}, t\right), A\right)}{1+\rho\left(\pi\left(w_{n}, t\right), A\right)} \stackrel{n \rightarrow+\infty}{\longrightarrow} \sup _{0 \leq t \leq \phi(x)} \frac{\rho(\pi(x, t), A)}{1+\rho(\pi(x, t), A)} .
$$

Pela continuidade das funções $I$ e $\phi$, e pelo fato de que $I(M) \cap M=\emptyset$, obtemos

$$
\left(w_{n}\right)_{1}^{+}=I\left(\pi\left(w_{n}, \phi\left(w_{n}\right)\right)\right) \stackrel{n \rightarrow+\infty}{\longrightarrow} I(\pi(x, \phi(x)))=x_{1}^{+} \notin M .
$$

$\operatorname{Logo} \phi\left(\left(w_{n}\right)_{1}^{+}\right) \stackrel{n \rightarrow+\infty}{\longrightarrow} \phi\left(x_{1}^{+}\right) \mathrm{e}$

$$
\sup _{0 \leq t \leq \phi\left(\left(w_{n}\right)_{1}^{+}\right)} \frac{\rho\left(\pi\left(\left(w_{n}\right)_{1}^{+}, t\right), A\right)}{1+\rho\left(\pi\left(\left(w_{n}\right)_{1}^{+}, t\right), A\right)} \stackrel{n \rightarrow+\infty}{\longrightarrow} \sup _{0 \leq t \leq \phi\left(x_{1}^{+}\right)} \frac{\rho\left(\pi\left(x_{1}^{+}, t\right), A\right)}{1+\rho\left(\pi\left(x_{1}^{+}, t\right), A\right)}
$$

Repetindo o processo feito acima, temos que para cada $k \geq 0$, vale a convergência

$$
\sup _{0 \leq t \leq \phi\left(\left(w_{n}\right)_{k}^{+}\right)} \frac{\rho\left(\pi\left(\left(w_{n}\right)_{k}^{+}, t\right), A\right)}{1+\rho\left(\pi\left(\left(w_{n}\right)_{k}^{+}, t\right), A\right)} \stackrel{n \rightarrow+\infty}{\longrightarrow} \sup _{0 \leq t \leq \phi\left(x_{k}^{+}\right)} \frac{\rho\left(\pi\left(x_{k}^{+}, t\right), A\right)}{1+\rho\left(\pi\left(x_{k}^{+}, t\right), A\right)}
$$




\section{Portanto}

$$
\sup _{k \geq 0}\left(\sup _{0 \leq t \leq \phi\left(\left(w_{n}\right)_{k}^{+}\right)} \frac{\rho\left(\pi\left(\left(w_{n}\right)_{k}^{+}, t\right), A\right)}{1+\rho\left(\pi\left(\left(w_{n}\right)_{k}^{+}, t\right), A\right)}\right) \stackrel{n \rightarrow+\infty}{\longrightarrow} \sup _{k \geq 0}\left(\sup _{0 \leq t \leq \phi\left(x_{k}^{+}\right)} \frac{\rho\left(\pi\left(x_{k}^{+}, t\right), A\right)}{1+\rho\left(\pi\left(x_{k}^{+}, t\right), A\right)}\right),
$$

isto é,

$$
\psi\left(w_{n}\right) \stackrel{n \rightarrow+\infty}{\longrightarrow} \psi(x) .
$$

Assim $\psi$ é contínua em $X \backslash M$.

Agora, mostremos que $\psi$ é contínua em $A \cap M$. Dado $x \in A \cap M$, segue da Observação 3.1.4 que o subconjunto $A$ é positivamente $\tilde{\pi}$-invariante, ou seja, $\widetilde{\pi}^{+}(x) \subset A$. Logo, para todo $k \geq 0$ e $t \in\left[0, \phi\left(x_{k}^{+}\right)\right]$, temos $\pi\left(x_{k}^{+}, t\right) \in A$. Então $\rho\left(\pi\left(x_{k}^{+}, t\right), A\right)=0$ para todo $k \geq 0$ e para todo $t \in\left[0, \phi\left(x_{k}^{+}\right)\right]$. Portanto $\psi(x)=0$ para todo $x \in A \cap M$.

Seja $\left\{z_{n}\right\}_{n \geq 1}$ uma sequência em $X$ tal que $z_{n} \stackrel{n \rightarrow+\infty}{\longrightarrow} x$. Pela $\tilde{\pi}$-estabilidade de $A$, dado $\varepsilon>0$ existe $\delta(x, \varepsilon)>0$ tal que $\tilde{\pi}(B(A, \delta),[0,+\infty)) \subset B(A, \varepsilon)$. Notemos que existe um natural $n_{0}$ tal que $z_{n} \in$ $B(A, \delta)$ para todo $n \geq n_{0}$. Então $\tilde{\pi}^{+}\left(z_{n}\right) \subset B(A, \varepsilon)$ para todo $n \geq n_{0}$. Segue que $\rho\left(\pi\left(\left(z_{n}\right)_{k}^{+}, t\right), A\right)<\varepsilon$ para todo $t \in\left[0, \phi\left(\left(z_{n}\right)_{k}^{+}\right)\right], k=0,1,2, \ldots$, e para todo $n \geq n_{0}$. Portanto $\psi\left(z_{n}\right)<\varepsilon$ para todo $n \geq n_{0}$. Como $\varepsilon$ é arbitrário temos

$$
\psi\left(z_{n}\right) \stackrel{n \rightarrow+\infty}{\longrightarrow} 0=\psi(x)
$$

e assim segue a continuidade de $\psi$ em $X \backslash(M \backslash A)$.

b) Dado $\varepsilon>0$ tomemos $\delta=\frac{\varepsilon}{1+\varepsilon}$. Se $x \in X \backslash M$ e $\rho(x, A) \geq \varepsilon$, obtemos

$$
\frac{\rho\left(\pi\left(x_{0}^{+}, 0\right), A\right)}{1+\rho\left(\pi\left(x_{0}^{+}, 0\right), A\right)} \geq \delta
$$

onde $\pi\left(x_{0}^{+}, 0\right)=x$. Assim

$$
\psi(x)=\sup _{k \geq 0}\left(\sup _{0 \leq t \leq \phi\left(x_{k}^{+}\right)} \frac{\rho\left(\pi\left(x_{k}^{+}, t\right), A\right)}{1+\rho\left(\pi\left(x_{k}^{+}, t\right), A\right)}\right) \geq \delta .
$$

c) Provamos do item $a)$ que $\psi$ é contínua em $X \backslash(M \backslash A)$. Logo $\psi$ é contínua em $A$. Provamos, também, no item $a$ ) que $\psi(x)=0$ se $x \in A$, pois $A$ é $\widetilde{\pi}$-estável. Portanto se $\left\{w_{n}\right\}_{n \geq 1}$ é uma sequência 
de $X$ tal que $w_{n} \stackrel{n \rightarrow+\infty}{\longrightarrow} x$ então $\psi\left(w_{n}\right) \stackrel{n \rightarrow+\infty}{\longrightarrow} \psi(x)=0$.

d) Sejam $x \in X \backslash M$ e $s \in[0, \phi(x))$. Definamos $y=\pi(x, s)$. Então, para cada $k \geq 1$, temos

$$
y_{k}^{+}=x_{k}^{+}
$$

Consequentemente $\phi\left(y_{k}^{+}\right)=\phi\left(x_{k}^{+}\right)$para todo $k \geq 1$, e assim

$$
\sup _{0 \leq t \leq \phi\left(x_{k}^{+}\right)} \frac{\rho\left(\pi\left(x_{k}^{+}, t\right), A\right)}{1+\rho\left(\pi\left(x_{k}^{+}, t\right), A\right)}=\sup _{0 \leq t \leq \phi\left(y_{k}^{+}\right)} \frac{\rho\left(\pi\left(y_{k}^{+}, t\right), A\right)}{1+\rho\left(\pi\left(y_{k}^{+}, t\right), A\right)}
$$

para todo $k \geq 1$. Como $\phi\left(y_{0}^{+}\right) \leq \phi\left(x_{0}^{+}\right)$segue que

$$
\sup _{0 \leq t \leq \phi\left(x_{0}^{+}\right)} \frac{\rho\left(\tilde{\pi}\left(x_{0}^{+}, t\right), A\right)}{1+\rho\left(\tilde{\pi}\left(x_{0}^{+}, t\right), A\right)} \geq \sup _{0 \leq t \leq \phi\left(y_{0}^{+}\right)} \frac{\rho\left(\tilde{\pi}\left(y_{0}^{+}, t\right), A\right)}{1+\rho\left(\tilde{\pi}\left(y_{0}^{+}, t\right), A\right)}
$$

Portanto,

$$
\sup _{k \geq 0}\left(\sup _{0 \leq t \leq \phi\left(y_{k}^{+}\right)} \frac{\rho\left(\tilde{\pi}\left(y_{k}^{+}, t\right), A\right)}{1+\rho\left(\widetilde{\pi}\left(y_{k}^{+}, t\right), A\right)}\right) \leq \sup _{k \geq 0}\left(\sup _{0 \leq t \leq \phi\left(x_{k}^{+}\right)} \frac{\rho\left(\tilde{\pi}\left(x_{k}^{+}, t\right), A\right)}{1+\rho\left(\tilde{\pi}\left(x_{k}^{+}, t\right), A\right)}\right),
$$

ou seja,

$$
\psi(\pi(x, s))=\psi(y) \leq \psi(x)
$$

para $0 \leq s<\phi(x)$. Se $s=\phi(x)$, temos que $\pi(x, \phi(x)) \in M$. Logo

$$
\begin{aligned}
\psi(\pi(x, \phi(x))) & =\psi\left(I\left(x_{1}\right)\right)=\psi\left(x_{1}^{+}\right)=\sup _{k \geq 1}\left(\sup _{0 \leq t \leq \phi\left(x_{k}^{+}\right)} \frac{\rho\left(\pi\left(x_{k}^{+}, t\right), A\right)}{1+\rho\left(\pi\left(x_{k}^{+}, t\right), A\right)}\right) \leq \\
& \leq \sup _{k \geq 0}\left(\sup _{0 \leq t \leq \phi\left(x_{k}^{+}\right)} \frac{\rho\left(\pi\left(x_{k}^{+}, t\right), A\right)}{1+\rho\left(\pi\left(x_{k}^{+}, t\right), A\right)}\right)=\psi(x) .
\end{aligned}
$$

Portanto $\psi(\pi(x, t)) \leq \psi(x)$ para todo $x \in X \backslash M$ e para todo $0 \leq t \leq \phi(x)$. Por outro lado, se $x \in M$, segue da definição de $\psi$ que $\psi(x)=\psi(I(x))$. E o teorema está provado.

Suponhamos, no teorema acima, que o conjunto impulsivo $M$ está contido no conjunto $A$, então segue que $\psi$ é contínua em $X$. Veja o próximo resultado. 
Corolário 4.1.4 Sejam $(X, \pi ; M, I)$ um sistema semidinâmico impulsivo e $A$ um subconjunto fechado de $X$ tal que $M \subset A$. Então $A$ é $\widetilde{\pi}$-estável se, e somente se, existe uma função $\psi: X \rightarrow \mathbb{R}_{+}$ que satisfaz as seguintes propriedades:

a) $\psi$ é contínua em $X$;

b) Para todo $\varepsilon>0$ existe um $\delta>0$ tal que $\psi(x) \geq \delta$ sempre que $\rho(x, A) \geq \varepsilon$ e $x \notin M$;

c) Para qualquer sequência $\left\{w_{n}\right\}_{n \geq 1} \subset X$ tal que $w_{n} \stackrel{n \rightarrow+\infty}{\longrightarrow} x \in A$ implica $\psi\left(w_{n}\right) \stackrel{n \rightarrow+\infty}{\longrightarrow} 0$;

d) $\psi(\pi(x, t)) \leq \psi(x)$ se $x \in X \backslash M$ e $t \geq 0$, e $\psi(I(x)) \leq \psi(x)$ para todo $x \in M$.

O teorema seguinte apresenta condições necessárias e suficientes para equi $\tilde{\pi}$-estabilidade de subconjuntos fechados de $X$.

Teorema 4.1.5 Sejam $(X, \pi ; M, I)$ um sistema semidinâmico impulsivo e $A$ um subconjunto fechado de $X$. Suponhamos que $I(M \backslash A) \subset(X \backslash A) \backslash M$.

1. Se existe uma função $\psi: X \rightarrow \mathbb{R}_{+}$que satisfaz as seguintes propriedades:

a) $\psi$ é semicontínua inferiormente em $X \backslash M$;

b) $\psi(x)=0$ para $x \in A$ e $\psi(x)>0$ para todo $x \notin A \cup M$;

c) Para todo $\varepsilon>0$ existe um $\delta>0$ tal que $\psi(x) \leq \varepsilon$ sempre que $\rho(x, A) \leq \delta$;

d) $\psi(\pi(x, t)) \leq \psi(x)$ se $x \in X \backslash M$ e $0 \leq t \leq \phi(x)$, e $\psi(I(x)) \leq \psi(x)$ para todo $x \in M$.

Então $A$ é equi $\widetilde{\pi}$-estável.

2. Reciprocamente, se $A$ é equi $\widetilde{\pi}$-estável então existe uma função $\psi: X \rightarrow \mathbb{R}_{+}$satisfazendo as condições $a), b), c)$ e $d$ ) acima.

Demonstração: 1 . Suponhamos que $x \notin A$. Temos dois casos a considerar: quando $x \in M$ e quando $x \notin M$.

Suponhamos, primeiramente, que $x \notin M$. Seja $\rho(x, A)=\varepsilon>0$. Como $x \notin M$, segue pela condição $b$ ) que $\psi(x)>0$. Seja $\psi(x)=\mu$. A condição $c$ ) garante a existência de um número $\eta>0$ 
tal que

$$
\psi(y) \leq \frac{\mu}{2} \quad \text { sempre que } \quad \rho(y, A) \leq \eta
$$

Seja $\delta>0, \delta<\min \{\eta, \varepsilon\}$. Mostremos que $x \notin \overline{\widetilde{\pi}(B(A, \delta),[0,+\infty))}$. De fato, suponhamos o contrário. Então existem sequências $\left\{y_{n}\right\}_{n \geq 1} \subseteq B(A, \delta)$ e $\left\{T_{n}\right\}_{n \geq 1} \subseteq[0,+\infty)$ tais que

$$
\tilde{\pi}\left(y_{n}, T_{n}\right) \stackrel{n \rightarrow+\infty}{\longrightarrow} x
$$

Como $x \notin M,\{x\}$ é compacto e $M$ é fechado, existe $\rho>0$ tal que $B(x, \rho) \cap M=\emptyset$. Além disso, existe um inteiro $n_{0}>0$ tal que $\tilde{\pi}\left(y_{n}, T_{n}\right) \in B(x, \rho)$ para todo $n>n_{0}$. Segue da semicontinuidade inferior de $\psi$ em $X \backslash M$ que existe um inteiro $n_{1}>n_{0}$ tal que

$$
\psi\left(\tilde{\pi}\left(y_{n}, T_{n}\right)\right)>\psi(x)-\frac{\mu}{3}
$$

para todo $n \geq n_{1}$. Como $\psi(x)=\mu$, temos

$$
\psi\left(\widetilde{\pi}\left(y_{n_{1}}, T_{n_{1}}\right)\right)>\frac{2 \mu}{3}
$$

Pela Proposição 4.1.1 segue que $\psi(\tilde{\pi}(w, t)) \leq \psi(w)$ para todo $t \geq 0$ e para todo $w \in X \backslash M$. Usando este fato e (4.6) obtemos

$$
\psi\left(\widetilde{\pi}\left(y_{n_{1}}, T_{n_{1}}\right)\right) \leq \psi\left(y_{n_{1}}\right) \leq \frac{\mu}{2}
$$

e isto contradiz (4.7). Portanto $x \notin \overline{\widetilde{\pi}(B(A, \delta),[0,+\infty))}$.

Agora, consideremos o caso em que $x \in M$. Suponhamos que $x \in \overline{\widetilde{\pi}(B(A, \delta),[0,+\infty))}$ para todo $\delta>0$. Então, existem sequências $\left\{w_{n}^{\delta}\right\}_{n \geq 1} \subset B(A, \delta)$ e $\left\{t_{n}^{\delta}\right\}_{n \geq 1} \subset[0,+\infty)$ tais que

$$
\tilde{\pi}\left(w_{n}^{\delta}, t_{n}^{\delta}\right) \stackrel{n \rightarrow+\infty}{\rightarrow} x
$$

para todo $\delta>0$. Como cada elemento de $M$ satisfaz a condição STC, podemos considerar dois casos: 
Caso 1: Existe uma subsequência de $\left\{\tilde{\pi}\left(w_{n}^{\delta}, t_{n}^{\delta}\right)\right\}_{n \geq 1}$, denotada por $\left\{\tilde{\pi}\left(w_{n_{k}}^{\delta}, t_{n_{k}}^{\delta}\right)\right\}_{k \geq 1}$, tal que

$$
\pi\left(\widetilde{\pi}\left(w_{n_{k}}^{\delta}, t_{n_{k}}^{\delta}\right), \phi\left(\tilde{\pi}\left(w_{n_{k}}^{\delta}, t_{n_{k}}^{\delta}\right)\right) \stackrel{k \rightarrow+\infty}{\rightarrow} x\right.
$$

Neste caso, como $\pi\left(\widetilde{\pi}\left(w_{n_{k}}^{\delta}, t_{n_{k}}^{\delta}\right), \phi\left(\widetilde{\pi}\left(w_{n_{k}}^{\delta}, t_{n_{k}}^{\delta}\right)\right)\right) \in M$ e $I$ é contínua, temos

$$
\widetilde{\pi}\left(\widetilde{\pi}\left(w_{n_{k}}^{\delta}, t_{n_{k}}^{\delta}\right), \phi\left(\widetilde{\pi}\left(w_{n_{k}}^{\delta}, t_{n_{k}}^{\delta}\right)\right)\right) \stackrel{k \rightarrow+\infty}{\rightarrow} I(x),
$$

isto é,

$$
\widetilde{\pi}\left(w_{n_{k}}^{\delta}, t_{n_{k}}^{\delta}+\phi\left(\widetilde{\pi}\left(w_{n_{k}}^{\delta}, t_{n_{k}}^{\delta}\right)\right)\right) \stackrel{k \rightarrow+\infty}{\rightarrow} I(x)
$$

Por outro lado, como $x \in M \backslash A$, segue que $I(x) \notin A \cup M$ pois por hipótese temos a condição $I(M \backslash A) \subset(X \backslash A) \backslash M$. Então pela primeira parte desta demonstração, existe um $\bar{\delta}>0$ tal que $I(x) \notin \overline{\widetilde{\pi}(B(A, \bar{\delta}),[0,+\infty))}$. Mas isso contradiz (4.8). Logo existe $\delta>0$ tal que

$$
x \notin \overline{\widetilde{\pi}(B(A, \delta),[0,+\infty))} .
$$

Caso 2: Existe uma subsequência de $\left\{\tilde{\pi}\left(w_{n}^{\delta}, t_{n}^{\delta}\right)\right\}_{n \geq 1}$, também denotada por $\left\{\tilde{\pi}\left(w_{n_{k}}^{\delta}, t_{n_{k}}^{\delta}\right)\right\}_{k \geq 1}$, tal que $\phi\left(\tilde{\pi}\left(w_{n_{k}}^{\delta}, t_{n_{k}}^{\delta}\right)\right) \stackrel{k \rightarrow+\infty}{\rightarrow} \phi(x)$. Neste caso,

$$
\pi\left(\widetilde{\pi}\left(w_{n_{k}}^{\delta}, t_{n_{k}}^{\delta}\right), t\right) \stackrel{k \rightarrow+\infty}{\longrightarrow} \pi(x, t)
$$

para todo $0 \leq t<\phi(x)$. Seja $0<t_{0}<\phi(x)$ tal que $\pi\left(x, t_{0}\right) \notin A \cup M$. Novamente pela primeira parte desta demonstração, existe $\tilde{\delta}>0$ tal que

$$
\pi\left(x, t_{0}\right) \notin \overline{\widetilde{\pi}(B(A, \tilde{\delta}),[0,+\infty))}
$$

Assim temos uma contradição pois

$$
\widetilde{\pi}\left(w_{n_{k}}^{\tilde{\delta}}, t_{n_{k}}^{\tilde{\delta}}+t_{0}\right)=\pi\left(\widetilde{\pi}\left(w_{n_{k}}^{\tilde{\delta}}, t_{n_{k}}^{\tilde{\delta}}\right), t_{0}\right) \stackrel{k \rightarrow+\infty}{\rightarrow} \pi\left(x, t_{0}\right)
$$

Portanto existe $\delta>0$ tal que $x \notin \overline{\widetilde{\pi}(B(A, \delta),[0,+\infty))}$. 
Concluímos, então, que $A$ é equi $\tilde{\pi}$-estável.

2. Consideremos a função $\psi: X \rightarrow \mathbb{R}_{+}$definida por

$$
\psi(x)=\left\{\begin{array}{llc}
\sup \left\{\delta>0: x \notin \overline{\tilde{\pi}^{+}(B(A, \delta))}\right\} & \text { se } & x \in X \backslash(A \cup M), \\
0 & \text { se } & x \in A \\
\sup \left\{\delta>0: I(x) \notin \overline{\tilde{\pi}^{+}(B(A, \delta))}\right\} & \text { se } & x \in(X \backslash A) \cap M
\end{array}\right.
$$

Mostremos que a função $\psi$ satisfaz as condições $a), b), c$ ) e $d$ ).

a) Seja $x \in X \backslash M$. Tomemos uma sequência $\left\{w_{n}\right\}_{n \geq 1} \subset X$ tal que $w_{n} \stackrel{n \rightarrow+\infty}{\longrightarrow} x$. Seja $\delta>0$ arbitrário tal que $x \notin \overline{\widetilde{\pi}^{+}(B(A, \delta))}$. Logo existe $\mu=\mu(x, \delta)>0$ tal que

$$
B(x, \mu) \cap \overline{\widetilde{\pi}^{+}(B(A, \delta))}=\emptyset .
$$

Assim, existe $n_{0} \in \mathbb{N}$ tal que $w_{n} \notin \overline{\widetilde{\pi}^{+}(B(A, \delta))}$ para todo $n>n_{0}$. Portanto

$$
\liminf _{n \rightarrow+\infty} \psi\left(w_{n}\right) \geq \psi(x)
$$

b) Pela definição de $\psi$ temos que $\psi(x)=0$ se $x \in A$. Agora, seja $x \notin A$. Pela equi $\widetilde{\pi}$-estabilidade de $A$, existe $\delta_{0}>0$ tal que $x \notin \overline{\tilde{\pi}\left(B\left(A, \delta_{0}\right),[0,+\infty)\right)}$. Se $x \notin M$ então $\psi(x) \geq \delta_{0}>0$. Agora, se $x \in M$, segue da hipótese $I(M \backslash A) \subset(X \backslash A) \backslash M$ que $I(x) \notin A \cup M$. Portanto existe $\delta_{1}>0$ tal que $I(x) \notin \overline{\widetilde{\pi}\left(B\left(A, \delta_{1}\right),[0,+\infty)\right)}$ e $\psi(x)=\psi(I(x)) \geq \delta_{1}>0$. Logo $\psi(x)>0$ sempre que $x \notin A \cup M$.

c) Notemos que $\psi(x) \leq \rho(x, A)$. Então, dado $\varepsilon>0$, tomando $\delta=\varepsilon$, segue que se $\rho(x, A) \leq \delta$ então

$$
\psi(x) \leq \rho(x, A) \leq \delta=\varepsilon
$$

d) Seja $x \in X \backslash M$. Se $x \in A$ então $\tilde{\pi}^{+}(x) \subset A$, pois $A$ é equi $\tilde{\pi}$-estável, e portanto

$$
\psi(\pi(t, x))=0=\psi(x) \text { para todo } 0 \leq t \leq \phi(x)
$$

Agora, se $x \notin A$, seja $\mu=\rho(x, A)>0$. Suponhamos que exista $t \in(0, \phi(x))$ tal que 
$\psi(\pi(x, t))>\psi(x)$, isto é,

$$
\psi(\pi(x, t))=\sup \left\{\delta>0: \pi(x, t) \notin \overline{\widetilde{\pi}^{+}(B(A, \delta))}\right\}>\psi(x)
$$

Logo existe $\delta_{0}>0$ tal que

$$
x \in \overline{\widetilde{\pi}^{+}\left(B\left(A, \delta_{0}\right)\right)} \text { e } \pi(x, t) \notin \overline{\widetilde{\pi}^{+}\left(B\left(A, \delta_{0}\right)\right)} .
$$

Daí existe sequências $\left\{w_{n}\right\}_{n \geq 1} \subset B\left(A, \delta_{0}\right)$ e $\left\{t_{n}\right\}_{n \geq 1} \subset \mathbb{R}_{+}$tais que

$$
\tilde{\pi}\left(x_{n}, t_{n}\right) \stackrel{n \rightarrow+\infty}{\longrightarrow} x .
$$

Pelo Lema 2.4 .5 , existe uma sequência $\left\{\varepsilon_{n}\right\}_{n \geq 1} \subset \mathbb{R}$ tal que $\varepsilon_{n} \stackrel{n \rightarrow+\infty}{\longrightarrow} 0 \mathrm{e}$

$$
\tilde{\pi}\left(x_{n}, t_{n}+\varepsilon_{n}+t\right) \stackrel{n \rightarrow+\infty}{\longrightarrow} \pi(x, t)
$$

ou seja, $\pi(x, t) \in \overline{\tilde{\pi}^{+}\left(B\left(A, \delta_{0}\right)\right)}$ e isto é um absurdo. Portanto $\psi(\pi(x, t)) \leq \psi(x)$ para todo $t \in[0, \phi(x))$.

Agora, seja $\left\{s_{n}\right\}_{n \geq 1} \subset[0, \phi(x))$ uma sequência crescente tal que $s_{n} \stackrel{n \rightarrow+\infty}{\longrightarrow} \phi(x)$. Notemos que

$$
\psi\left(\pi\left(x, s_{n}\right)\right) \leq \psi(x)
$$

para todo $n \in \mathbb{N}^{*}$. Como $\psi$ é semicontínua inferiormente, temos

$$
\psi(\pi(x, \phi(x))) \leq \liminf _{n \rightarrow+\infty} \psi\left(\pi\left(x, s_{n}\right)\right) \leq \psi(x)
$$

Portanto, $\psi(\pi(x, t)) \leq \psi(x)$ para todo $t \in[0, \phi(x)]$.

Por outro lado, se $x \in M$ segue que

$$
\psi(x)=\sup \left\{\delta>0: I(x) \notin \overline{\widetilde{\pi}^{+}(B(A, \delta))}\right\}=\psi(I(x))
$$


Corolário 4.1.6 Sejam $(X, \pi ; M, I)$ um sistema semidinâmico impulsivo e $A$ um subconjunto fechado de $X$ tal que $M \subset A$. Então $A$ é equi $\widetilde{\pi}$-estável se, e somente se, existe uma função $\psi: X \rightarrow \mathbb{R}_{+}$que satisfaz as seguintes propriedades:

a) $\psi$ é semicontínua inferiormente em $X$;

b) $\psi(x)=0$ para todo $x \in A$ e $\psi(x)>0$ para todo $x \notin A \cup M$;

c) Para todo $\varepsilon>0$ existe um $\delta>0$ tal que $\psi(x) \leq \varepsilon$ sempre que $\rho(x, A) \leq \delta$;

d) $\psi(\pi(x, t)) \leq \psi(x)$ se $x \in X \backslash M$ e $t \geq 0$, e $\psi(I(x)) \leq \psi(x)$ para todo $x \in M$.

A seguir, apresentamos um resultado auxiliar que será utilizado no próximo teorema.

Lema 4.1.7 Sejam $(X, \pi ; M, I)$ um sistema semidinâmico impulsivo e $A$ um subconjunto fechado de $X$. Seja $\psi: X \rightarrow \mathbb{R}_{+}$uma função contínua em $X \backslash(M \backslash A)$ que satisfaz as seguintes propriedades:

a) Para todo $\varepsilon>0$ existe um $\delta>0$ tal que $\psi(x) \geq \delta$ sempre que $\rho(x, A) \geq \varepsilon$ e $x \notin M$.

b) Para todo $\varepsilon>0$ existe um $\delta>0$ tal que $\psi(x) \leq \varepsilon$ sempre que $\rho(x, A) \leq \delta$.

Suponhamos que exista um $\widetilde{\delta}>0$ tal que $\psi(\widetilde{\pi}(w, t)) \leq \psi(w)$ para todo $t \geq 0$ e $w \in \overline{B(A, \widetilde{\delta}) \backslash M}$. Então existe um $\bar{\delta}>0,0<\bar{\delta}<\widetilde{\delta}$, tal que $\widetilde{\pi}(B(A, \bar{\delta}),[0,+\infty)) \subset B(A, \widetilde{\delta})$.

Demonstração: Suponhamos que não exista tal número $\bar{\delta}<\widetilde{\delta}$ tal que $\tilde{\pi}(B(A, \bar{\delta}),[0,+\infty)) \subset$ $B(A, \widetilde{\delta})$. Então dado qualquer sequência $\left\{\delta_{n}\right\}_{n \geq 1} \subset \mathbb{R}_{+}$tal que $\delta_{n} \stackrel{n \rightarrow+\infty}{\longrightarrow} 0$ obtemos

$$
\tilde{\pi}\left(B\left(A, \delta_{n}\right),[0,+\infty)\right) \nsubseteq B(A, \widetilde{\delta}),
$$

ou seja, para cada $n \in \mathbb{N}^{*}$ existem $w_{n} \in B\left(A, \delta_{n}\right)$ e $t_{n} \in(0,+\infty)$ tais que $\widetilde{\pi}\left(w_{n}, t_{n}\right) \notin B(A, \widetilde{\delta})$.

Agora, definamos $\mu:=\inf \{\psi(x): x \notin M$ e $\rho(x, A) \geq \widetilde{\delta}\}$. Segue do item $a)$ que $\mu>0$. Como $\tilde{\pi}\left(w_{n}, t_{n}\right) \notin M$ e $\rho\left(\widetilde{\pi}\left(w_{n}, t_{n}\right), A\right) \geq \widetilde{\delta}$ obtemos

$$
\psi\left(\tilde{\pi}\left(w_{n}, t_{n}\right)\right)>\mu
$$


para todo $n \in \mathbb{N}^{*}$. Seja $\mu>0$ obtido acima, segue do item $b$ ) que existe $v>0, v<\widetilde{\delta}$, tal que

$$
\psi(y) \leq \frac{\mu}{2} \quad \text { sempre que } \quad \rho(y, A) \leq v
$$

Tomemos $n_{0} \in \mathbb{N}$ tal que $\delta_{n}<v$ para todo $n \geq n_{0}$. Logo $B\left(A, \delta_{n_{0}}\right) \subset B(A, v)$ e por (4.10)

$$
\psi\left(w_{n_{0}}\right) \leq \frac{\mu}{2}
$$

Se $w_{n_{0}} \notin M$ segue que $w_{n_{0}} \in B\left(A, \delta_{n_{0}}\right) \backslash M \subset \overline{B(A, \widetilde{\delta})} \backslash M$. Por hipótese temos

$$
\psi\left(\widetilde{\pi}\left(w_{n_{0}}, t_{n_{0}}\right)\right) \leq \psi\left(w_{n_{0}}\right) \leq \frac{\mu}{2}
$$

e isto contradiz (4.9).

Se $w_{n_{0}} \in M$, então existe $0<\varepsilon_{n_{0}}<t_{n_{0}}$ tal que $\tilde{\pi}\left(w_{n_{0}}, \varepsilon_{n_{0}}\right)=\pi\left(w_{n_{0}}, \varepsilon_{n_{0}}\right) \in\left(B\left(A, \delta_{n_{0}}\right) \backslash M\right) \subset$ $(B(A, v) \backslash M)$. Segue de (4.10) a seguinte desigualdade

$$
\psi\left(\tilde{\pi}\left(w_{n_{0}}, \varepsilon_{n_{0}}\right)\right) \leq \frac{\mu}{2}
$$

Agora, pelo Corolário 4.1.2 e pela desigualdade (4.12) temos

$$
\psi\left(\widetilde{\pi}\left(w_{n_{0}}, t_{n_{0}}\right)\right) \leq \psi\left(\widetilde{\pi}\left(w_{n_{0}}, \varepsilon_{n_{0}}\right)\right) \leq \frac{\mu}{2}
$$

o que contradiz (4.9).

Portanto existe um $\bar{\delta}>0$ com $0<\bar{\delta}<\widetilde{\delta}$ tal que

$$
\widetilde{\pi}(B(A, \bar{\delta}),[0,+\infty)) \subset B(A, \widetilde{\delta})
$$

O próximo resultado apresenta condições necessárias e suficientes para que um conjunto fechado seja uniformemente $\widetilde{\pi}$-estável. 
Teorema 4.1.8 Sejam $(X, \pi ; M, I)$ um sistema semidinâmico impulsivo e $A$ um subconjunto fechado de $X$.

1. Se existe uma função $\psi: X \rightarrow \mathbb{R}_{+}$satisfazendo as seguintes propriedades:

a) $\psi$ é contínua em $X \backslash(M \backslash A)$;

b) Para todo $\varepsilon>0$ existe um $\delta>0$ tal que $\psi(x) \geq \delta$ sempre que $\rho(x, A) \geq \varepsilon$ e $x \notin M$;

c) Para todo $\varepsilon>0$ existe um $\delta>0$ tal que $\psi(x) \leq \varepsilon$ sempre que $\rho(x, A) \leq \delta$;

d) $\psi(\pi(x, t)) \leq \psi(x)$ se $x \in X \backslash M$ e $0 \leq t \leq \phi(x)$, e $\psi(I(x)) \leq \psi(x)$ para $x \in M$. Então $A$ é uniformemente $\widetilde{\pi}$-estável.

2. Reciprocamente, se $A$ é uniformemente $\tilde{\pi}$-estável então existe uma função $\psi: X \rightarrow \mathbb{R}_{+}$satisfazendo as condições $a), b), c$ ) e $d$ ) acima.

Demonstração: 1 . Segue da condição $d)$ e da Proposição 4.1 .1 que $\psi(\tilde{\pi}(x, t)) \leq \psi(x)$ para todo $t \geq 0$ e para todo $x \in X \backslash M$. Em particular, dado $\varepsilon>0$, temos que $\psi(\tilde{\pi}(x, t)) \leq \psi(x)$ para todo $t \geq 0$ e para todo $x \in \overline{B(A, \varepsilon)} \backslash M$. Pelo Lema 4.1.7, existe $\delta>0$ com $0<\delta<\varepsilon$ tal que $\tilde{\pi}(B(A, \delta)),[0,+\infty)) \subset B(A, \varepsilon)$. Portanto $A$ é uniformemente $\widetilde{\pi}$-estável.

2. A função $\psi: X \rightarrow \mathbb{R}_{+}$definida por

$$
\psi(x)= \begin{cases}\sup _{k \geq 0}\left(\sup _{0 \leq t \leq \phi\left(x_{k}^{+}\right)} \frac{\rho\left(\pi\left(x_{k}^{+}, t\right), A\right)}{1+\rho\left(\pi\left(x_{k}^{+}, t\right), A\right)}\right), & \text { se } x \in X \backslash M \\ \psi(I(x)), & \text { se } x \in M\end{cases}
$$

satifaz as condições $a), b), c)$ e $d$ ) do enunciado.

Corolário 4.1.9 Sejam $(X, \pi ; M, I)$ um sistema semidinâmico impulsivo e $A$ um subconjunto fechado de $X$ tal que $M \subset A$. Então $A$ é uniformemente $\tilde{\pi}$-estável se, e somente se, existe uma função $\psi: X \rightarrow \mathbb{R}_{+}$que satisfaz as seguintes propriedades:

a) $\psi$ é contínua em $X$;

b) Para todo $\varepsilon>0$ existe um $\delta>0$ tal que $\psi(x) \geq \delta$ sempre que $\rho(x, A) \geq \varepsilon$; 
c) Para todo $\varepsilon>0$ existe um $\delta>0$ tal que $\psi(x) \leq \varepsilon$ sempre que $\rho(x, A) \leq \delta$;

d) $\psi(\pi(x, t)) \leq \psi(x)$ se $x \in X \backslash M$ e $t \geq 0$, e $\psi(I(x)) \leq \psi(x)$ para $x \in M$.

Agora, apresentamos o resultado de estabilidade assintótica para conjuntos compactos.

Teorema 4.1.10 Sejam $(X, \pi ; M, I)$ um sistema semidinâmico impulsivo, $X$ localmente compacto e $A$ um subconjunto compacto de $X$.

1. Se existe uma função $\psi: X \rightarrow \mathbb{R}_{+}$com as seguintes propriedades:

a) $\psi$ é contínua em $X \backslash(M \backslash A)$;

b) Para todo $\varepsilon>0$ existe um $\delta>0$ tal que $\psi(x) \geq \delta$ sempre que $\rho(x, A) \geq \varepsilon$ e $x \notin M$;

c) Para todo $\varepsilon>0$ existe um $\delta>0$ tal que $\psi(x) \leq \varepsilon$ sempre que $\rho(x, A) \leq \delta$;

d) $\psi(\pi(x, t)) \leq \psi(x)$ se $x \in X \backslash M$ e $0 \leq t \leq \phi(x)$, e $\psi(I(x)) \leq \psi(x)$ para $x \in M$;

$e)$ Existe um $\delta>0$ tal que se $x \in B(A, \delta) \backslash A$, então $\psi(\tilde{\pi}(x, t)) \stackrel{t \rightarrow+\infty}{\longrightarrow} 0$.

Então $A$ é assintoticamente $\widetilde{\pi}$-estável.

2. Reciprocamente, se $A$ é assintoticamente $\widetilde{\pi}$-estável então existe uma função $\psi: X \rightarrow \mathbb{R}_{+}$ satisfazendo as condições $a), b), c), d)$ e $e$ ) acima.

Demonstração: 1 . Pelo Teorema 4.1 .8 o conjunto $A$ é uniformemente $\widetilde{\pi}$-estável e pelo Teorema 3.1 .13 o conjunto $A$ é orbitalmente $\widetilde{\pi}$-estável. Basta mostrarmos que $\widetilde{P}_{w}^{+}(A)$ é uma vizinhança de A. Pelo item $e$ ), existe $\delta>0$ tal que $\psi(\tilde{\pi}(x, t)) \stackrel{t \rightarrow+\infty}{\longrightarrow} 0$ para todo $x \in B(A, \delta) \backslash A$. Afirmamos que $B(A, \delta) \subset \widetilde{P}_{w}^{+}(A)$. De fato, como $A$ é compacto e uniformemente $\widetilde{\pi}$-estável segue que $A$ é $\tilde{\pi}$-invariante. Assim $A \subset \widetilde{P}_{w}^{+}(A)$. Precisamos mostrar que $B(A, \delta) \backslash A \subset \widetilde{P}_{w}^{+}(A)$. Suponhamos que isso não ocorra, então existe $x \in B(A, \delta) \backslash A$ tal que $x \notin \widetilde{P}_{w}^{+}(A)$. Logo existem uma vizinhança $U$ de $A$ e $\tau \in \mathbb{R}_{+}$tais que

$$
\tilde{\pi}(x,[\tau,+\infty)) \cap U=\emptyset .
$$

Como $A$ é compacto, podemos obter um $v>0$ tal que $\rho(\tilde{\pi}(x, t), A) \geq v$ para todo $t>\tau$. Como $\tilde{\pi}(x, t) \notin M$ para $t \geq \tau$, pois $I(M) \cap M=\emptyset$, segue pelo item $b$ ) que dado $v>0$ acima, existe $\eta>0$ tal que

$$
\psi(\tilde{\pi}(x, t)) \geq \eta
$$


para todo $t \geq \tau$. Mas isto contradiz a condição $e)$. Portanto $B(A, \delta) \subset \widetilde{P}_{w}^{+}(A)$ e segue que $A$ um $\tilde{\pi}$-atrator fraco. Portanto $A$ é assintoticamente $\widetilde{\pi}$-estável.

2. A função $\psi: X \rightarrow \mathbb{R}_{+}$definida por

$$
\psi(x)= \begin{cases}\sup _{k \geq 0}\left(\sup _{0 \leq t \leq \phi\left(x_{k}^{+}\right)} \frac{\rho\left(\pi\left(x_{k}^{+}, t\right), A\right)}{1+\rho\left(\pi\left(x_{k}^{+}, t\right), A\right)}\right) & \text { se } x \in X \backslash M, \\ \psi(I(x)) & \text { se } x \in M,\end{cases}
$$

satisfaz os itens $a), b), c), d)$ e $e$ ). Vamos verificar apenas o item $e$ ). Como $A$ é assintoticamente $\widetilde{\pi}$-estável, temos que $\widetilde{P}_{w}^{+}(A)=\widetilde{P}^{+}(A)$ é uma vizinhança de $A$. Então existe um $\delta>0$ tal que $B(A, \delta) \subset \widetilde{P}^{+}(A)$. Seja $x \in B(A, \delta) \backslash A$, então $x \in \widetilde{P}^{+}(A)$. Dado $\varepsilon>0$ existe $\tau \geq 0$ tal que $\tilde{\pi}(x,[\tau,+\infty)) \subset B(A, \varepsilon)$. Logo pela definição de $\psi$ temos $\psi(\widetilde{\pi}(x, t)) \stackrel{t \rightarrow+\infty}{\longrightarrow} 0$.

Corolário 4.1.11 Seja $(X, \pi ; M, I)$ um sistema semidinâmico impulsivo, $X$ localmente compacto e $A$ um subconjunto compacto de $X$ tal que $M \subset A$. Então $A$ é assintoticamente $\tilde{\pi}$-estável se, e somente se, existe uma função $\psi: X \rightarrow \mathbb{R}_{+}$com as seguintes propriedades:

a) $\psi$ é contínua em $X$;

b) Para todo $\varepsilon>0$ existe um $\delta>0$ tal que $\psi(x) \geq \delta$ sempre que $\rho(x, A) \geq \varepsilon$;

c) Para todo $\varepsilon>0$ existe um $\delta>0$ tal que $\psi(x) \leq \varepsilon$ sempre que $\rho(x, A) \leq \delta$;

d) $\psi(\pi(x, t)) \leq \psi(x)$ se $x \in X \backslash M$ e $t \geq 0$, e $\psi(I(x)) \leq \psi(x)$ para $x \in M$;

$e)$ Existe um $\delta>0$ tal que se $x \in B(A, \delta) \backslash A$, então $\psi(\widetilde{\pi}(x, t)) \stackrel{t \rightarrow+\infty}{\longrightarrow} 0$.

Apresentamos abaixo um exemplo para ilustrar os resultados acima. Notemos que, mesmo sem conhecer a solução do sistema, conseguimos informações importante sobre a estabilidade de certos conjuntos.

Exemplo 4.1.12 Seja $X=\mathbb{R}^{2} \times\{0,1\}$ e consideremos o sistema semidinâmico $(X, \pi)$ dado pelo sistema autonômo

$$
\left\{\begin{array}{l}
x^{\prime}=g(x) \\
y^{\prime}=h(y),
\end{array}\right.
$$


em $\mathbb{R}^{2} \times\{0\}$ e em $\mathbb{R}^{2} \times\{1\}$ independentemente, onde $g, h: \mathbb{R} \rightarrow \mathbb{R}$ são funções contínuas satisfazendo as condições $x g(x)<0$ para todo $x \in \mathbb{R}$ e $y h(y)<0$ para todo $y \in \mathbb{R}$.

Consideremos os conjuntos

$$
M_{0}=\left\{(x, y, z) \in \mathbb{R}^{3}: x^{2}+y^{2}=1 \text { e } z=0\right\}
$$

e

$$
M_{1}=\left\{(x, y, z) \in \mathbb{R}^{3}: x^{2}+y^{2}=\frac{1}{4} \quad \text { e } \quad z=1\right\},
$$

e definamos o conjunto impulsivo $M=M_{0} \cup M_{1}$. Agora, definamos a função impulso $I: M \rightarrow \mathbb{R}^{3}$ por

$$
I(x, y, 0)=(x, y, 1) \text { para }(x, y, 0) \in M_{0}
$$

e

$$
I(x, y, 1)=(x, y, 0) \text { para }(x, y, 1) \in M_{1} .
$$

Assim, consideremos o sistema impulsivo $(X, \pi ; I, M)$.

Dados $A_{0}=\left\{(x, y) \in \mathbb{R}^{2}: x^{2}+y^{2} \leq 1\right\} \times\{0\}$ e $A_{1}=\left\{(x, y) \in \mathbb{R}^{2}: x^{2}+y^{2} \leq 1\right\} \times\{1\}$, temos que $A=A_{0} \cup A_{1}$ é assintoticamente $\tilde{\pi}$-estável. De fato, definamos a função $\psi: X \rightarrow \mathbb{R}_{+}$por:

$$
\psi(x, y, z)= \begin{cases}\frac{\sqrt{x^{2}+y^{2}}-1}{\sqrt{x^{2}+y^{2}}}, & \text { se } \quad(x, y, z) \in X \backslash A \\ 0, & \text { se } \quad(x, y, z) \in A\end{cases}
$$

Como $M \subset A$, mostraremos que as condições do Corolário 4.1.11 são válidas.

a) É fácil ver que $\psi$ é contínua em $X$;

b) Dado $\varepsilon>0$, tomemos $\delta=\frac{\varepsilon}{\varepsilon+1}$. Se $\rho\left((x, y, 0), A_{0}\right) \geq \varepsilon$ então

$$
\psi(x, y, 0)=\frac{\sqrt{x^{2}+y^{2}}-1}{\sqrt{x^{2}+y^{2}}} \geq \frac{\varepsilon}{\varepsilon+1}=\delta .
$$

Analogamente, se $\rho\left((x, y, 1), A_{1}\right) \geq \varepsilon$ então $\psi(x, y, 1) \geq \delta$. 
c) Dado $\varepsilon>0, \varepsilon<1$, tomemos $\delta=\frac{\varepsilon}{1-\varepsilon}$. Assim, se $\rho\left((x, y, 0), A_{0}\right)<\delta$ temos:

- $\rho\left((x, y, 0), A_{0}\right)<\delta$ e $\sqrt{x^{2}+y^{2}} \leq 1$, então $\psi(x, y, 0)=0<\varepsilon$

- $\rho\left((x, y, 0), A_{0}\right)<\delta$ e $\sqrt{x^{2}+y^{2}}>1$, então

$$
\psi(x, y, 0)=\frac{\sqrt{x^{2}+y^{2}}-1}{\sqrt{x^{2}+y^{2}}}<\frac{\frac{\varepsilon}{1-\varepsilon}}{\frac{\varepsilon}{1-\varepsilon}+1}=\varepsilon .
$$

Analogamente, se $\rho\left((x, y, 1), A_{1}\right)<\delta$ segue que $\psi(x, y, 1)<\varepsilon$.

d) Sejam $x(t)=x\left(t ; 0, x_{0}\right)$ e $y(t)=y\left(t ; 0, y_{0}\right)$ as soluções dos sistemas

$$
\left\{\begin{array} { l } 
{ x ^ { \prime } = g ( x ) } \\
{ x ( 0 ) = x _ { 0 } }
\end{array} \quad \text { e } \quad \left\{\begin{array}{l}
y^{\prime}=h(y) \\
y(0)=y_{0}
\end{array}\right.\right.
$$

respectivamente. Sejam $z_{0}=\left(x_{0}, y_{0}, 0\right)$ e $w_{0}=\left(x_{0}, y_{0}, 1\right)$. Agora, consideremos os semifluxos $\varphi_{1}\left(z_{0}, t\right)=(x(t), y(t), 0)$ e $\varphi_{2}\left(w_{0}, t\right)=(x(t), y(t), 1)$.

Se $\sqrt{x_{0}^{2}+y_{0}^{2}}>1$, então

$$
\psi^{\prime}\left(\varphi_{1}\left(z_{0}, t\right)\right)=\frac{\partial \psi}{\partial x} \dot{x}(t)+\frac{\partial \psi}{\partial y} \dot{y}(t)=\frac{x(t) g(x(t))+y(t) h(y(t))}{\sqrt{\left(x^{2}(t)+y^{2}(t)\right)^{3}}}<0
$$

para todo $t \geq 0$ tal que $(x(t), y(t)) \notin A_{0}$. Se $(x(t), y(t)) \in A_{0}$ segue que $\psi^{\prime}\left(\varphi_{1}\left(z_{0}, t\right)\right)=0$. Logo,

$$
\psi^{\prime}\left(\varphi_{1}\left(z_{0}, t\right)\right) \leq 0
$$

para todo $t \geq 0$ e todo $\left(x_{0}, y_{0}\right) \in \mathbb{R}^{2}$. Portanto, $\psi\left(\varphi_{1}\left(z_{0}, t\right)\right) \leq \psi\left(z_{0}\right)$ para $t \geq 0$.

De mesmo modo, mostramos que $\psi\left(\varphi_{2}\left(w_{0}, t\right)\right) \leq \psi\left(w_{0}\right)$ para todo $t \geq 0$ e todo $w_{0} \in \mathbb{R}^{2} \times\{1\}$.

Como $M \subset A$ e $I(M) \subset A$, temos que $\psi(I(x, y, z))=0=\psi(x, y, z)$ para todo $(x, y, z) \in M$.

e) Vamos considerar as notações do item $d$ ). Pelo Corolário 4.1.9, o conjunto $A$ é uniformemente $\tilde{\pi}$-estável, pois $\psi$ satisfaz as quatros condições demonstradas acima. Logo, dado $\varepsilon>0$ existe 
$\delta>0$ tal que

$$
\widetilde{\varphi}_{1}(B(A, \delta),[0,+\infty)) \subset B(A, \varepsilon) \quad \text { e } \quad \widetilde{\varphi}_{2}(B(A, \delta),[0,+\infty)) \subset B(A, \varepsilon)
$$

Como $A$ é uniformemente $\widetilde{\pi}$-estável então $A$ é positivamente $\widetilde{\pi}$-invariante (veja Teorema 3.1.3). E como $M \subset A$, podemos fazer a análise deste item apenas para o sistema semidinâmico sem impulsos.

Se $z_{0} \in B\left(A_{0}, \delta\right) \backslash A_{0} . \quad$ Como $\psi^{\prime}\left(\varphi_{1}\left(z_{0}, t\right)\right) \leq 0$ para todo $t \geq 0$, segue que o limite $\lim _{t \rightarrow+\infty} \psi\left(\varphi_{1}\left(z_{0}, t\right)\right)$ existe. Suponhamos por contradição que

$$
\lim _{t \rightarrow+\infty} \psi\left(\varphi_{1}\left(z_{0}, t\right)\right)=\ell>0
$$

Defina

$$
K=\left\{(x, y, z) \in \mathbb{R}^{2} \times\{0\}: \rho\left((x, y, z), A_{0}\right) \leq \varepsilon \quad \text { e } \quad \psi(x, y, z) \geq \ell\right\}
$$

Notemos que $K$ é compacto. Assim podemos definir

$$
\eta:=\min \{-\dot{\psi}(w): w \in K\}
$$

Como $A$ não está contido em $K$, pois $\psi(A)=0$, segue que $\eta>0$. Então $-\dot{\psi}\left(\varphi_{1}\left(z_{0}, t\right)\right) \geq \eta$ para todo $t \geq 0$. Integrando a inequação de 0 a $t$, obtemos

$$
\psi\left(\varphi_{1}\left(z_{0}, t\right)\right) \leq \psi\left(z_{0}\right)-\eta t
$$

para todo $t \geq 0$. Mas isto é uma contradição já que $\psi$ é uma função positiva. Portanto

$$
\psi\left(\varphi_{1}\left(z_{0}, t\right)\right) \stackrel{t \rightarrow+\infty}{\longrightarrow} 0
$$

Analogamente, mostramos que se $w_{0} \in B\left(A_{1}, \delta\right) \backslash A_{1}$, então $\lim _{t \rightarrow+\infty} \psi\left(\varphi_{2}\left(w_{0}, t\right)\right)=0$.

Portanto, pelo Corolário 4.1.11, o conjunto $A$ é assintoticamente $\widetilde{\pi}$-estável. 


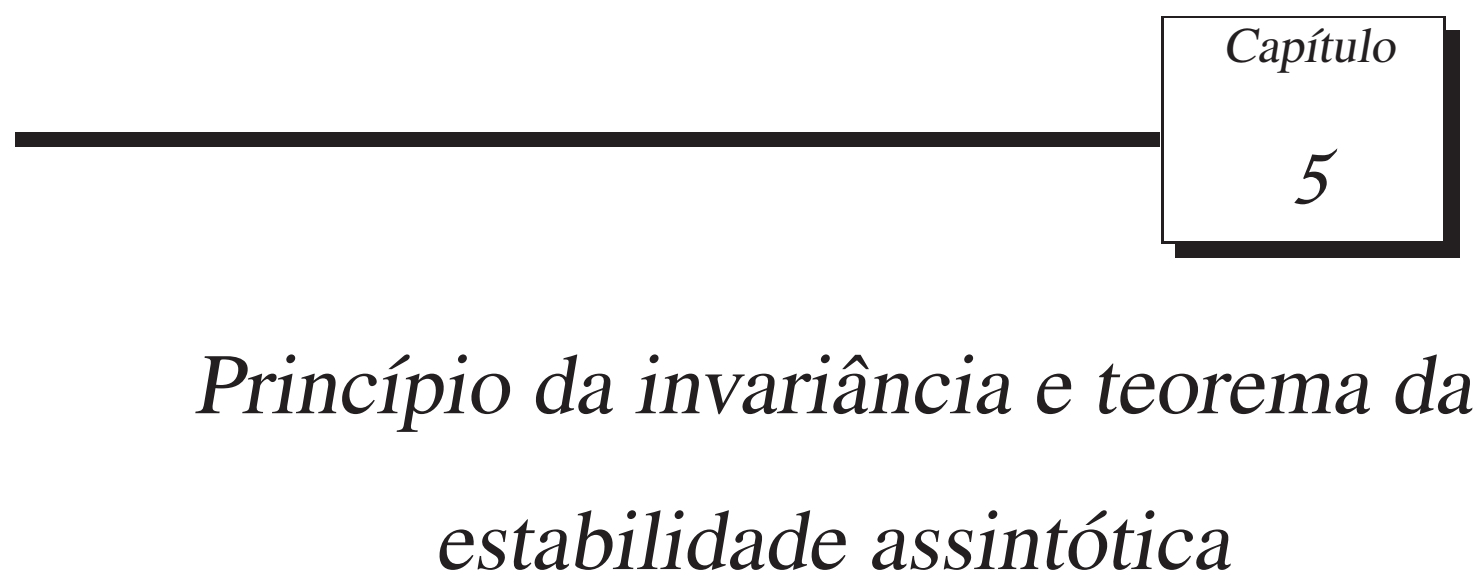

Neste capítulo, apresentamos a versão do Princípio da Invariância de LaSalle e a versão do Teorema da Estabilidade Assintótica para sistemas semidinâmicos com impulsos. As referências utilizadas foram [8] e [18].

\subsection{Princípio da invariância de LaSalle}

O Princípio da Invariância de LaSalle é uma ferramenta muito importante para o estudo do comportamento assintótico de soluções de equações diferenciais. Este princípio fornece um meio de estudar a estabilidade de um sistema sem o conhecimento das soluções das equações diferenciais. Para isto, utilizamos uma função auxiliar denominada função de Lyapunov.

Nesta seção apresentamos a versão do Princípio da Invariância de LaSalle para sistemas semidinâmicos impulsivos. Iniciamos com a definição de função de Lyapunov que iremos utilizar neste capítulo.

Definição 5.1.1 Uma função de Lyapunov em um sistema semidinâmico impulsivo $(X, \pi ; I, M)$ é uma função $V: X \rightarrow \mathbb{R}$ que satisfaz as seguintes condições: 

a) $V$ é contínua em $X$;
b) $V(I(x)) \leq V(x)$ para todo $x \in M$;
c) $V^{\prime}(x) \leq 0$ para todo $x \in X$, onde $V^{\prime}(x)=\lim _{t \rightarrow 0+} \frac{V(\tilde{\pi}(x, t))-V(x)}{t}$.

Segue da condição $c$ ) acima que $V(\pi(x, t)) \leq V(x)$ para todo $t \geq 0$ e para todo $x \in X$. Assim, pela Proposição 4.1.1, temos que $V(\tilde{\pi}(x, t)) \leq V(x)$ para todo $t \geq 0$ e para todo $x \in X$.

Dado um sistema semidinâmico impulsivo $(X, \pi ; M, I)$, definimos:

i) $G \subset X$ um subconjunto fechado e positivamente $\tilde{\pi}$-invariante;

ii) $E=\left\{x \in G: V^{\prime}(x)=0\right\}$;

iii) $A \subset E$ o maior subconjunto positivamente $\tilde{\pi}$-invariante contido em $E$.

Observemos que se $x \in A$, então $V^{\prime}(\tilde{\pi}(x, t))=0$ para todo $t \geq 0$.

A seguir, apresentamos a primeira versão do Princípio da Invariância de LaSalle para sistemas impulsivos.

Teorema 5.1.2 Sejam $(X, \pi ; M, I)$ um sistema semidinâmico impulsivo e $V$ uma função de Lyapunov. Suponhamos que $\widetilde{L}^{+}(x) \cap M=\emptyset$ para todo $x \in G$ e $I(M) \cap M=\emptyset$. Então para qualquer $x \in G$ existe um número real $\alpha$ tal que $\widetilde{L}^{+}(x) \subset A \cap V^{-1}(\alpha)$.

Demonstração: Seja $x \in G$ e suponhamos que $\widetilde{L}^{+}(x) \neq \emptyset$. Como $G$ é fechado e positivamente $\tilde{\pi}$-invariante, temos que $\widetilde{L}^{+}(x) \subset G$.

Primeiro, suponhamos que $\widetilde{L}^{+}(x)$ seja unitário. Então existe $p_{0} \in G$ tal que $\widetilde{L}^{+}(x)=\left\{p_{0}\right\}$. Como $\widetilde{L}^{+}(x) \cap M=\emptyset$, segue que $\widetilde{L}^{+}(x)$ é positivamente $\widetilde{\pi}$-invariante pelo Corolário 2.4.7. Logo,

$$
\tilde{\pi}\left(p_{0}, t\right)=p_{0},
$$

para todo $t \geq 0$. Assim, $V^{\prime}\left(p_{0}\right)=0$ já que $V\left(\tilde{\pi}\left(p_{0}, t\right)\right)=V\left(p_{0}\right)$ para todo $t \geq 0$. Portanto $\widetilde{L}^{+}(x) \subset E$ e como $A$ é o maior subconjunto positivamente $\tilde{\pi}$-invariante de $E$, segue que $\widetilde{L}^{+}(x) \subset A$. Definindo $V\left(p_{0}\right)=\alpha$, concluímos que $\widetilde{L}^{+}(x) \subset A \cap V^{-1}(\alpha)$. 
Agora, suponhamos que $\widetilde{L}^{+}(x)$ não seja unitário. Então existem $y_{1}, y_{2} \in \widetilde{L}^{+}(x)$ com $y_{1} \neq y_{2}$. Logo existem sequências de números reais positivos $\left\{t_{n}\right\}_{n \geq 1}$ e $\left\{s_{n}\right\}_{n \geq 1}$ tais que $t_{n} \stackrel{n \rightarrow+\infty}{\longrightarrow}+\infty, s_{n} \stackrel{n \rightarrow+\infty}{\longrightarrow}+\infty$,

$$
\tilde{\pi}\left(x, t_{n}\right) \stackrel{n \rightarrow+\infty}{\longrightarrow} y_{1} \quad \text { e } \quad \tilde{\pi}\left(x, s_{n}\right) \stackrel{n \rightarrow+\infty}{\longrightarrow} y_{2} .
$$

Como $t_{n} \stackrel{n \rightarrow+\infty}{\longrightarrow}+\infty$ e $s_{n} \stackrel{n \rightarrow+\infty}{\longrightarrow}+\infty$, podemos encontrar subsequências $\left\{t_{n_{k}}\right\}_{k \geq 1}$ e $\left\{s_{n_{k}}\right\}_{k \geq 1}$ tais que $t_{n_{k}}>s_{n_{k}}$ para todo $k \geq 1$. Pelo Corolário 4.1.2 temos

$$
V\left(\tilde{\pi}\left(x, t_{n_{k}}\right)\right) \leq V\left(\tilde{\pi}\left(x, s_{n_{k}}\right)\right)
$$

para todo $k \geq 1$. Passando ao limite, quando $k \rightarrow+\infty$, obtemos $V\left(y_{1}\right) \leq V\left(y_{2}\right)$.

Da mesma maneira, podemos encontrar subsequências $\left\{t_{n_{\ell}}\right\}_{\ell \geq 1}$ e $\left\{s_{n_{\ell}}\right\}_{\ell \geq 1}$ tais que $t_{n_{\ell}}<s_{n_{\ell}}$ para todo $\ell \geq 1$. Pelo Corolário 4.1.2 obtemos

$$
V\left(\tilde{\pi}\left(x, s_{n_{\ell}}\right)\right) \leq V\left(\tilde{\pi}\left(x, t_{n_{\ell}}\right)\right)
$$

para todo $\ell \geq 1$. Passando ao limite, quando $\ell \rightarrow+\infty$, obtemos $V\left(y_{2}\right) \leq V\left(y_{1}\right)$.

Portanto $V\left(y_{1}\right)=V\left(y_{2}\right)$. Consequentemente, $V(y)=\alpha$ para todo $y \in \widetilde{L}^{+}(x)$ e para algum $\alpha$ real. Logo $V^{\prime}(y)=0$ para todo $y \in \widetilde{L}^{+}(x)$ e portanto $\widetilde{L}^{+}(x) \subset E$. Segue da invariância positiva de $\widetilde{L}^{+}(x)$ (pois $\widetilde{L}^{+}(x) \cap M=\emptyset$ ) e do fato de $A$ ser o maior subconjunto positivamente $\widetilde{\pi}$-invariante de $E$ que $\widetilde{L}^{+}(x) \subset A \cap V^{-1}(\alpha)$. O resultado está provado.

Apresentamos, a seguir, o segundo Princípio da Invariância de LaSalle para sistemas impulsivos no caso em que $\widetilde{L}^{+}(x) \cap M \neq \emptyset$ para algum $x \in G$.

Teorema 5.1.3 Sejam $(X, \pi ; M, I)$ um sistema semidinâmico impulsivo e $V: X \rightarrow \mathbb{R}_{+}$uma função de Lyapunov tal que $V(x)=0$ para todo $x \in M$. Suponhamos que $I(M) \cap M=\emptyset$. Se para algum $x \in G, \widetilde{L}^{+}(x) \cap M \neq \emptyset$, então existe um número real $\alpha$ tal que $\widetilde{L}^{+}(x) \subset A \cap V^{-1}(\{0\} \cup\{\alpha\})$.

Demonstração: Suponhamos que $\widetilde{L}^{+}(x) \cap M \neq \emptyset$ para algum $x \in G$. Podemos escrever

$$
\widetilde{L}^{+}(x) \cap M=\left\{z_{k}\right\}_{k \in \Lambda}
$$


onde $\Lambda$ é um conjunto de índices. Temos que $\widetilde{L}^{+}(x) \subset G$ pois $G$ é fechado e positivamente $\tilde{\pi}$-invariante. Usando a Proposição 2.3.3 e o Teorema 2.4.6, temos que

$$
\Gamma_{x}:=\left(\widetilde{L}^{+}(x) \backslash M\right) \cup\left(\bigcup_{k \in \Lambda} \widetilde{\pi}^{+}\left(z_{k}\right)\right)
$$

é positivamente $\widetilde{\pi}$-invariante. Notemos, também, que $\Gamma_{x} \subset G$ pois $\left\{z_{k}\right\}_{k \in \Lambda} \subset G$ e $G$ é positivamente $\tilde{\pi}$-invariante.

Afirmação: $V^{\prime}(w)=0$ para todo $w \in \Gamma_{x}$ e existe $\alpha \in \mathbb{R}$ tal que $\Gamma_{x} \subset V^{-1}(\{0\} \cup\{\alpha\})$.

De fato, como $\left\{z_{k}\right\}_{k \in \Lambda} \subset M$ segue que $V\left(z_{k}\right)=0$ para todo $k \in \Lambda$. Assim, para cada $k \in \Lambda$ fixado, temos

$$
0 \leq V\left(\pi\left(z_{k}, t\right)\right) \leq V\left(z_{k}\right)=0 \quad \text { para todo } \quad t \geq 0
$$

Logo, $V^{\prime}\left(\pi\left(z_{k}, t\right)\right)=0$ para todo $t \geq 0$ e para todo $k \in \Lambda$. Portanto $V^{\prime}(w)=0$ para todo $w \in$ $\bigcup_{k \in \Lambda} \tilde{\pi}^{+}\left(z_{k}\right)$ e $\bigcup_{k \in \Lambda} \tilde{\pi}^{+}\left(z_{k}\right) \subset V^{-1}(0)$.

Como $\widetilde{L}^{+}(x) \backslash M$ é positivamente $\widetilde{\pi}$-invariante (pelo Teorema 2.4.6), segue da prova do Teorema 5.1.2 que existe um número real $\alpha$ tal que $V(w)=\alpha$ para todo $w \in \widetilde{L}^{+}(x) \backslash M$, isto é, $V^{\prime}(w)=0$ para todo $w \in \widetilde{L}^{+}(x) \backslash M$. Assim a afirmação está demonstrada e pela definição do conjunto $A$, podemos concluir que

$$
\widetilde{L}^{+}(x) \subset \Gamma_{x} \subset A \cap V^{-1}(\{0\} \cup\{\alpha\})
$$

Isto prova o teorema.

\subsection{Teorema da estabilidade assintótica}

Nesta seção apresentamos o Teorema da Estabilidade Assintótica para o caso impulsivo. Consideramos o conjunto $G$ compacto e com auxílio de uma função de Lyapunov, estabelecemos condições para que o maior subconjunto positivamente $\tilde{\pi}$-invariante $A$ de $E$ seja $\tilde{\pi}$-atrator e assintoticamente $\tilde{\pi}$-estável.

Teorema 5.2.1 Sejam $(X, \pi ; M, I)$ um sistema semidinâmico impulsivo e $V: X \rightarrow \mathbb{R}$ uma função de Lyapunov. Suponhamos que $X$ seja localmente compacto, $G$ seja compacto em $X, I(M) \cap M=\emptyset$ 
e $\widetilde{L}^{+}(x) \cap M=\emptyset$ para todo $x \in G$.

i) Se $A \subset \operatorname{int}(G)$, então $A$ é $\tilde{\pi}$-atrator.

ii) Se, além disso, $V^{-1}(\alpha)=A$ para algum $\alpha \in \mathbb{R}_{+}$, então $A$ é assintoticamente $\tilde{\pi}$-estável.

Demonstração: $i$ ) Devemos mostrar que $\widetilde{P}^{+}(A)$ é uma vizinhança de $A$. Afirmamos que $\operatorname{int}(G) \subset \widetilde{P}^{+}(A)$. Suponhamos que isso não ocorra, isto é, existe $x \in \operatorname{int}(G)$ tal que $x \notin \widetilde{P}^{+}(A)$. Segue da invariância de $G$ que $\widetilde{\pi}^{+}(x) \subset G$. Como $x \notin \widetilde{P}^{+}(A)$, existe um aberto $U$ tal que $A \subset U \subset \operatorname{int}(G)$ e existe uma sequência $\left\{\tau_{n}\right\}_{n \geq 1} \subset \mathbb{R}_{+}$tal que $\tau_{n} \stackrel{n \rightarrow+\infty}{\longrightarrow}+\infty$ e

$$
\tilde{\pi}\left(x, \tau_{n}\right) \in G \backslash U
$$

para todo $n \in \mathbb{N}$. Como $G \backslash U$ é um conjunto compacto podemos supor, sem perda de generalidade, que

$$
\tilde{\pi}\left(x, \tau_{n}\right) \stackrel{n \rightarrow+\infty}{\longrightarrow} y \in G \backslash U .
$$

Agora, notemos que $y \in \widetilde{L}^{+}(x)$, o que é um absurdo pois pelo Teorema 5.1.2 temos que $\widetilde{L}^{+}(x) \subset A$. Portanto $\operatorname{int}(G) \subset \widetilde{P}^{+}(A)$ e $A$ é $\widetilde{\pi}$-atrator.

ii) Notemos que $A$ é compacto em $X$ pois $V^{-1}(\alpha)=A$ é fechado em $G$ o qual é compacto. Para mostrarmos que $A$ é orbitalmente $\tilde{\pi}$-estável, basta mostrarmos que $\widetilde{D}^{+}(A)=A$, veja Teorema 3.1.10. Como $A \subset \widetilde{D}^{+}(A)$, mostremos a outra inclusão. Seja $y \in \widetilde{D}^{+}(A)$ então $y \in \widetilde{D}^{+}(x)$ para algum $x \in A$, ou seja, existem sequências $\left\{x_{n}\right\}_{n \geq 1} \subset X$ e $\left\{t_{n}\right\}_{n \geq 1} \subset \mathbb{R}_{+}$tais que $x_{n} \stackrel{n \rightarrow+\infty}{\longrightarrow} x$ e $\tilde{\pi}\left(x_{n}, t_{n}\right) \stackrel{n \rightarrow+\infty}{\longrightarrow} y$.

Como $V$ é uma função de Lyapunov temos que $V\left(\tilde{\pi}\left(x_{n}, t_{n}\right)\right) \leq V\left(x_{n}\right)$ para todo $n \in \mathbb{N}$. Então pela continuidade de $V$ obtemos

$$
V(y) \leq V(x)=\alpha
$$

Suponhamos que $y \notin A$. Temos dois casos a considerar: quando $V(y)=\alpha$ e quando $V(y)<\alpha$.

Primeiro, suponhamos que $V(y)=\alpha$. Como $V^{-1}(\alpha)=A$, segue que $y \in A$ e isto é uma contradição. 
Agora, consideremos o caso em que $V(y)<\alpha$. Seja $V(y)=\beta$. Como $y \notin A$, $A$ é compacto e $V$ é contínua, existem $\delta>0$ e $\eta>0$ tais que $B(y, \eta) \cap B(A, \delta)=\emptyset$,

$$
|V(z)-\alpha|<\frac{\alpha-\beta}{3} \quad \text { para todo } \quad z \in B(A, \delta)
$$

e

$$
|V(z)-\beta|<\frac{\alpha-\beta}{3} \quad \text { para todo } \quad w \in B(y, \eta)
$$

Como $\tilde{\pi}\left(x_{n}, t_{n}\right) \stackrel{n \rightarrow+\infty}{\longrightarrow} y$, existe um natural $n_{1}>0$ tal que

$$
\tilde{\pi}\left(x_{n}, t_{n}\right) \in B(y, \eta)
$$

para todo $n>n_{1}$. Por outro lado, segue do fato de $A$ ser $\tilde{\pi}$-atrator que existe um natural $n_{2}>n_{1}$ tal que $x_{n} \in \widetilde{P}^{+}(A)$ para $n>n_{2}$. Desta forma, existe $\tau^{n}>t_{n}>0$ tal que

$$
\tilde{\pi}\left(x_{n},\left[\tau^{n},+\infty\right)\right) \subset B(A, \delta)
$$

para todo $n>n_{2}$. Então

$$
\alpha-\frac{\alpha-\beta}{3}<V\left(\tilde{\pi}\left(x_{n}, \tau^{n}\right)\right) \leq V\left(\widetilde{\pi}\left(x_{n}, t_{n}\right)\right)<\beta+\frac{\alpha-\beta}{3}
$$

para $n>n_{2}$, o que é uma contradição.

Portanto $y \in A$ e $\widetilde{D}^{+}(A)=A$. Assim $A$ é orbitalmente $\widetilde{\pi}$-estável e pelo Corolário 3.3 .9 concluímos que $A$ é assintoticamente $\tilde{\pi}$-estável.

Agora, apresentamos a versão do Teorema da Estabilidade Assintótica no caso em que $\widetilde{L}^{+}(x) \cap M \neq \emptyset$ para todo $x \in G$.

Teorema 5.2.2 Sejam $(X, \pi ; M, I)$ um sistema semidinâmico impulsivo e $V: X \rightarrow \mathbb{R}_{+}$uma função de Lyapunov. Suponhamos que $X$ seja localmente compacto, $G$ seja compacto em $X, I(M) \cap M=\emptyset$, $V(y)=0$ para todo $y \in M$ e $\widetilde{L}^{+}(x) \cap M \neq \emptyset$ para todo $x \in G$.

i) Se $A \subset \operatorname{int}(G)$, então $A$ é $\tilde{\pi}$-atrator. 
ii) Se, além disso, $V^{-1}(\{\alpha\} \cup\{0\})=A$ para algum $\alpha \in \mathbb{R}_{+}$, então $A$ é assintoticamente $\tilde{\pi}$-estável.

Demonstração: $i$ ) A prova segue os passos da demonstração do item $i$ ) do Teorema 5.2.1, usando o Teorema 5.1.3 ao invés do Teorema 5.1.2 para concluirmos que $\widetilde{L}^{+}(x) \subset A$.

ii) Como $A$ é $\tilde{\pi}$-atrator devemos mostrar que $\widetilde{D}^{+}(A)=A$ para concluirmos o resultado. Seja $y \in$ $\widetilde{D}^{+}(A)$ então $y \in \widetilde{D}^{+}(x)$ para algum $x \in A$, ou seja, existem sequências $\left\{x_{n}\right\}_{n \geq 1} \subset X$ e $\left\{t_{n}\right\}_{n \geq 1} \subset \mathbb{R}_{+}$tais que $x_{n} \stackrel{n \rightarrow+\infty}{\longrightarrow} x$ e $\widetilde{\pi}\left(x_{n}, t_{n}\right) \stackrel{n \rightarrow+\infty}{\longrightarrow} y$.

Como $V$ é uma função de Lyapunov temos $V\left(\widetilde{\pi}\left(x_{n}, t_{n}\right)\right) \leq V\left(x_{n}\right)$ para todo $n \in \mathbb{N}$. Então pela continuidade de $V$ obtemos

$$
V(y) \leq V(x)
$$

Por hipótese, $V(x)=0$ ou $V(x)=\alpha$.

Se $V(x)=0$, segue do fato de $V$ ser não negativa que $V(y)=0$. Como $V^{-1}(\{0\} \cup\{\alpha\})=A$, segue que $y \in A$.

Se $V(x)=\alpha$, suponhamos por absurdo que $y \notin A$. Usando a prova do item $i i)$ do Teorema 5.2.1 obtemos uma contradição. Logo $y \in A$.

Assim $\widetilde{D}^{+}(A)=A$ e $A$ é orbitalmente $\widetilde{\pi}$-estável. Portanto $A$ é assintoticamente $\widetilde{\pi}$-estável pelo Corolário 3.3.9. 


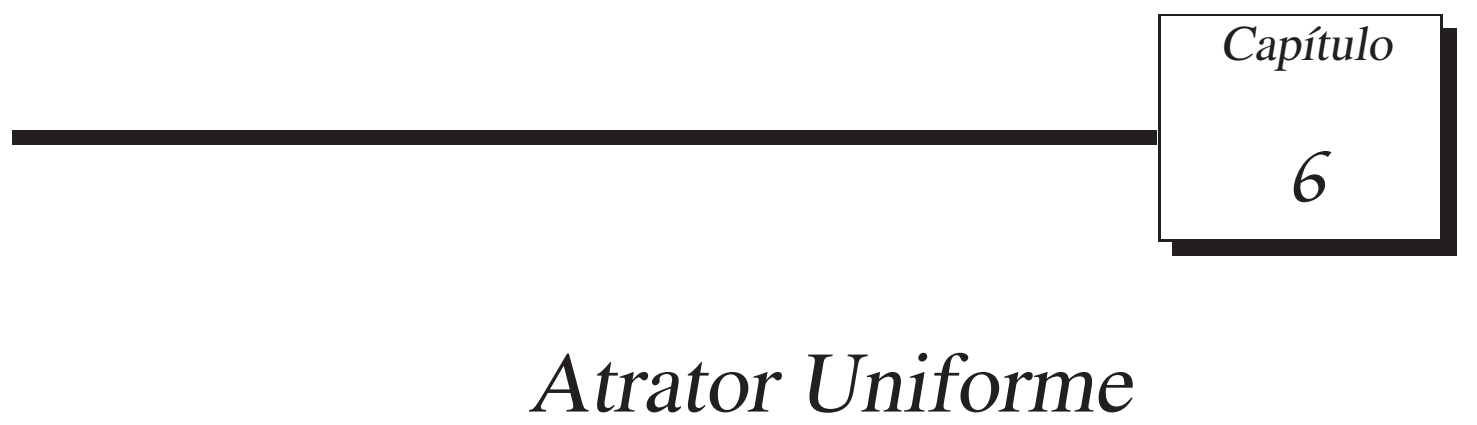

Neste capítulo, apresentamos nossa contribuição para esta dissertação. Estudamos os conceitos e resultados da região de atração uniforme para sistemas semidinâmicos contínuos e generalizamos estes resultados para os sistemas impulsivos. Os resultados deste capítulo estão apresentados no preprint [4].

\subsection{Região de atração uniforme}

Iniciamos com a definição da região de atração uniforme para um conjunto em um sistema semidinâmico impulsivo.

Definição 6.1.1 Sejam $(X, \pi ; M, I)$ um sistema semidinâmico impulsivo e $A$ um subconjunto de $X$. O conjunto

$\widetilde{P}_{u}^{+}(A)=\{x \in X:$ para toda vizinhança $U$ de $A$, existem uma vizinhança $V$ de $x$ e $T>0$

$$
\text { tais que } \tilde{\pi}(V, t) \subset U \text { para todo } t \geq T\}
$$

é chamado de região de atração uniforme de $A$ com respeito a $\widetilde{\pi}$. Se $x \in \widetilde{P}_{u}^{+}(A)$, dizemos que $x$ é uniformemente $\widetilde{\pi}$-atraído para $A$.

Definição 6.1.2 Dados $x \in X$ e $t \geq 0$ definimos $\widetilde{F}(x, t)=\{y \in X: \widetilde{\pi}(y, t)=x\}$. 
Considerando as regiões de atração e atração fraca definidas no Capítulo 3, Seção 3.3, podemos observar que

$$
\widetilde{P}_{u}^{+}(A) \subset \widetilde{P}^{+}(A) \subset \widetilde{P}_{w}^{+}(A),
$$

para todo subconjunto $A$ de $X$.

No caso contínuo, considerando um sistema semidinâmico $(X, \pi)$, temos que a região de atração uniforme de um conjunto é positivamente invariante quando $\pi$ possue a seguinte propriedade:

$$
\pi(G, t) \text { é um subconjunto aberto de } X
$$

para todo subconjunto aberto $G$ de $X$ e para todo $t \geq 0$. Como $\tilde{\pi}(G, t)$ pode ser um conjunto não aberto no caso impulsivo, mesmo $G$ sendo aberto, precisamos impor alguma outra condição para que a região de atração uniforme de um conjunto em $(X, \pi ; M, I)$ seja positivamente $\widetilde{\pi}$-invariante. A Proposição 6.1.4 estabelece condições para a invariância da região de atração uniforme. Antes de exibir o resultado, recordamos a definição do conceito da métrica de Hausdorff.

Definição 6.1.3 Seja $(X, \rho)$ um espaço métrico. Dados dois subconjuntos não vazios $A$ e $B$ de $X$, definimos a distância de Hausdorff entre $A$ e $B$ por

$$
\beta_{H}(A, B)=\max \left\{\sup _{x \in A} \rho(x, B), \sup _{y \in B} \rho(y, A)\right\} .
$$

Proposição 6.1.4 Sejam $(X, \pi ; M, I)$ um sistema semidinâmico impulsivo e $A$ um subconjunto de $X$. Suponhamos que para todo $x \in X$ e $t \geq 0$ tenhamos

$$
\beta_{H}\left(\widetilde{F}\left(x_{n}, t\right), \widetilde{F}(x, t)\right) \stackrel{n \rightarrow+\infty}{\longrightarrow} 0
$$

sempre que $\rho\left(x_{n}, x\right) \stackrel{n \rightarrow+\infty}{\longrightarrow} 0$. Então $\widetilde{P}_{u}^{+}(A)$ é positivamente $\widetilde{\pi}$-invariante.

Demonstração: Sejam $x \in \widetilde{P}_{u}^{+}(A)$ e $s \geq 0$. Dado uma vizinhança $U$ de $A$, existem uma vizinhança $V$ de $x$ e $T>0$ tais que

$$
\tilde{\pi}(V,[T,+\infty)) \subset U
$$

Consideremos o ponto $y=\tilde{\pi}(x, s)$ e seja $\widetilde{T}=\max \{0, T-s\}$. 
Afirmação: Existe um aberto $\mathscr{O}$ de $X$ tal que $y \in \mathscr{O}$ e $\tilde{\pi}(\mathscr{O}, \widetilde{T}) \subset \widetilde{\pi}(V,[T,+\infty))$.

De fato, suponhamos que isso não ocorra. Então existe uma sequência $\left\{w_{n}\right\}_{n \geq 1} \subset X$ tal que $w_{n} \stackrel{n \rightarrow+\infty}{\longrightarrow} y$ e $\tilde{\pi}\left(w_{n}, \widetilde{T}\right) \notin \tilde{\pi}(V,[T,+\infty))$ para todo $n \in \mathbb{N}^{*}$. Por hipótese, obtemos

$$
\beta_{H}\left(\widetilde{F}\left(w_{n}, s\right), \widetilde{F}(y, s)\right) \stackrel{n \rightarrow+\infty}{\longrightarrow} 0 .
$$

Como $x \in \widetilde{F}(y, s)$, segue que $\rho\left(x, \widetilde{F}\left(w_{n}, s\right)\right) \stackrel{n \rightarrow+\infty}{\longrightarrow} 0$. Logo existe uma sequência $\left\{z_{n}\right\}_{n \geq 1} \subset X$ com $z_{n} \in \widetilde{F}\left(w_{n}, s\right)$ tal que $z_{n} \stackrel{n \rightarrow+\infty}{\longrightarrow} x$. Portanto existe $n_{0} \in \mathbb{N}$ tal que $z_{n} \in V$ para todo $n \geq n_{0}$. Assim,

$$
\tilde{\pi}\left(w_{n}, \widetilde{T}\right)=\tilde{\pi}\left(z_{n}, s+\widetilde{T}\right) \in \tilde{\pi}(V,[T,+\infty)),
$$

para todo $n \geq n_{0}$. E isto é uma contradição. Logo existe um aberto $\mathscr{O}$ de $X$ tal que $y \in \mathscr{O}$ e $\tilde{\pi}(\mathscr{O}, \widetilde{T}) \subset \widetilde{\pi}(V,[T,+\infty))$. Assim

$$
\tilde{\pi}(\mathscr{O}, t) \subset U
$$

para todo $t \geq \widetilde{T}$. Portanto $y=\widetilde{\pi}(x, s) \in \widetilde{P}_{u}^{+}(A)$ e como $s$ é arbitrário temos que $\widetilde{P}_{u}^{+}(A)$ é positivamente $\tilde{\pi}$-invariante.

A próxima definição estabelece o conceito de atrator uniforme para um subconjunto $A$ em $(X, \pi ; M, I)$.

Definição 6.1.5 Sejam $(X, \pi ; M, I)$ um sistema semidinâmico impulsivo e $A$ um subconjunto de $X$. Dizemos que $A$ é um conjunto $\widetilde{\pi}$-atrator uniforme se $\widetilde{P}_{u}^{+}(A)$ é uma vizinhança de $A$.

Proposição 6.1.6 Sejam $(X, \pi ; M, I)$ um sistema semidinâmico impulsivo e $A$ um subconjunto de $X$. Suponhamos que $I(M) \cap M=\emptyset$. Se $A$ é $\widetilde{\pi}$-atrator uniforme então $\widetilde{P}_{u}^{+}(A)$ é positivamente $\widetilde{\pi}$-invariante e $\widetilde{P}_{u}^{+}(A) \backslash M$ é um conjunto aberto .

Demonstração: Primeiro, mostremos que $\widetilde{P}_{u}^{+}(A) \backslash M$ é um conjunto aberto. Seja $x \in \widetilde{P}_{u}^{+}(A) \backslash M$. Como $A$ é $\tilde{\pi}$-atrator uniforme, existem uma vizinhança $V$ de $x$ e $T>0$ tais que

$$
\widetilde{\pi}(V,[T,+\infty)) \subset \operatorname{int} \widetilde{P}_{u}^{+}(A)
$$

Podemos assumir que $V \cap M=\emptyset$. 
Afirmação: $V \subset \widetilde{P}_{u}^{+}(A) \backslash M$.

De fato, sejam $w \in V$ e $U$ uma vizinhança arbitrária de $A$. Como $\tilde{\pi}(w, T) \in \operatorname{int} \widetilde{P}_{u}^{+}(A)$, então existem um aberto $W$ e $s>0$ tais que $\tilde{\pi}(w, T) \in W \subset \operatorname{int} \widetilde{P}_{u}^{+}(A)$ e $\tilde{\pi}(W,[s,+\infty)) \subset U$. Temos dois casos a considerar: quando $T \neq \phi\left(x_{k}^{+}\right)$para todo $k=0,1,2 \ldots$, e quando $T=\phi\left(x_{k}^{+}\right)$para algum $k=1,2,3 \ldots$

Caso 1: Suponhamos que $T \neq \phi\left(x_{k}^{+}\right)$para todo $k=1,2,3 \ldots$. Como $w \notin M$, segue da continuidade de $\pi$ e $I$ que existe um aberto $\mathscr{O}$ de $X$ contendo $w$ tal que

$$
\tilde{\pi}(\mathscr{O}, T) \subset W
$$

Portanto $\tilde{\pi}(\mathscr{O},[T+s,+\infty)) \subset U$ e $w \in \widetilde{P}_{u}^{+}(A) \backslash M$.

Caso 2: Suponhamos que $T=\phi\left(x_{k}^{+}\right)$para algum $k \geq 0$. Então existe $\beta>0, \beta<\phi\left(x_{k+1}^{+}\right)$, tal que $\tilde{\pi}(w, T+\beta) \in W \backslash M$. Logo, podemos obter um aberto $\mathscr{U}$ de $X$ contendo $w$ tal que

$$
\tilde{\pi}(\mathscr{U}, T+\beta) \subset W .
$$

Portanto $\tilde{\pi}(\mathscr{U},[T+\beta+s,+\infty)) \subset U$ e $w \in \widetilde{P}_{u}^{+}(A) \backslash M$.

Portanto $x \in V \subset \widetilde{P}_{u}^{+}(A) \backslash M$ e $\widetilde{P}_{u}^{+}(A) \backslash M$ é aberto.

Mostremos, agora, que $\widetilde{P}_{u}^{+}(A)$ é positivamente $\widetilde{\pi}$-invariante. Sejam $x \in \widetilde{P}_{u}^{+}(A)$. Então existe $T>0$ tal que

$$
\widetilde{\pi}(x,[T,+\infty)) \subset \operatorname{int} \widetilde{P}_{u}^{+}(A) .
$$

Precisamos mostrar que $\tilde{\pi}(x,(0, T)) \subset \widetilde{P}_{u}^{+}(A)$. Com efeito, sejam $s \in(0, T)$ e $U$ uma vizinhança qualquer de $A$. Logo existem uma vizinhança $W$ de $\tilde{\pi}(x, T)$ e $r>0$ tais que $\tilde{\pi}(W,[r,+\infty)) \subset U$. Temos que considerar dois casos: quando $T \neq \phi\left(x_{k}^{+}\right)$para todo $k=1,2,3 \ldots$, e quando $T=\phi\left(x_{k}^{+}\right)$ para algum $k=1,2,3 \ldots$

Caso 1: Suponhamos que $T \neq \phi\left(x_{k}^{+}\right)$para todo $k=1,2,3 \ldots$ Como $\tilde{\pi}(x, s) \notin M$ pois $I(M) \cap M=\emptyset$, segue que existe um aberto $\mathscr{O}$ contendo $\tilde{\pi}(x, s)$ tal que

$$
\tilde{\pi}(\mathscr{O}, T-s) \subset W
$$


Portanto $\tilde{\pi}(\mathscr{O}, T+r-s) \subset U$ e $\tilde{\pi}(x, s) \in \widetilde{P}_{u}^{+}(A)$.

Caso 2: Suponhamos que $T=\phi\left(x_{k}^{+}\right)$para algum $k \geq 0$. Então existe um $\beta>0, \beta<\phi\left(x_{k+1}^{+}\right)$, tal que $\tilde{\pi}(w, T+\beta) \in W \backslash M$. Então existe um aberto $\mathscr{U}$ contendo $\tilde{\pi}(x, s)$ tal que

$$
\widetilde{\pi}(\mathscr{U}, T+\beta-s) \subset W .
$$

Portanto $\tilde{\pi}(\mathscr{U}, T+\beta+r-s) \subset U$ e $\tilde{\pi}(x, s) \in \widetilde{P}_{u}^{+}(A)$.

Desta forma, concluímos que $\widetilde{\pi}^{+}(x) \subset \widetilde{P}_{u}^{+}(A)$ e o resultado está provado.

Se $A$ é um subconjunto compacto de $X$, podemos dar uma caracterização para a região de atração $\widetilde{P}_{u}^{+}(A)$. Vejamos o próximo resultado.

Proposição 6.1.7 Sejam $(X, \pi ; M, I)$ um sistema semidinâmico impulsivo e $A$ um subconjunto compacto de $X$. Suponhamos $X$ localmente compacto. Então

$$
\widetilde{P}_{u}^{+}(A)=\left\{x \in X: \widetilde{J}^{+}(x) \neq \emptyset \text { e } \widetilde{J}^{+}(x) \subset A\right\}
$$

Demonstração: Mostremos a inclusão $\widetilde{P}_{u}^{+}(A) \subset\left\{x \in X: \widetilde{J}^{+}(x) \neq \emptyset\right.$ e $\left.\widetilde{J}^{+}(x) \subset A\right\}$. Sejam $y \in \widetilde{P}_{u}^{+}(A)$ e $U$ uma vizinhança de $A$ tal que $\bar{U}$ seja compacto. Então existem uma vizinhança $V$ de $y$ e $T>0$ tais que

$$
\tilde{\pi}(V,[T,+\infty)) \subset \bar{U}
$$

Afirmação: $\widetilde{L}^{+}(y) \neq \emptyset$.

Seja $\left\{t_{n}\right\}_{n \geq 1} \subset \mathbb{R}_{+}$uma sequência tal que $t_{n} \stackrel{n \rightarrow+\infty}{\longrightarrow}+\infty$. Então existe $n_{0} \in \mathbb{N}$ tal que

$$
\tilde{\pi}\left(y, t_{n}\right) \in \bar{U}
$$

para todo $n \geq n_{0}$. Pela compacidade de $\bar{U}$, podemos assumir sem perda de generalidade, que existe $a \in \bar{U}$ tal que

$$
\tilde{\pi}\left(y, t_{n}\right) \stackrel{n \rightarrow+\infty}{\longrightarrow} a .
$$

Assim $a \in \widetilde{L}^{+}(y)$ e a afirmação está provada. 
Como $\widetilde{L}^{+}(y) \subset \widetilde{J}^{+}(y)$, segue que $\widetilde{J}^{+}(y) \neq \emptyset$. Agora, notemos que

$$
\widetilde{J}^{+}(y) \subset \widetilde{\pi}(V,[T,+\infty)) \subset \bar{U}
$$

Como $U$ é arbitrário, segue que

$$
\widetilde{J}^{+}(y) \subset \cap\{\bar{U}: U \text { é vizinhança de } A\}=\bar{A}=A .
$$

Portanto $y \in\left\{x \in X: \widetilde{J}^{+}(x) \neq \emptyset\right.$ e $\left.\widetilde{J^{+}}(x) \subset A\right\}$.

Agora, mostremos a outra inclusão. Seja $x \in X$ tal que $\widetilde{J}^{+}(x) \neq \emptyset$ e $\widetilde{J}^{+}(x) \subset A$. Seja $U$ uma vizinhança de $A$. Como

$$
\widetilde{J}^{+}(x)=\bigcap_{\varepsilon>0} \bigcap_{t \geq 0} \overline{\bigcup \tilde{\pi}(B(x, \varepsilon), \tau): \tau \geq t\}},
$$

existem $\varepsilon_{0}>0$ e $t_{0}>0$ tais que

$$
\overline{\bigcup\left\{\tilde{\pi}\left(B\left(x, \varepsilon_{0}\right), \tau\right): \tau \geq t_{0}\right\}} \subset U
$$

Portanto, $\widetilde{\pi}\left(B\left(x, \varepsilon_{0}\right),\left[t_{0},+\infty\right)\right) \subset U$. Assim $x \in \widetilde{P}_{u}^{+}(A)$ e a demonstração está terminada.

O próximo resultado mostra algumas propriedades dos conjuntos $\widetilde{J}^{+}(x)$ e $\widetilde{D}^{+}(x), x \in X$. Lembremos que estamos supondo neste trabalho que $M$ satisfaz a condição STC e $M$ não contém pontos iniciais, veja Observação 2.2.12.

Teorema 6.1.8 Seja $(X, \pi ; M, I)$ um sistema semidinâmico impulsivo. Suponhamos que $X$ seja localmente compacto e que $I(M) \cap M=\emptyset$. Valem as propriedades:

i) Se $x \in X$, então $\overline{\widetilde{\pi}^{+}(x)} \subset \widetilde{D}^{+}(x)$.

ii) $\widetilde{D}^{+}(x)=\overline{\widetilde{\pi}^{+}(x)} \cup \widetilde{J}^{+}(x)$, para cada $x \in X \backslash M$.

iii) Se $x \in M$, então $\overline{\widetilde{\pi}^{+}(I(x))} \subset \widetilde{D}^{+}(x)$.

iv) $\widetilde{D}^{+}(x)=\overline{\widetilde{\pi}^{+}(x)} \cup \overline{\widetilde{\pi}^{+}(I(x))} \cup \widetilde{J}^{+}(x)$, para cada $x \in M$. 
Demonstração: $i)$ Seja $y \in \overline{\tilde{\pi}^{+}(x)}$, então existe uma sequência $\left\{t_{n}\right\}_{n \geq 1} \subset \mathbb{R}_{+}$tal que

$$
\tilde{\pi}\left(x, t_{n}\right) \stackrel{n \rightarrow+\infty}{\longrightarrow} y .
$$

Assim se consideramos a sequência $\left\{x_{n}\right\}_{n \geq 1} \subset X$ tal que $x_{n}=x$ para todo $n \in \mathbb{N}$, obtemos $y \in \widetilde{D}^{+}(x)$.

ii) Seja $x \notin M$. A inclusão $\overline{\widetilde{\pi}^{+}(x)} \cup \widetilde{J}^{+}(x) \subset \widetilde{D}^{+}(x)$ é óbvia. Por outro lado, seja $y \in \widetilde{D}^{+}(x)$. Então existem sequências $\left\{t_{n}\right\}_{n \geq 1} \subset \mathbb{R}_{+}$e $\left\{x_{n}\right\}_{n \geq 1} \subset X$ tais que $x_{n} \stackrel{n \rightarrow+\infty}{\longrightarrow} x$ e $\tilde{\pi}\left(x_{n}, t_{n}\right) \stackrel{n \rightarrow+\infty}{\longrightarrow} y$. Se $t_{n} \stackrel{n \rightarrow+\infty}{\longrightarrow}+\infty$, então $y \in \widetilde{J}^{+}(x)$. Do contrário, podemos assumir sem perda de generalidade, que existe $t \in \mathbb{R}_{+}$tal que $t_{n} \stackrel{n \rightarrow+\infty}{\longrightarrow} t$. Pela Observação 2.1.7, existe um $k \geq 1$ tal que

$$
\widetilde{\pi}(x, t)=\pi\left(x_{k-1}^{+}, t^{\prime}\right)
$$

onde $t=\sum_{i=0}^{k-1} \phi\left(x_{i-1}^{+}\right)+t^{\prime}$ e $0 \leq t^{\prime}<\phi\left(x_{k-1}^{+}\right)$.

Se $k=1$, então $\tilde{\pi}(x, t)=\pi(x, t)$ e $0 \leq t<\phi(x)$. Como $x_{n} \stackrel{n \rightarrow+\infty}{\longrightarrow} x$ e $t_{n} \stackrel{n \rightarrow+\infty}{\longrightarrow} t$, temos que $t_{n}<\phi\left(x_{n}\right)$ para $n$ suficientemente grande, e portanto

$$
\tilde{\pi}\left(x_{n}, t_{n}\right)=\pi\left(x_{n}, t_{n}\right) \stackrel{n \rightarrow+\infty}{\longrightarrow} \pi(x, t)=\tilde{\pi}(x, t)=y \in \overline{\tilde{\pi}^{+}(x)} .
$$

Vamos assumir que $k \geq 2$.

Caso 1: Suponhamos, a menos de subsequência, que $t_{n} \leq t$ para todo $n \in \mathbb{N}^{*}$.

Como $x_{n} \stackrel{n \rightarrow+\infty}{\longrightarrow} x$ e $x \notin M$, temos que

$$
\left(x_{n}\right)_{j}^{+} \stackrel{n \rightarrow+\infty}{\longrightarrow} x_{j}^{+} \quad \text { e } \quad \phi\left(\left(x_{n}\right)_{j}^{+}\right) \stackrel{n \rightarrow+\infty}{\longrightarrow} \phi\left(x_{j}^{+}\right)
$$

para todo $j=0,1,2, \ldots$ Se $0<t^{\prime}<\phi\left(x_{k-1}^{+}\right)$, podemos escrever

$$
t_{n}=\sum_{i=0}^{k-1} \phi\left(\left(x_{n}\right)_{i-1}^{+}\right)+t_{n}^{\prime}
$$


$\operatorname{com} t_{n}^{\prime} \stackrel{n \rightarrow+\infty}{\longrightarrow} t^{\prime}$ e $0<t_{n}^{\prime}<\phi\left(\left(x_{n}\right)_{k-1}^{+}\right)$para $n$ suficientemente grande. Daí

$$
\tilde{\pi}\left(x_{n}, t_{n}\right)=\pi\left(\left(x_{n}\right)_{k-1}^{+}, t_{n}^{\prime}\right) \stackrel{n \rightarrow+\infty}{\longrightarrow} \pi\left(x_{k-1}^{+}, t^{\prime}\right)=\tilde{\pi}(x, t)=y \in \overline{\tilde{\pi}^{+}(x)} .
$$

Agora, se $t^{\prime}=0$. Então o termo $t_{n}$ é escrito da seguinte forma

$$
t_{n}=\sum_{i=0}^{k-2} \phi\left(\left(x_{n}\right)_{i-1}^{+}\right)+t_{n}^{\prime \prime}
$$

onde $0<t_{n}^{\prime \prime}<\phi\left(x_{k-2}^{+}\right)$e $t_{n}^{\prime} \stackrel{n \rightarrow+\infty}{\longrightarrow} \phi\left(x_{k-2}^{+}\right), n \in \mathbb{N}^{*}$. Assim

$$
\tilde{\pi}\left(x_{n}, t_{n}\right)=\pi\left(\left(x_{n}\right)_{k-2}^{+}, t_{n}^{\prime \prime}\right) \stackrel{n \rightarrow+\infty}{\longrightarrow} \pi\left(x_{k-2}^{+}, \phi\left(x_{k-2}^{+}\right)\right)=x_{k-1}=y \in \overline{\widetilde{\pi}^{+}(x)} .
$$

Caso 2: Suponhamos, a menos de subsequência, que $t_{n} \geq t$ para todo $n \in \mathbb{N}^{*}$.

Neste caso, escrevemos $t_{n}=\sum_{i=0}^{k-1} \phi\left(\left(x_{n}\right)_{i-1}^{+}\right)+t_{n}^{\prime} \operatorname{com} t_{n}^{\prime} \stackrel{n \rightarrow+\infty}{\longrightarrow} t^{\prime}$ e $0<t_{n}^{\prime}<\phi\left(\left(x_{n}\right)_{k-1}^{+}\right)$. Logo

$$
\tilde{\pi}\left(x_{n}, t_{n}\right)=\pi\left(\left(x_{n}\right)_{k-1}^{+}, t_{n}^{\prime}\right) \stackrel{n \rightarrow+\infty}{\longrightarrow} \pi\left(x_{k-1}^{+}, t^{\prime}\right)=\tilde{\pi}(x, t)=y \in \overline{\tilde{\pi}^{+}(x)} .
$$

Portanto $y \in \overline{\widetilde{\pi}^{+}(x)}$ e $\widetilde{D}^{+}(x) \subset \overline{\widetilde{\pi}^{+}(x)} \cup \widetilde{J}^{+}(x)$.

iii) Dado $x \in M$, existem $y \in X$ e $t>0$ tais que $\pi(y, t)=x$. Seja $\left\{\lambda_{n}\right\}_{\geq} \subset \mathbb{R}_{+}$uma sequência crescente tal que $\pi\left(y, \lambda_{n}\right) \stackrel{n \rightarrow+\infty}{\longrightarrow} x$. Defina $w_{n}=\pi\left(y, \lambda_{n}\right), n \in \mathbb{N}^{*}$. Então

$$
\tilde{\pi}\left(w_{n}, \phi\left(w_{n}\right)\right) \stackrel{n \rightarrow+\infty}{\longrightarrow} I(x)
$$

Note que $I(x) \notin M$, pois $I(M) \cap M=\emptyset$. Dado $s \geq 0$, segue do Lema 2.4.5 que existe uma sequência $\left\{\varepsilon_{n}\right\}_{n \geq 1} \subset \mathbb{R}$ tal que $\varepsilon_{n} \stackrel{n \rightarrow+\infty}{\longrightarrow} 0 \mathrm{e}$

$$
\tilde{\pi}\left(w_{n}, \phi\left(w_{n}\right)+\varepsilon_{n}+s\right) \stackrel{n \rightarrow+\infty}{\longrightarrow} \tilde{\pi}(I(x), s) .
$$

Logo $\widetilde{\pi}(I(x), s) \in \widetilde{D}^{+}(x)$ e como $s$ é arbitrário segue o resultado. 
iv) Seja $x \in M$. Pelos itens $i)$ e $i i i)$ temos a inclusão $\overline{\widetilde{\pi}^{+}(x)} \cup \overline{\widetilde{\pi}^{+}(I(x))} \cup \widetilde{J^{+}}(x) \subset \widetilde{D}^{+}(x)$. Por outro lado, seja $y \in \widetilde{D}^{+}(x)$. Então existem sequências $\left\{t_{n}\right\}_{n \geq 1} \subset \mathbb{R}_{+}$e $\left\{x_{n}\right\}_{n \geq 1} \subset X$ tais que $x_{n} \stackrel{n \rightarrow+\infty}{\longrightarrow} x$ e $\tilde{\pi}\left(x_{n}, t_{n}\right) \stackrel{n \rightarrow+\infty}{\longrightarrow} y$. Se $t_{n} \stackrel{n \rightarrow+\infty}{\longrightarrow}+\infty$, então $y \in \widetilde{J}^{+}(x)$. Do contrário, podemos assumir sem perda de generalidade, que existe $t \in \mathbb{R}_{+}$tal que $t_{n} \stackrel{n \rightarrow+\infty}{\longrightarrow} t$. Pela Observação 2.1.7, existe um $k \geq 1$ tal que

$$
\tilde{\pi}(I(x), t)=\pi\left((I(x))_{k-1}^{+}, t^{\prime}\right)
$$

onde $t=\sum_{i=0}^{k-1} \phi\left((I(x))_{i-1}^{+}\right)+t^{\prime}$ e $0 \leq t^{\prime}<\phi\left((I(x))_{k-1}^{+}\right)$.

Como $x$ satisfaz a condição STC, existe tubo $F(L,[0,2 \lambda])$ através de $x$ dado pela seção $S$. Como o tubo é uma vizinhança de $x$, existe um $\eta>0$ tal que

$$
B(x, \eta) \subset F(L,[0,2 \lambda])
$$

Denotemos $H_{1}$ e $H_{2}$ por

$$
H_{1}=F(L,(\lambda, 2 \lambda]) \cap B(x, \eta) \quad \text { e } \quad H_{2}=F(L,[0, \lambda]) \cap B(x, \eta) .
$$

Suponhamos, a menos de subsequência, que $\left\{x_{n}\right\}_{n \geq 1} \subset H_{1}$ e consideremos os casos seguintes.

Caso 1: Suponhamos, sem perda de generalidade, que $t_{n} \leq t$ para todo $n \in \mathbb{N}^{*}$.

Neste caso $\phi\left(x_{n}\right) \stackrel{n \rightarrow+\infty}{\longrightarrow} 0$ e $\pi\left(x_{n}, \phi\left(x_{n}\right)\right) \stackrel{n \rightarrow+\infty}{\longrightarrow} x$. Agora, notemos que

$$
\phi\left(\left(x_{n}\right)_{j+1}^{+}\right) \stackrel{n \rightarrow+\infty}{\longrightarrow} \phi\left((I(x))_{j}^{+}\right)
$$

para todo $j=0,1,2, \ldots$. Vamos considerar os casos quando $0<t^{\prime}<\phi\left((I(x))_{k-1}^{+}\right)$e quando $t^{\prime}=0$.

a) Suponhamos que $0<t^{\prime}<\phi\left((I(x))_{k-1}^{+}\right)$. Então podemos escrever

$$
t_{n}=\sum_{i=0}^{k} \phi\left(\left(x_{n}\right)_{i-1}^{+}\right)+t_{n}^{\prime}
$$

$\operatorname{com} t_{n}^{\prime} \stackrel{n \rightarrow+\infty}{\longrightarrow} t^{\prime}$ e $0<t_{n}^{\prime}<\phi\left(\left(x_{n}\right)_{k}^{+}\right)$para $n$ suficientemente grande. Daí 


$$
\tilde{\pi}\left(x_{n}, t_{n}\right)=\pi\left(\left(x_{n}\right)_{k}^{+}, t_{n}^{\prime}\right) \stackrel{n \rightarrow+\infty}{\longrightarrow} \pi\left((I(x))_{k-1}^{+}, t^{\prime}\right)=\tilde{\pi}(I(x), t)=y \in \overline{\tilde{\pi}^{+}(I(x))} .
$$

b) Suponhamos que $t^{\prime}=0$. Notemos que se $k=1$ então $t=0$ e $t_{n}=0$ para todo $n \in \mathbb{N}^{*}, \operatorname{logo}$

$$
\tilde{\pi}\left(x_{n}, t_{n}\right)=\tilde{\pi}\left(x_{n}, 0\right)=x_{n} \stackrel{n \rightarrow+\infty}{\longrightarrow} x=y \in \overline{\tilde{\pi}^{+}(x)} .
$$

Então seja $k \geq 2$. O termo $t_{n}$ é escrito da seguinte forma

$$
t_{n}=\sum_{i=0}^{k-1} \phi\left(\left(x_{n}\right)_{i-1}^{+}\right)+t_{n}^{\prime \prime}
$$

onde $0<t_{n}^{\prime \prime}<\phi\left(\left(x_{n}\right)_{k-1}^{+}\right)$e $t_{n}^{\prime \prime} \stackrel{n \rightarrow+\infty}{\longrightarrow} \phi\left((I(x))_{k-2}^{+}\right), n \in \mathbb{N}^{*}$. Assim

$$
\tilde{\pi}\left(x_{n}, t_{n}\right)=\pi\left(\left(x_{n}\right)_{k-1}^{+}, t_{n}^{\prime \prime}\right) \stackrel{n \rightarrow+\infty}{\longrightarrow} \pi\left((I(x))_{k-2}^{+}, \phi\left((I(x))_{k-2}^{+}\right)\right)=(I(x))_{k-1}=y \in \overline{\tilde{\pi}^{+}(I(x))} .
$$

Caso 2: Suponhamos, a menos de subsequência, que $t_{n} \geq t$ para todo $n \in \mathbb{N}^{*}$.

Neste caso, escrevemos $t_{n}=\sum_{i=0}^{k} \phi\left(\left(x_{n}\right)_{i-1}^{+}\right)+t_{n}^{\prime} \operatorname{com} t_{n}^{\prime} \stackrel{n \rightarrow+\infty}{\longrightarrow} t^{\prime}$ e $0<t_{n}^{\prime}<\phi\left(\left(x_{n}\right)_{k}^{+}\right)$para $n$ suficientemente grande. Logo

$$
\tilde{\pi}\left(x_{n}, t_{n}\right)=\pi\left(\left(x_{n}\right)_{k}^{+}, t_{n}^{\prime}\right) \stackrel{n \rightarrow+\infty}{\longrightarrow} \pi\left((I(x))_{k-1}^{+}, t^{\prime}\right)=\tilde{\pi}(I(x), t)=y \in \overline{\widetilde{\pi}^{+}(I(x))} .
$$

Por outro lado, vamos considerar o caso em que $\left\{x_{n}\right\}_{n \geq 1} \subset H_{2}$. Neste caso, temos que $\phi\left(\left(x_{n}\right)_{j}^{+}\right) \stackrel{n \rightarrow+\infty}{\longrightarrow} \phi\left(x_{j}^{+}\right)$, para todo $j=0,1,2, \ldots$ Assim, podemos usar a mesma prova do item ii) e concluir que $y \in \overline{\widetilde{\pi}^{+}(x)}$.

Portanto $\widetilde{D}^{+}(x)=\overline{\widetilde{\pi}^{+}(x)} \cup \overline{\widetilde{\pi}^{+}(I(x))} \cup \widetilde{J}^{+}(x)$ e o resultado está provado.

O seguinte resultado estabelece condições para que um conjunto compacto $\widetilde{\pi}$-atrator uniforme seja assintoticamente $\widetilde{\pi}$-estável.

Proposição 6.1.9 Sejam $(X, \pi ; M, I)$ um sistema semidinâmico impulsivo e $A$ um subconjunto compacto de $X$. Suponhamos que $X$ seja localmente compacto e $I(M) \cap M=\emptyset$. Se $A$ é positi- 
vamente $\tilde{\pi}$-invariante e $\tilde{\pi}$-atrator uniforme então $A$ é $\tilde{\pi}$-estável. E consequentemente $A$ é assintoticamente $\widetilde{\pi}$-estável.

Demonstração: Pelo Teorema 3.1.10, basta mostrarmos que $\widetilde{D}^{+}(A)=A$. Seja $x \in \widetilde{D}^{+}(A)$. Então existe $a \in A$ tal que $x \in \widetilde{D}^{+}(a)$. Como $A$ é positivamente $\widetilde{\pi}$-invariante e fechado, segue que $\overline{\tilde{\pi}^{+}(a)} \subset A$. Como $A$ é um $\tilde{\pi}$-atrator uniforme temos que $A \subset \widetilde{P}_{u}^{+}(A)$. Logo, pela Proposição 6.1.7, temos que $\widetilde{J}^{+}(a) \subset A$.

Caso 1: $a \notin M$.

Segue do item $\mathrm{ii}$ ) do Teorema 6.1.8 que

$$
\widetilde{D}^{+}(a)=\overline{\widetilde{\pi}^{+}(a)} \cup \widetilde{J}^{+}(a) \subset A
$$

$\operatorname{Assim} x \in A$.

Caso 2: $a \in M$.

Como $A$ é positivamente $\tilde{\pi}$-invariante e fechado, segue que $I(a) \in A$ e portanto $\overline{\widetilde{\pi}^{+}(I(a))} \subset A$. Assim, pelo Teorema 6.1.8 item iv), obtemos

$$
\widetilde{D}^{+}(a)=\overline{\widetilde{\pi}^{+}(a)} \cup \overline{\widetilde{\pi}^{+}(I(a))} \cup \widetilde{J}^{+}(a) \subset A .
$$

$\operatorname{Assim} x \in A$.

Portanto $\widetilde{D}^{+}(A)=A$ e $A$ é $\widetilde{\pi}$-estável.

A seguir, apresentamos um resultado auxiliar cuja demonstração encontra-se em [11].

Lema 6.1.10 Seja $(X, \pi ; M, I)$ um sistema semidinâmico impulsivo tal que $I(M) \cap M=\emptyset$. Seja $x \notin M$ e $y \in \widetilde{L}^{+}(x)$. Então $\widetilde{J}^{+}(x) \subset \widetilde{J}^{+}(y)$.

Proposição 6.1.11 Sejam $(X, \pi ; M, I)$ um sistema semidinâmico impulsivo e $A$ um subconjunto compacto de $X$ tal que $A \cap M=\emptyset$. Suponhamos que $X$ seja localmente compacto e $I(M) \cap M=\emptyset$. Se $A$ é assintoticamente $\tilde{\pi}$-estável então $A$ é $\tilde{\pi}$-atrator uniforme. 
Demonstração: Como $A$ é assintoticamente $\widetilde{\pi}$-estável temos que $A$ é um $\widetilde{\pi}$-atrator, veja Corolário 3.3.9. Assim, $\widetilde{P}^{+}(A)$ é uma vizinhança para $A$, ou seja, existe um aberto $V$ tal que $A \subset V \subset \widetilde{P}^{+}(A)$ e $V \cap M=\emptyset$.

Afirmação: $\widetilde{P}^{+}(A) \backslash M \subset \widetilde{P}_{u}^{+}(A) \backslash M$.

Seja $x \in P^{+}(A) \backslash M$ e $U$ uma vizinhança de $A$ tal que $\bar{U}$ é compacto. Então existe $\tau \geq 0$ tal que

$$
\tilde{\pi}(x,[\tau,+\infty)) \subset \bar{U}
$$

Portanto $\widetilde{L}^{+}(x) \neq \emptyset$ e $\widetilde{L}^{+}(x) \subset A$. Logo $\widetilde{J}^{+}(x) \neq \emptyset$.

Seja $a \in \widetilde{L}^{+}(x)$. Como $x \notin M$, segue do Lema 6.1.10 e da estabilidade de $A$ as seguintes desigualdades

$$
\widetilde{J}^{+}(x) \subset \widetilde{J}^{+}(a) \subset \widetilde{D}^{+}(a) \subset A .
$$

Então, pela Proposição 6.1.7, temos $x \in \widetilde{P}_{u}^{+}(A)$. Como $x \notin M$, segue que $\widetilde{P}^{+}(A) \backslash M \subset \widetilde{P}_{u}^{+}(A) \backslash M$. Assim, $A \subset V \subset \widetilde{P}^{+}(A) \backslash M \subset \widetilde{P}_{u}^{+}(A) \backslash M \subset \widetilde{P}_{u}^{+}(A)$. Portanto $A$ é $\widetilde{\pi}$-atrator uniforme. 


\section{Referências Bibliográficas}

[1] N. P. Bhatia and O. Hajek, Local semi-dynamical systems, Lecture Notes in Mathematics 90, Springer-Verlag, 1970.

[2] N. P. Bhatia and G. P. Szegö, Stability theory of dynamical systems, Grundlehren Math. Wiss., Band 161, Springer-Verlag, New York, 1970; reprint of the 1970 original in: Classics Math., Springer-Verlag, Berlin, 2002.

[3] E. M. Bonotto, Sistemas Semidinâmicos Impulsivos. Dissertação de Mestrado. Universidade de São Paulo, 2005.

[4] E. M. Bonotto and J. C. Ferreira, Uniform attractors in impulsive semidynamical systems. Preprint.

[5] E. M. Bonotto and M. Federson, Topological conjugation and asymptotic stability in impulsive semidynamical systems. J. Math. Anal. Appl. 326, (2007), 869-881.

[6] E. M. Bonotto, Flows of characteristic $0^{+}$in impulsive semidynamical systems, J. Math. Anal. Appl., 332 (1), (2007), 81-96.

[7] E. M. Bonotto and M. Federson, Limit sets and the Poincaré-Bendixson Theorem in semidynamical impulsive systems. J. Diff. Equations. 244 (2008), 2334-2349.

[8] E. M. Bonotto, LaSalle's Theorems in impulsive semidynamical systems. Nonlinear Analysis: Theory, Methods \& Applications. 71 (5-6), (2009), 2291-2297.

[9] E. M. Bonotto and M. Federson, Poisson stability for impulsive semidynamical systems. Nonlinear Analysis: Theory, Methods \& Applications, 71 (12), (2009), 6148-6156. 
[10] E. M. Bonotto and N. G. Grulha Jr., Lyapunov stability of closed sets in impulsive semidynamical systems. Eletronic Journal of Differential Equations, 78, (2010), 1-18.

[11] E. M. Bonotto and D. P. Demuner. Autonomous dissipative semidynamical systems with impulses. Cadernos de Matemática, 12 (1), (2011), 167-204.

[12] K. Ciesielski, Sections in semidynamical systems, Bull. Polish Acad. Sci. Math., 40, (1992), 297-307.

[13] K. Ciesielski, On semicontinuity in impulsive dynamical systems, Bull. Polish Acad. Sci. Math., 52, (2004), 71-80.

[14] K. Ciesielski, On stability in impulsive dynamical systems, Bull. Polish Acad. Sci. Math., 52, (2004), 81-91.

[15] S. K. Kaul, On impulsive semidynamical systems, J. Math. Anal. Appl., 150, (1990), 120-128.

[16] S. K. Kaul, On impulsive semidynamical systems II, Recursive properties. Nonlinear Anal., 16, (1991), 635-645.

[17] S. K. Kaul, On impulsive semidynamical systems III, Lyapunov stability. Recent Trends in Differential Equations, 335-345, World Sci. Ser. Appl. Anal., 1. Publishing, River Edge, NJ, 1992.

[18] S. K. Kaul, Stability and asymptotic stability in impulsive semidynamical systems. J. Appl. Math. Stochastic Anal., 7(4), (1994), 509-523.

[19] V. Lakshmikanthan, D. D. Bainov, P. S. Simeonov, Theory of Impulsive Differential Equations, Modern Applied Math., 6, World Scientific, 1989. 